\title{
FELIPE PARRA DO NASCIMENTO
}

A condição socioeconômica altera os efeitos da poluição do ar na saúde?

Tese apresentada à Faculdade de Medicina da Universidade de São Paulo para obtenção do título de Doutor em Ciências

Programa de Saúde Coletiva

Orientador: Prof. Dr. Nelson da Cruz Gouveia

\section{SÃO PAULO}

2020 



\section{FELIPE PARRA DO NASCIMENTO}

A condição socioeconômica altera os efeitos da poluição do ar na saúde?

Tese apresentada à Faculdade de Medicina da Universidade de São Paulo para obtenção do título de Doutor em Ciências

Programa de Saúde Coletiva

Orientador: Prof. Dr. Nelson da Cruz Gouveia

\section{SÃO PAULO}

2020 


\section{Dados Internacionais de Catalogação na Publicação (CIP)}

Preparada pela Biblioteca da

Faculdade de Medicina da Universidade de Săo Paulo

(Creproduçăo autorizada pelo autor

Nascimento, Felipe Parra do

A condiçào socioeconômica altera os efeitos da

poluição do ar na saúde? / Felipe Parra do

Nascimento. -- São Paulo, 2020.

Tese (doutorado)--Faculdade de Medicina da

Universidade de São Paulo.

Programa de Saúde Coletiva.

Orientador: Nelson da Cruz Gouveia.

Descritores: 1.Poluição do ar 2.Classe social 3. Mortalidade 4. Recém-nascido prematuro 5. Recémnascido de baixo peso 6.Epidemiologia

$\mathrm{USP} / \mathrm{EM} / \mathrm{DBD}-115 / 20$

Responsável: Erinalva da Conceiçāo Batista, CRB-8 6755 


\section{DEDICATÓRIA}

À minha esposa 


\section{AGRADECIMENTOS}

Ao meu orientador, Nelson Gouveia, pela oportunidade e confiança.

À professora Marcia Furquim por ter acreditado em mim desde o começo.

À colega Maria Antonietta Mascolli pela ajuda na revisão.

Ao colega Lucas Soriano Martins pela ajuda na confecção dos mapas.

Aos professores Paulo Rossi Menezes, Washington Leite Junger e Gleice Margarete de Souza Conceição pelas valorosas contribuições no exame de qualificação de Doutorado.

À minha esposa pela ajuda nas citações e referências.

À Coordenação de Aperfeiçoamento de Pessoal de Nível Superior (CAPES) pela bolsa de doutorado. 
Esta tese está de acordo com as seguintes normas, em vigor no momento desta publicação.

Referências: adaptado de International Committee of Medical Journals Editors (Vancouver). Universidade de São Paulo. Faculdade de Medicina. Divisão de Biblioteca e Documentação. Guia de apresentação de dissertações, teses e monografias. Elaborado por Annelise Carneiro da Cunha, Maria Julia de A. L. Freddi, Maria F. Crestana, Marinalva de Souza Aragão, Suely Campos Cardoso, Valéria Vilhena, 3ํㅡㄹ ed. São Paulo: Divisão de Bibliotecas e Documentação; 2011.

Abreviaturas dos títulos dos periódicos de acordo com List of Journals Indexed in Index Medicus. 


\section{SUMÁRIO}

LISTA DE SIGLAS, ABREVIATURAS E SÍMBOLOS

LISTA DE GRÁFICOS

LISTA DE FIGURAS

LISTA DE TABELAS

RESUMO

ABSTRACT

1 INTRODUÇÃO 1

2 REVISÃO DA LITERATURA

2.1 Efeitos da poluição do ar na mortalidade.............................

2.2 Efeitos da poluição do ar nos desfechos negativos da

gravidez.

2.3 Modificação de efeito da condição socioeconômica na associação entre poluição e eventos adversos na saúde 11

3 OBJETIVOS

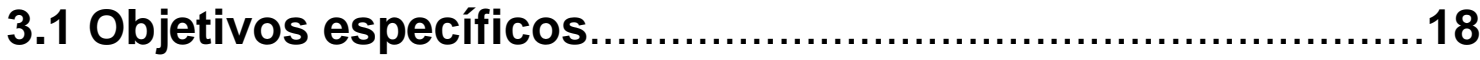

4 METODOLOGIA

4.1 Medição da poluição atmosférica.......................................19

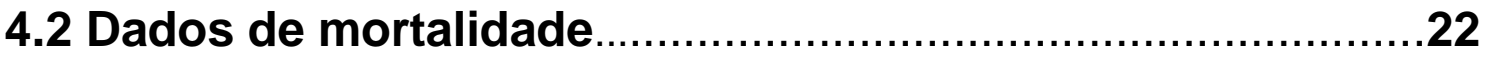

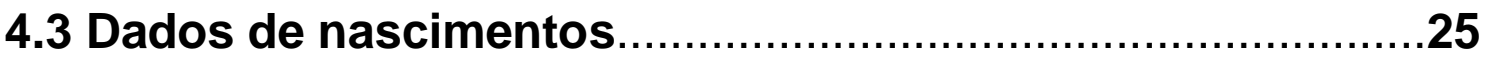

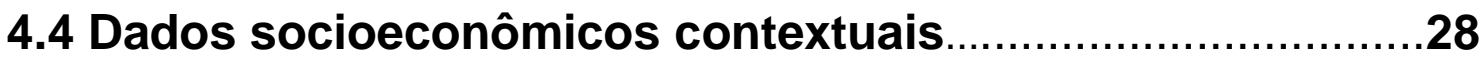

4.5 Dados meteorológicos.........................................................

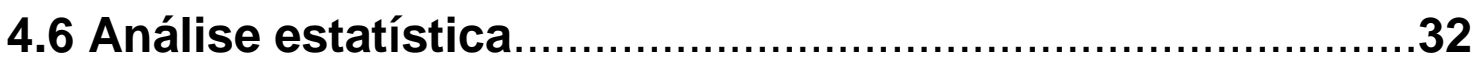

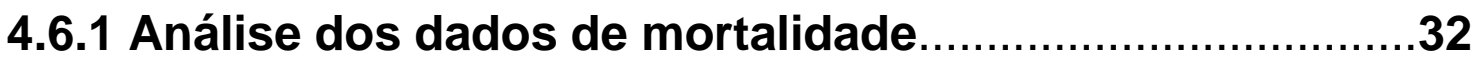

4.6.2 Análise dos dados de eventos adversos da gestação.....33

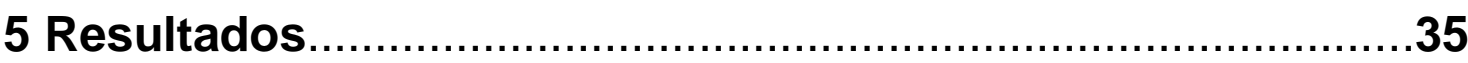

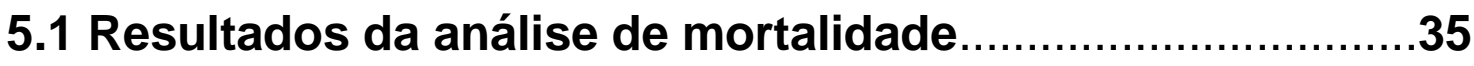


5.2 Resultados da análise dos desfechos adversos da gestação...................................................................

6 DISCUSSÃO............................................................... 47

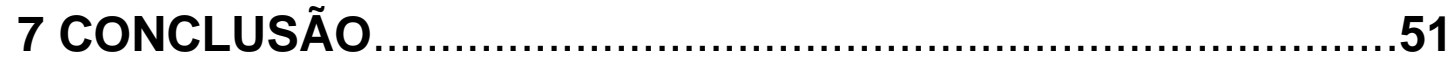

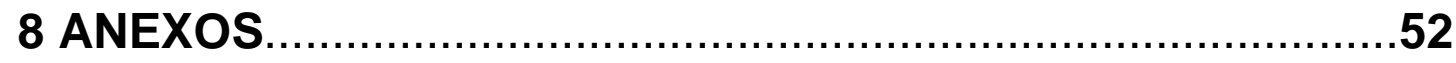

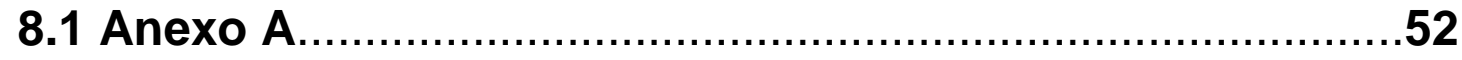

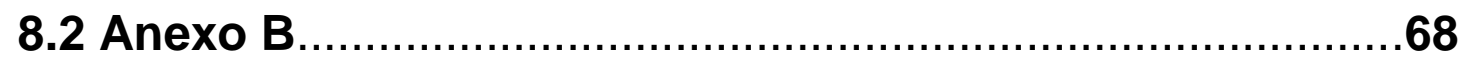

9 REFERÊNCIAS .......................................................92

10 BIBLIOGRAFIA CONSULTADA ....................................104

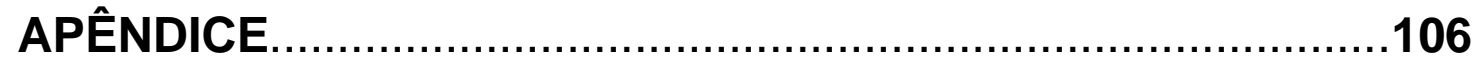




\title{
LISTA DE SIGLAS, ABREVIATURAS e SÍMBOLOS
}

\author{
AVC Acidente vascular cerebral \\ BPN Baixo peso ao nascer \\ BPNT Baixo peso em nascimento a termo \\ CBO Classificação Brasileira de Ocupações \\ CEP Código de Endereçamento Postal \\ CETESB Companhia Ambiental do Estado de São Paulo \\ CFM Conselho Federal de Medicina \\ CID 10 Código Internacional de Doenças décima versão \\ CNES Cadastro Nacional de Estabelecimentos de Saúde \\ CO Monóxido de carbono \\ CONAMA Conselho Nacional do Meio Ambiente \\ COREN Conselho Regional de Enfermagem \\ CPF Cadastro de Pessoa Física \\ CREMERS Conselho Regional de Medicina do Estado do Rio Grande do Sul \\ DN Declaração de Nascido Vivo \\ DP Desvio-padrão \\ DPOC Doença pulmonar obstrutiva crônica \\ DUM Data da última menstruação \\ EUA Estados Unidos da América \\ FUNASA Fundação Nacional de Saúde
}




\begin{tabular}{|c|c|}
\hline GEVIMS & Grupo de Estatísticas Vitais do Ministério da Saúde \\
\hline HAP & Hidrocarbonetos aromáticos policíclicos \\
\hline $\mathrm{HbCO}$ & Carboxihemoglobina \\
\hline $\mathrm{HR}$ & Hazard Ratio \\
\hline IBGE & Instituto Brasileiro de Geografia e Estatística \\
\hline IC95\% & Intervalo de confiança de $95 \%$ \\
\hline IMC & Índice de massa corporal \\
\hline IML & Instituto Médico Legal \\
\hline$\mu \mathrm{m}$ & Micrometro \\
\hline $\mathrm{MP}_{2.5}$ & Material particulado com diâmetro inferior a 2.5 micrometros \\
\hline $\mathrm{MP}_{10}$ & Material particulado com diâmetro inferior a 10 micrometros \\
\hline $\operatorname{Max}$ & Máximo amostral \\
\hline Min & Mínimo amostral \\
\hline NO & Óxido nítrico \\
\hline $\mathrm{NO}_{2}$ & Dióxido de nitrogênio \\
\hline $\mathrm{NO}_{\mathrm{x}}$ & Óxidos de nitrogênio \\
\hline NPT & Nascimento pré-termo \\
\hline $\mathrm{O}_{2}$ & Oxigênio \\
\hline $\mathrm{O}_{3}$ & Ozônio \\
\hline OMS & Organização Mundial de Saúde \\
\hline OR & Odds Ratio \\
\hline PIG & Pequeno para idade gestacional \\
\hline
\end{tabular}


PPB Partes por bilhão

PRO-AIM Programa de Aperfeiçoamento das Informações de Mortalidade

RG Registro Geral

RMSP Região metropolitana de São Paulo

RR Risco relativo

$\%$ Porcentagem de aumento do risco relativo

SEADE Fundação Sistema Estadual de Análise de Dados

SES Status socioeconômico

SESV Status socioeconômico de vizinhança

SIM Sistema de Informação de Mortalidade

SINASC Sistema de Informação Sobre os Nascidos Vivos

SMDU Secretaria Municipal de Desenvolvimento Urbano

$\mathrm{SO}_{2} \quad$ Dióxido de enxofre

SUS Sistema Único de Saúde

SVO Serviço de Verificação de Óbito

UF $\quad$ Unidade Federativa

USP Universidade de São Paulo

WHO World Health Organization 


\section{LISTA DE GRÁFICOS}

Grafico 1: Modificação de efeito da educação materna no risco de prematuridade associado a um aumento de $10 \mu \mathrm{g} / \mathrm{m}^{3}$ de cada poluente para todos os trimestres e todo o período gestacional, São Paulo, 2011-2016 44

Grafico 2: Modificação de efeito do SESV no risco de prematuridade associado a um aumento de $10 \mu \mathrm{g} / \mathrm{m}^{3}$ de cada poluente para todos os trimestres e todo o período gestacional, São Paulo, 20112016

Gráfico 3: Modificação de efeito da educação materna no risco de BPNT associado a um aumento de $10 \mu \mathrm{g} / \mathrm{m}^{3}$ de cada poluente para todos os trimestres e todo o período gestacional, São Paulo, 20112016

Gráfico 4: Modificação de efeito do SESV no risco de BPNT associado a um aumento de $10 \mu \mathrm{g} / \mathrm{m}^{3}$ de cada poluente para todos os trimestres e todo o período gestacional, São Paulo, 20112016 


\section{LISTA DE FIGURAS}

Figura 1 - Distribuição das Estações de Monitoramento de Ar do Estado e da Região Metropolitana de São Paulo, CETESB,

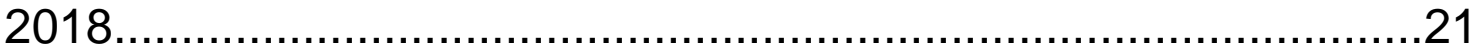

Figura 2 - Distribuição espacial dos distritos de São Paulo, segundo status socioeconômico de vizinhança..........................................31 


\section{LISTA DE TABELAS}

Tabela 1: Estatísticas descritivas da mortalidade da população de estudo, por causa de morte, São Paulo, 2011 - 2015.....................36

Tabela 2: Medidas descritivas dos poluentes e medições meteorológicas, São Paulo, 2011 - 2015....................................37

Tabela 3: Correlação de Pearson entre os poluentes e parâmetros

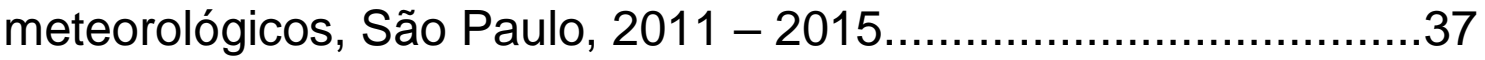

Tabela 4: Mudança percentual no risco de mortalidade associada a um aumento de $10 \mu \mathrm{g} / \mathrm{m}^{3}$ na concentração dos poluentes com IC95\% para cada desfecho São Paulo, 2011-2015. 38

Tabela 5: Alteração percentual do efeito da poluição do ar na mortalidade por causas não acidentais devido à educação e SESV associada a um aumento de $10 \mu \mathrm{g} / \mathrm{m}^{3}$ nos poluentes, com IC95\%, São Paulo, 2011-2015 39

Tabela 6: Alteração percentual do efeito da poluição do ar na mortalidade por causas cardiovasculares devido à educação e SESV associada a um aumento de $10 \mu \mathrm{g} / \mathrm{m}^{3}$ nos poluentes, com IC95\%, São Paulo, 2011-2015

Tabela 7: Alteração percentual do efeito da poluição do ar na mortalidade por causas respiratórias devido à educação e SESV associada a um aumento de $10 \mu \mathrm{g} / \mathrm{m}^{3}$ nos poluentes, com IC95\%, São Paulo, 2011-2015 
Tabela 8: Estatísticas descritivas e OR com IC95\% provenientes da regressão logística multinível univariada da associação entre desfechos adversos da gravidez e covariáveis para a população de estudo, São Paulo, 2011-2016.

Tabela 9: Odds Ratios (IC95\%) dos modelos de regressão logística multinível, ajustados por covariáveis, São Paulo, 20112016. 


\section{Resumo}

Nascimento FP. A condição socioeconômica altera os efeitos da poluição do ar na saúde? [tese]. São Paulo: Faculdade de Medicina, Universidade de São Paulo; 2020.

A poluição do ar é responsável por vários efeitos nocivos à saúde dos seres humanos. Estima-se que $90 \%$ da população mundial esteja exposta a algum tipo de agente tóxico no ar e a desigualdade social pode agravar os efeitos deletérios causados por essa poluição. O objetivo deste estudo foi examinar se o efeito da poluição do ar na saúde da população é alterado devido à diferença no nível socioeconômico. Primeiramente apresenta-se uma revisão da literatura abordando os efeitos da exposição à poluição do ar na mortalidade por causas cardiorrespiratórias e por todas as causas não acidentais e em eventos adversos da gestação e em seguida descrevem-se os estudos sobre a modificação de efeito da condição socioeconômica nestas relações. Em seguida, avaliou-se a modificação de efeito da condição socioeconômica na relação entre poluição do ar e mortalidade por causas não acidentais e cardiorrespiratórias. Os resultados indicaram a existência de modificação de efeito para todos os poluentes medidos, sendo a população com piores indicadores socioeconômicos a mais afetada. Finalmente, foi avaliada a modificação de efeito da condição socioeconômica na relação entre poluição e desfechos negativos da gravidez. Os resultados novamente indicaram a presença de modificação de efeito dos indicadores socioeconômicos individuais e contextuais na associação entre poluentes do ar e desfechos adversos da gravidez. Em conclusão, foram encontradas evidências de que as populações com pior acesso à educação e vivendo em regiões menos assistidas têm maior risco de mortalidade por causas não acidentais e cardiorrespiratórias e de desfechos negativos na gravidez devidos à poluição atmosférica.

Descritores: Poluição do ar; Classe social; Mortalidade; Recém-nascido Prematuro; Recém-nascido de baixo peso; Epidemiologia. 


\section{Abstract}

Nascimento FP. Does socioeconomic status modify the effect of air pollution on health outcomes? [thesis]. São Paulo: "Faculdade de Medicina, Universidade de São Paulo"; 2020.

Air pollution is responsible for several harmful effects on human health. It is estimated that $90 \%$ of the world population is exposed to some type of toxic agent and social inequality can aggravate the harmful effects caused by air pollution. The objective of this study was to examine whether the effect of air pollution on the population's health is altered due to differences in socioeconomic status. First, a literature review is presented addressing the effects of exposure to air pollution on mortality from cardiorespiratory causes and from all non-accidental causes and on adverse pregnancy events, and then studies on the effect modification by socioeconomic condition in these relationships. Then, the modification of the effect by socioeconomic status on the relationship between air pollution and mortality from non-accidental and cardiorespiratory causes was evaluated. The results indicated the existence of an effect modification for all measured pollutants, with the population with the worst socioeconomic indicators being the most affected. Finally, the effect modification of socioeconomic status on the relationship between pollution and adverse pregnancy outcomes was accessed. The results again indicated the presence of effect modification for all pollutants by individual and contextual socioeconomic indicators. In conclusion, evidence was found that populations with poorer access to education and living in less assisted areas have a higher risk of mortality from non-accidental and cardiorespiratory causes and from negative outcomes in pregnancy due to air pollution.

Descriptions: Air pollution; Social class; Mortality; Infant, premature; Infant, low birth weight; Epidemiology. 


\section{INTRODUÇÃO}

A poluição atmosférica tem sido identificada como um grave problema para a saúde pública desde o começo do século XX. As duas principais fontes antropogênicas de emissão associadas à contaminação do ar são os processos industriais, presentes desde a revolução industrial, e os veículos automotores movidos a combustíveis derivados de petróleo, especialmente a partir da metade do século XX (Braga et al., 2007). Estima-se que atualmente $90 \%$ da população mundial respire ar poluído'.

Dentre os poluentes mais frequentemente associados a reações adversas à saúde humana temos o material particulado de diâmetros inferiores a 2,5 e 10 $\mu \mathrm{m}\left(\mathrm{MP}_{2.5}\right.$ e $\mathrm{MP}_{10}$ respectivamente), o ozônio $\left(\mathrm{O}_{3}\right)$, o dióxido de nitrogênio $\left(\mathrm{NO}_{2}\right)$, o monóxido de carbono $(\mathrm{CO})$ e o dióxido de enxofre $\left(\mathrm{SO}_{2}\right)$.

As principais fontes do material particulado são os automóveis, as indústrias e a construção civil. Os efeitos do $\mathrm{MP}_{10}$ na saúde incluem a diminuição da função e do crescimento pulmonar, acidente vascular cerebral (AVC), as doenças cardíacas, os cânceres de pulmão, a mortalidade infantil por causas respiratórias e o aumento dos sintomas ligados à asma (WHO, 2008). O MP 10 é estimado como sendo responsável por cerca de $1 \%$ das mortes por infecção respiratória aguda nas áreas urbanas (Cohen et al., 2005) e recentemente foi classificado como carcinogênico de grupo 1 para populações humanas (Moore et al., 2017). Foi sugerido que o MP10 é o principal responsável pela irritação crônica do trato respiratório e diminuição da taxa de limpeza dos pulmões, o que explicaria parcialmente o aumento de mortes por causas respiratórias e a crescente incidência de câncer de pulmão. Os cânceres têm um período de latência muito grande e diversas possíveis causas que dificultam a medição do efeito isolado da poluição na sua incidência (Eitan et al., 2010). A Organização

\footnotetext{
${ }^{1}$ Organização Mundial da Saúde (OMS). "Nove em cada dez pessoas em todo mundo respiram ar poluído". https://www.paho.org/bra/index.php?option=com_content\&view=article\&id=5654:nove-emcada-dez-pessoas-em-todo-o-mundo-respiram-ar-poluido\&ltemid=839. Acesso em 1 Jun 2018.
} 
Mundial da Saúde (OMS) recomenda que os níveis médios anuais de $\mathrm{MP}_{10}$ devam ser inferiores a $20 \mu \mathrm{g} / \mathrm{m}^{3}$ e os de $\mathrm{MP}_{2.5}$ inferiores a $10 \mu \mathrm{g} / \mathrm{m}^{32}$.

O ozônio é produzido quando os óxidos de nitrogênio $\left(\mathrm{NO}_{\mathrm{x}}\right)$ e os compostos orgânicos voláteis reagem quimicamente sob a luz do sol. Seus efeitos conhecidos são a diminuição do crescimento pulmonar, aumento nas crises de asma, o aumento nas hospitalizações por todas as causas respiratórias, danos ao trato respiratório, decaimento do funcionamento do pulmão, inflamações e comprometimento do aparelho respiratório, acarretando doenças como asma (Li S et al., 2016).

O dióxido de nitrogênio é resultado da queima dos combustíveis derivados de petróleo e da reação do óxido nítrico (NO) com o oxigênio $\left(\mathrm{O}_{2}\right)$, sendo utilizado amplamente como indicador de tráfico veicular (Ghosh et al., 2013; Pinault et al., 2016). Seus efeitos à saúde conhecidos são aumento nos sintomas da asma e diminuição da função pulmonar (WHO, 2008).

O monóxido de carbono (CO) é formado quando os combustíveis a base de carbono não são completamente queimados e são emitidos por veículos automotores mais do que por qualquer outra fonte. Seus efeitos à saúde são o aumento do número das hospitalizações por asma, dores de cabeça e aumento da frequência de doenças do trato respiratório baixo, além da formação da carboxihemoglobina $(\mathrm{HbCO})$ no sangue, que evita a ligação do oxigênio na corrente sanguínea, podendo ser fatal em casos de intoxicação (WHO, 2008).

Já o dióxido de enxofre é emitido principalmente por indústrias de energia e de aço. Seus efeitos à saúde humana são o aumento do número de hospitalizações por asma e o aumento da frequência das doenças do trato respiratório baixo (WHO, 2008).

Os efeitos da poluição do ar na saúde das pessoas ocorrem mesmo quando seus níveis estão abaixo dos permitidos por lei (Bakonyi et al., 2004). Diversos estudos mostraram uma associação positiva entre morbidade e

\footnotetext{
2 OMS. "Nove em cada dez pessoas em todo mundo respiram ar poluído". https://www.paho.org/bra/index.php?option=com_content\&view=article\&id=5654:nove-em-cada-dezpessoas-em-todo-o-mundo-respiram-ar-poluido\&Itemid=839. Acesso em 1 Jun 2018.
} 
mortalidade e poluição do ar em relação a problemas respiratórios (Braga et al., 1999; Lin et al., 1999; Pinault et al., 2016).

Inúmeros estudos epidemiológicos vêm avaliando os efeitos da poluição do ar na saúde dos seres humanos. Em um estudo realizado em Israel foi reportado um risco relativo $12 \%$ maior de incidência de câncer de pulmão em homens para um aumento $1 \mu \mathrm{g} / \mathrm{m}^{3}$ de $\mathrm{MP}_{10}$ (Eitan et al., 2010). Um estudo realizado por Brook e colaboradores (2008) sugere que o $\mathrm{MP}_{10}$ também é um dos responsáveis pelo aumento de casos de doenças cardiovasculares e de diabetes tipo 2. Os resultados do estudo de Krämer et al. (2010) na Alemanha indicam que existe associação entre exposição a poluição do ar por derivados de petróleo e diabetes tipo 2 em mulheres. Foi reportado um Hazard Ratio (HR) para diabetes de 1,15 devido à exposição veicular tanto de $\mathrm{MP}_{10}$ quanto de $\mathrm{NO}_{2}$. Anemia também já foi associada positivamente com exposição a $\mathrm{MP}_{2.5}$ e $\mathrm{NO}_{2}$ em idosos em um estudo nos Estados Unidos da América (EUA). Resultados dessa coorte mostraram um aumento de $33 \%$ na prevalência da doença para um aumento de $3,9 \mu \mathrm{g} / \mathrm{m}^{3}$ na média móvel anual de $\mathrm{MP}_{2.5}$ e de $43 \%$ para um aumento de 9,6 partes por bilhão (ppb) de $\mathrm{NO}_{2}$ (Honda et al.,2017). Em seu estudo na Coréia do Sul, Kim e colaboradores (2015) avaliaram os efeitos da exposição horária da população ao $\mathrm{MP}_{10}$ e $\mathrm{O}_{3}$ e encontraram uma associação positiva entre crises de asma e maior exposição a esses poluentes. No Brasil, o estudo de Ferreira et al. (2016) identificou associação positiva entre emissão de níveis elevados de $\mathrm{MP}_{10}$ e $\mathrm{MP}_{2.5-10}$ e admissões hospitalares por problemas respiratórios e $\mathrm{MP}_{2.5}$ com admissões por problemas circulatórios.

A poluição do ar ambiente também tem sido consistentemente associada a eventos adversos no nascimento como parto prematuro (definido como parto antes das 37 semanas de gestação) e baixo peso ao nascer (BPN, definido como peso ao nascer inferior a $2500 \mathrm{~g}$ ), que são considerados importantes indicadores epidemiológicos da saúde dos recém-nascidos (Stieb et al., 2016). Recémnascidos prematuros apresentam maiores taxas de mortalidade infantil, que aumentam à medida que a idade gestacional diminui (Mathews; MacDorman, 2012), enquanto o BPN vem sendo associado a um maior risco de síndrome metabólica, diabetes tipo 2, doenças cardiovasculares e asma na infância 
(Chernausek, 2012; Caudri et al., 2007). Foi relatado na literatura que idade materna, escolaridade, renda, pré-natal e etnia são fatores de risco para prematuridade (Yi et al., 2010) e baixo peso em nascimento a termo (BPNT, peso ao nascer inferior a $2500 \mathrm{~g}$ nas gestações de 37 a 42 semanas) (Gouveia et al., 2004; Romão et al., 2013).

A exposição crônica a compostos tóxicos da poluição do ar durante a gravidez pode induzir inflamação, afetar a hemodinâmica da placenta e o transporte de oxigênio e nutrientes para o feto, levando à restrição de crescimento intrauterino e BPNT (Laurent et al., 2014; Li L et al., 2016). O MP 10 é composto por muitos produtos químicos essenciais que absorvem hidrocarbonetos aromáticos policíclicos (HAP) e outros compostos orgânicos que podem gerar várias formas de inflamação (Delfino et al., 2010) e sua exposição a eles foi associada a um maior risco de prematuridade (Schifano et al., 2010) e BPNT (Gray et al., 2014; Stieb et al., 2016). Revisões sistemáticas e meta-análises indicam que essa associação existe e é biologicamente plausível (Dadvand et al., 2013; Šrám et al., 2005; Stieb et al., 2012).

A condição socioeconômica também tem efeito na saúde humana. Vários estudos documentaram que indivíduos com piores indicadores sociais têm menos acesso a planos de saúde e, geralmente, estilos de vida menos saudáveis (Lynch et al., 2000; Fecht et al., 2015). Estudos também demonstraram a existência de um gradiente socioeconômico no qual, a cada passo hierárquico acima, as pessoas têm melhores indicadores de saúde do que as que estão um degrau abaixo. O status socioeconômico (SES) pode ser medido tanto individualmente quanto contextualmente ou por vizinhança (bairros, setores censitários, distritos). Indicadores individuais de SES incluem nível educacional, renda, ocupação entre outros, enquanto indicadores de SES de vizinhança incluem, percentual de residências com acesso a esgoto, percentual de residências com acesso a água potável, renda média de determinado setor censitário, taxa média de alfabetização entre outros, e cada um desses indicadores têm efeito direto e indireto na saúde (O’Neill et al., 2003). 
Em estudos mais recentes têm havido um crescente interesse em avaliar como a poluição do ar e os fatores socioeconômicos interagem para explicar as disparidades na saúde da população. Hipóteses são levantadas sobre a interação entre os efeitos da poluição na saúde e a condição socioeconômica. A primeira sugere que pessoas mais pobres tendem a trabalhar em locais onde há maior exposição a agentes tóxicos. A segunda sugere que a menor escolaridade associada com estilos de vida menos saudáveis e o menor acesso a serviços de saúde fariam com que indivíduos com menor SES se tornassem mais vulneráveis aos efeitos deletérios da poluição do ar (Wong et al., 2008; Deguen et al., 2010). Menor escolaridade e renda já foram associadas com pior nível de saúde, incluindo maior taxa de mortalidade e maior exposição à poluição, que é a definição de desigualdade ambiental (Lynch et al., 2000; Fecht et al., 2015).

O'Lenick et al. (2017) encontraram maior efeito da poluição do ar nos casos de asma em crianças que moravam em áreas com piores indicadores socioeconômicos nos EUA, assim como o estudo de Yap et al. (2013) que mostrou que nos distritos com piores SES ocorreram mais admissões em hospitais por causas respiratórias em crianças devido ao efeito do $\mathrm{MP}_{2.5}$.

Este estudo tem como objetivo contribuir com o embate cientifico acerca da modificação de efeito da condição socioeconômica na associação entre exposição do ar-desfechos negativos na saúde no município de São Paulo. Para tal, no capítulo 2 foi realizada uma revisão da literatura abordando os principais estudos que reportaram associação entre exposição à poluição do ar e mortalidade e também eventos adversos na gestação devido à exposição a poluentes atmosféricos e, por fim, os principais estudos que investigaram a modificação de efeito da condição socioeconômica na mortalidade e nos desfechos negativos da gravidez. No capítulo 3 apresenta-se os objetivos gerais e específicos da tese. Já o capítulo 4 trata da metodologia empregada, destacando as fontes e análises dos dados que foram utilizados neste estudo. São abordadas as fontes de dados de poluição do ar, mortalidade, nascimentos, dados socioeconômicos e meteorológicos. Em seguida, são apresentados os métodos estatísticos que foram aplicados para a análise de cada desfecho de interesse. No capítulo 5 são apresentados os resultados das análises para os 
dados de mortalidade e eventos adversos da gestação, separadamente. No capítulo 6 discute-se os resultados apresentados no capítulo 5, comparando-os com a literatura nacional e internacional, apresenta-se mecanismos e causas plausíveis para os achados, limitações do estudo e recomendações de investigação para estudos futuros. No capítulo 7 são apresentadas as conclusões dos achados desta tese. O capítulo 8 apresenta os dois artigos produzidos sendo um submetido, mas ainda não aceito para publicação. 


\section{REVISÃO DA LITERATURA}

\subsection{Efeitos da poluição do ar na mortalidade}

As evidências cientificas de inúmeros estudos epidemiológicos apontam que a poluição do ar tem impacto direto tanto na mortalidade da população quanto na diminuição de anos de vida. Vários estudos de coorte encontraram associações entre exposição a determinados poluentes do ar e mortalidade, como o de Carey e colaboradores (2013), que encontraram associações positivas entre exposição a $\mathrm{MP}_{2.5}, \mathrm{NO}_{2}$ e $\mathrm{SO}_{2}$ e mortalidade por todas as causas (Hazard Ratio (HR) = 1,02, 1,03 e 1,04 respectivamente), mortalidade por causas respiratórias ( $H R=1,09$ para todos os poluentes) e por câncer de pulmão ( $H R=$ 1,02, 1,06 e 1,05 respectivamente), em uma coorte na Inglaterra. Também na Inglaterra, Maheswaran e colaboradores (2010) estudaram a sobrevida de pacientes após acidente vascular cerebral (AVC) em uma coorte. A principal causa da diminuição da sobrevida nos pacientes foi a maior exposição ao $\mathrm{MP}_{10}$ e ao $\mathrm{NO}_{2}$. Um aumento médio de $10 \mu \mathrm{g} / \mathrm{m}^{3}$ na exposição de $\mathrm{NO}_{2}$ foi associado com maior chance de mortalidade ( $H R=1,41$ [IC95\% $=1,14-1,75]$ ), enquanto que o efeito para o $\mathrm{MP}_{10}$ um aumento na exposição de $10 \mu \mathrm{g} / \mathrm{m}^{3}$ foi $\mathrm{HR}=1,81$ $[$ IC95\% $=1,05-3,13]$. Os autores desse estudo levantaram a hipótese de que 0 AVC pode comprometer a função respiratória, deixando os indivíduos acometidos ainda mais susceptíveis aos efeitos da poluição do ar. Na Itália, Cesaroni e colaboradores (2013) reportaram, em um estudo de coorte, que exposição crônica a $\mathrm{MP}_{2.5}$ e NO 2 foi associada a um aumento para mortalidade por todas as causas não acidentais ( $H R=1,03$ e 1,04 respectivamente). A associação foi ainda maior para doenças isquêmicas do coração, doenças cardiovasculares e câncer de pulmão. Os autores desse estudo sugerem que a poluição do ar age não como causador, mas como catalizador de comorbidades pré-existentes. Na China, o estudo de coorte retrospectiva realizado por Zhang et al. (2011) reportou que, um aumento médio de $10 \mu \mathrm{g} / \mathrm{m}^{3}$ na concentração de $\mathrm{MP}_{10}$ leva a um aumento no risco de mortes por eventos cardiovasculares (HR= $1,55[1,51-1,60])$, assim como um aumento de $10 \mu \mathrm{g} / \mathrm{m}^{3}$ nas concentrações médias de $\mathrm{NO}_{2}(\mathrm{HR}=2,46[\mathrm{IC} 95 \%=2,31-2,63])$. Os autores relatam que os mecanismos que fazem com que as partículas da poluição do ar causem eventos 
cardíacos não são claros, mas levantam a hipótese de que a poluição aumente a taxa de coagulação do sangue, levando a liberação de células inflamatórias, o que poderia resultar na coagulação do sangue e desencadear um evento cardiovascular no indivíduo.

Na Suécia, Rosenlund e colaboradores (2009) reportaram, em um estudo caso-controle populacional, uma associação positiva entre exposição a 5 anos de $\mathrm{NO}_{2}$ gerado por tráfego veicular e mortalidade por infarto do miocárdio $(\mathrm{OR}=$ 1,23 [IC95\% = 1,15 - 1,32]). Os autores sugerem que existe uma relação entre a exposição ao poluente e arritmia seguida por infarto, mas relatam a dificuldade de afirmar uma relação de causalidade nessa associação. Na China, Qiu e colaboradores (2015), em um estudo "case-only", encontraram aumento na mortalidade por causas cardiorrespiratórias entre 1,6\% e 2,3\% para um aumento médio de $10 \mu \mathrm{g} / \mathrm{m}^{3}$ de $\mathrm{MP}_{10}$ ou $\mathrm{NO}_{2}$. Os autores sugerem que esses poluentes agravam comorbidades prévias dos indivíduos como AVC, infarto do miocárdio, pneumonias e doença pulmonar obstrutiva crônica (DPOC).

Li C. e colaboradores (2016), em um estudo ecológico, mediram e compararam a expectativa de vida de municípios com baixa e alta emissão de $\mathrm{O}_{3}$ nos EUA e descobriram que para um aumento de 5 ppb na exposição a esse poluente há uma perda média de 0,25 anos de vida para homens e 0,21 anos para mulheres, mesmo ajustado por outras variáveis. Em um estudo ecológico, na Espanha, Keijzer et al. (2017) encontraram uma redução média na expectativa de vida de 0,90, 0,13, 0,20 e 0,64 anos para um aumento de $5 \mu \mathrm{g} / \mathrm{m}^{3}$ na exposição de $\mathrm{MP}_{10}, \mathrm{NO}_{2}, \mathrm{O}_{3}$ ou $2 \mu \mathrm{g} / \mathrm{m}^{3}$ de $\mathrm{MP}_{2.5}$, respectivamente.

Esta revisão mostrou haver evidências muito fortes entre exposição a poluentes atmosféricos e mortalidade por todas as causas e causas cardiorrespiratórias, mas os mecanismos que regem essa associação ainda não foram completamente esclarecidos.

\subsection{Efeitos da poluição do ar nos desfechos adversos da gestação}

Dentre os possíveis eventos adversos da gestação, os mais comumente estudados são o BPN (que pode ser resultado de gestações prematuras e, a 
termo, pode ocorrer devido à restrição de crescimento intrauterino associada à condição de saúde da mãe, como pré-eclâmpsia e distúrbios da placenta) e os nascimentos prematuros (Laurent et al., 2014; Rich et al., 2009; Wu et al., 2009). A proporção de prematuridade está aumentando em vários países, incluindo o Brasil (Alencar et al., 2016), enquanto o BPN ocorre com mais frequência em países de baixa e média renda (OMS, 2014). No Brasil, o BPN apresenta uma tendência estacionária de cerca de 8\% dos nascidos vivos no período 2011-2015 (Henriques et al., 2019). Vários estudos epidemiológicos se propuseram a estudar a relação entre exposição à poluição do ar e desfechos negativos da gravidez, assim como os mecanismos que regem essa associação.

Em um estudo transversal realizado no Brasil, Romão et al. (2013) relataram associação entre maior exposição ao $\mathrm{MP}_{10}$ em todos os trimestres e risco de $\operatorname{BPN}(\mathrm{OR}=1,23,1,26,1,23$ para exposição no primeiro, segundo e terceiro trimestre respectivamente, para um aumento de $37,50 \mu \mathrm{g} / \mathrm{m}^{3}$ ). Os autores sugerem que o BPN ocorre devido ao subdesenvolvimento da placenta que seria consequência de problemas de coagulação e inflamação pulmonar resultantes da exposição a poluição do ar. Foi reportado, no Canadá, em outro estudo transversal, associação entre aumento médio de $10 \mu \mathrm{g} / \mathrm{m}^{3}$ na exposição a $\mathrm{MP}_{2.5}$ e recém-nascidos pequenos para a idade gestacional (PIG) $(\mathrm{OR}=1,04$ [IC95\% 1,01 - 1,07]) e BPN (-20,5g [IC95\% -24,7g;-16,4g]) (Stieb et al., 2016). Outro estudo transversal realizado nos EUA por Laurent et al. (2014) verificaram associação entre maior exposição ao $\mathrm{MP}_{2.5}$ durante toda a gestação e maior risco de BPN (OR = 1,020 [IC95\% 1,010 - 1,031]), mas não houve associação entre BPN e maior exposição a $\mathrm{O}_{3}$ e $\mathrm{NO}_{2}$. Ainda nos EUA, no estudo ecológico de Li $\mathrm{L}$ et al. (2016), maior exposição a $\mathrm{NO}_{2}$ e NOx foi associada a maior chance de BPN e esse efeito foi estatisticamente significativo nos três trimestres de gestação.

Rich et al. (2009) nos EUA, em seu estudo transversal, reportaram maior risco de recém-nascidos $\mathrm{PIG}$ devido à maior exposição ao $\mathrm{MP}_{2.5}$ durante o primeiro e terceiro trimestre de gestação. Na Austrália, o estudo case-crossover de Li S e colaboradores (2016) encontraram associação entre maior exposição a $\mathrm{NO}_{2}, \mathrm{SO}_{2}$ e $\mathrm{CO}$ com prematuridade, mas não com $\mathrm{MP}_{10}$ e $\mathrm{O}_{3}$. No Japão, Yorifuji et al. (2013) reportaram, em seu estudo transversal, risco aumentado em 1,5 
vezes para nascimentos prematuros e 1,2 vezes para BPN em gestantes morando a menos de 200 metros de vias com tráfego intenso de carros. Estes três últimos estudos sugerem que o potencial mecanismo de associação entre a poluição do ar e eventos adversos da gravidez se deve ao comprometimento da função da placenta (fornecimento de oxigênio e transporte de nutrientes) devido à ação inflamatória e coagulante que os poluentes causam no organismo.

Uma revisão de literatura realizada por Šrám et al. (2005) sugeriu que havia necessidade de novos estudos avaliando a exposição entre poluição do ar e desfechos negativos da gravidez. Os autores sugeriram que o mecanismo biologicamente plausível para explicar a associação entre exposição à poluição do ar e prematuridade e restrição do crescimento intrauterino seria por meio dos hidrocarbonetos aromáticos policíclicos (PAH) que teriam efeito antiestrogênico, que levaria a desfechos negativos da gravidez. Dadvand et al. (2013) reportaram resultados similares em sua meta-análise para BPNT, com OR de 1,03 (IC95\% 1,01 - 1,05) para um aumento de $10 \mathrm{\mu g} / \mathrm{m}^{3}$ na exposição ao $\mathrm{MP}_{10}$ e OR de 1,10 (IC95\% 1,03 - 1,18) para um aumento de $10 \mu \mathrm{g} / \mathrm{m}^{3}$ na exposição ao $\mathrm{MP}_{2.5}$ durante todo o período de gestação. Stieb et al. (2012) reportaram, em uma revisão sistemática com meta-análise de 62 estudos, OR de 1,10 (IC95\% 1,05 1,15) para chance de BPN para um aumento de $20 \mu \mathrm{g} / \mathrm{m}^{3}$ de MP 10 e OR de 1,06 (IC95\% 1,03 - 1,11) de prematuridade para um aumento de $20 \mu \mathrm{g} / \mathrm{m}^{3}$ de $\mathrm{MP}_{10}$ durante a gestação inteira. Para os demais poluentes $\left(\mathrm{CO}, \mathrm{NO}_{2}, \mathrm{O}_{3}, \mathrm{MP}_{2.5}, \mathrm{SO}_{2}\right)$ os resultados foram bastante heterogêneos. Os autores dessa revisão também destacam o fato de que os efeitos encontrados em trimestres separadamente têm magnitude inferiores aos que consideram todo o período gestacional.

A literatura científica também tem identificado associação entre exposição a poluição do ar durante trimestres específicos e durante todo o período gestacional e desfechos negativos da gravidez, mas os mecanismos que regem essa relação e quais os períodos críticos de exposição ainda não são compreendidos completamente. 


\subsection{Modificação de efeito da condição socioeconômica na associação entre poluição e eventos adversos na saúde}

Para analisar a modificação de efeito da condição socioeconômica na associação poluição do ar-mortalidade, utilizou-se uma estratégia de busca nas bases de dados eletrônicas MEDLINE, EMBASE e Web of Science. Houve grande heterogeneidade entre os artigos sobre a composição dos indicadores socioeconômicos. Foram mencionados educação, renda, índices de privação sobre determinada área censitária, status ocupacional e combinações entre mais de um indicador. A métrica utilizada para comparar o risco de cada poluente também foi diversa. Alguns artigos reportaram o risco para um aumento de 10 $\mu \mathrm{g} / \mathrm{m}^{3}$ do poluente, outros para um aumento do intervalo interquartil e algumas variações de exposição por partes por bilhão (ppb).

Os resultados de alguns estudos corroboram com a hipótese de que indivíduos com condição socioeconômica mais desfavorável estão expostos a níveis mais elevados de poluição do ar, como o estudo realizado na Coréia do Sul que mostrou que pessoas com melhor status socioeconômico e vivendo com menor número de indivíduos estavam expostos a menores níveis de poluição (Choi et al., 2016). No Canadá, Pinault et al. (2017b) relataram que a população de imigrantes e de minorias estão mais expostas à poluição em relação à população branca (média de 1,61 $\mu \mathrm{g} / \mathrm{m}^{3}$ e $1,55 \mu \mathrm{g} / \mathrm{m}^{3}$ de $\mathrm{MP}_{2.5}$ respectivamente) devido à localização das indústrias em bairros mais pobres. Em um estudo conduzido por Fecht et al. (2015) foram encontrados níveis mais elevados de $\mathrm{MP}_{10}$ e $\mathrm{NO}_{2}$ em áreas com piores indicadores socioeconômicos na Inglaterra e Holanda. Na Inglaterra, os bairros mais desfavorecidos tinham níveis médios de $\mathrm{MP}_{10} 1,5 \mu \mathrm{g} / \mathrm{m}^{3}$ mais elevados e níveis de $\mathrm{NO}_{2} 4,4 \mu \mathrm{g} / \mathrm{m}^{3}$ maiores.

No Brasil, Bravo et al. (2016) reportaram menor mortalidade por causas cardiovasculares devido à exposição ao $\mathrm{MP}_{10}$ no grupo com maior escolaridade. Blanco-Becerra et al. (2014), em um estudo ecológico na Colômbia, reportaram que SES modifica o efeito da exposição ao MP 10 para todas as causas de morte e para as causas respiratórias, sendo os indivíduos que moram nos distritos mais pobres os mais vulneráveis (porcentagem de aumento no risco relativo $(\mathrm{RR} \%)=$ 
0,76\% [IC95\% $=0,27 \%-1,26 \%]$ nas áreas mais pobres contra -0,29\% [IC95\% $1,16-0,57]$ nas áreas mais ricas para todas as causas de morte). No México, Carbajal-Arroyo et al. (2010) reportaram que crianças que viviam em áreas desfavorecidas tinham maior risco de mortalidade por causas respiratórias quando expostas ao $\mathrm{MP}_{10}$ e $\mathrm{O}_{3}$. O OR para mortalidade por todas as causas por aumento de $38,7 \mathrm{\mu g} / \mathrm{m}^{3}$ de $\mathrm{MP}_{10}$ foi de 1,073 [IC95\% = 1,015 - 1,133] nas áreas mais desfavorecidas contra 0,931 [IC95\% $=0,855-1,014$ ] nas áreas menos desfavorecidas e para as causas respiratórias foi de 1,089 [IC95\% = 1,001 $1,186]$ nas áreas mais desfavorecidas contra 0,889 [IC95\% $=0,763-1,036]$ nas menos desfavorecidas. No Chile, Cakmak e colaboradores (2011) encontraram modificação de efeito dos poluentes $\mathrm{CO}, \mathrm{NO}_{2}, \mathrm{SO}_{2}, \mathrm{O}_{3}, \mathrm{MP}_{2.5}$ e $\mathrm{MP}_{10}$ na mortalidade por todas as causas de acordo com o nível educacional e a renda, sendo as classes mais pobres e com menor escolaridade aquelas sob maior risco. $\mathrm{O}$ aumento na mortalidade devido à exposição a esses poluentes variou entre 4 e $7 \%$ nos indivíduos com menor escolaridade contra 0,5 a 1,5\% entre os indivíduos com maior escolaridade. Na Espanha, Barceló et al. (2009) encontraram modificação do efeito da exposição ao $\mathrm{MP}_{10}$ na mortalidade por causas respiratórias nas localidades mais pobres apenas nos homens (52\% maiores nas regiões mais pobres). Na França, Deguen et al. (2015), em um estudo case-crossover, reportaram que a exposição ao $\mathrm{NO}_{2}$ aumentou a mortalidade por todas as causas nos indivíduos que moravam nos blocos censitários mais pobres. Um estudo ecológico realizado no País de Gales por Brunt et al. (2016) encontrou modificação de efeito devido ao SES de vizinhança para maior exposição ao $\mathrm{MP}_{10} \mathrm{em}$ todas as causas de morte e exposição ao $\mathrm{MP}_{2.5}$ nas causas respiratórias, sendo as áreas mais pobres as mais afetadas.

Nos EUA, Kloog et al. (2013), em um estudo de séries temporais, encontraram modificação de efeito devido ao nível educacional na associação entre maior exposição ao $\mathrm{MP}_{2.5}$ e desfechos cardiorrespiratórios $(\mathrm{OR}=1,4$ IC95\% 1,2 - 1,6 na categoria de educação mais alta contra OR = 1,9 IC95\% 1,6 - 2,1 na categoria mais baixa). Também nos EUA, um estudo de coorte em idosos realizado por Wang et al. (2017), reportou HR mais elevados para mortalidade por todas as causas nos indivíduos que viviam em localidades com 
piores indicadores socioeconômicos quando expostos a níveis mais altos de $M P_{2.5}$. Wong et al. (2008) encontraram, na China, diferenças significativas entre a mortalidade por causas não acidentais e cardiovasculares para indivíduos com piores indicadores socioeconômicos sob efeito da exposição a níveis elevados de $\mathrm{NO}_{2}, \mathrm{SO}_{2}, \mathrm{MP}_{10}$ e $\mathrm{O}_{3}$. Pinault et al. (2017), em um estudo de coorte realizado no Canadá, reportaram HR para mortalidade não acidental mais elevados devido à exposição ao $\mathrm{MP}_{2.5}$ nos indivíduos que viviam em localidades mais pobres (HR = 1,129 IC95\% 1,110 - 1,149). No estudo realizado em Hong Kong por Qiu et al. (2015), foi reportado que os indivíduos economicamente inativos tiveram um risco adicional do efeito dos poluentes $\mathrm{MP}_{10}, \mathrm{MP}_{2.5}, \mathrm{NO}_{2}$ e $\mathrm{SO}_{2}$ associados com a mortalidade por todas as causas. Em um estudo realizado na Nova Zelândia, Richardson et al. (2011) reportaram maior impacto da exposição ao $\mathrm{MP}_{10}$ nos indivíduos vivendo em áreas mais pobres na mortalidade por todas as causas. Também na Nova Zelândia, Hales et al. (2012) reportou menor OR para mortalidade por todas as causas relacionadas a maior exposição ao $\mathrm{MP}_{10}$ nos indivíduos com maior escolaridade.

Em outros estudos foram encontrados resultados opostos, nos quais indivíduos com melhor condição socioeconômica apresentaram maior chance de eventos adversos na saúde quando expostos à poluição do ar. Na China, Zhang et al. (2014) encontraram, em uma coorte retrospectiva, que indivíduos com nível educacional e de renda altos apresentaram maiores riscos relativos (RR) para doenças isquêmicas do coração ( $R R=1,74$ (IC95\% = 1,51 - 2,00) nos indivíduos com maior escolaridade contra 1,28 (IC95\% $=1,19-1,38)$ nos com menor escolaridade e $R R=1,88$ (IC95\% = 1,66 - 2,13) nos mais ricos contra $R R=1,21$ (IC95\% = 1,13 - 1,30) nos mais pobres) e doenças cardíacas (RR = 1,52 (IC95\% $=1,41-1,65)$ nos indivíduos com maior escolaridade contra $R R=1,19($ IC95\% $=1,15-1,22)$ nos indivíduos com menor escolaridade; $R R=1,65$ (IC95\% $=1,53$ - 1,78) nos indivíduos com maior renda contra 1,14 (IC95\% = 1,11 - 1,18) para os indivíduos com menor renda) para um aumento de $10 \mu \mathrm{g} / \mathrm{m}^{3}$ na exposição ao $M P_{10 .}$

Em outros estudos não foi encontrada a modificação de efeito devido à condição socioeconômica. O'Neill e colaboradores (2008) em um estudo 
multicêntrico envolvendo São Paulo, Cidade do México e Santiago, reportaram que um aumento de $10 \mu \mathrm{g} / \mathrm{m}^{3}$ no $\mathrm{MP}_{10}$ aumenta o risco na mortalidade por todas as causas não externas $(0,39 \%$ IC $95 \%=0,13 \%-0,65 \%$ no México, $1,04 \%$ IC95\% $=0,71 \%-1,38 \%$ em São Paulo e 0,61\% IC95\% = 0,40\% - 0,83\% em Santiago), mas nenhum gradiente foi encontrado para diferentes escolaridades. Chi et al. (2016) encontraram um risco 13\% maior para eventos cardiovasculares para um aumento médio de $5 \mu \mathrm{g} / \mathrm{m}^{3}$ de $\mathrm{MP}_{2.5}$ em mulheres nos EUA, mas o efeito não foi diferente nos indivíduos de diferentes SES. Eftim et al. (2008) reportaram, em seu estudo de coorte realizado nos EUA, associação entre maior exposição ao $\mathrm{MP}_{2.5}$ e aumento na mortalidade por todas as causas que não foi modificada por ajustes do SES de vizinhança em vários modelos de análise. Em outra coorte nos EUA, Jerret et al. (2009) reportaram aumento na mortalidade por causas respiratórias e cardiovasculares associadas com maior exposição ao $\mathrm{MP}_{2.5}$ e $\mathrm{O}_{3}$ e não houve mudança desse aumento entre indivíduos com baixa ou alta escolaridade. Ren et al. (2010) encontraram, nos EUA, associação entre aumento dos níveis médios de $\mathrm{O}_{3}$ e aumento do risco de todas as causas de morte, problemas respiratórios, diabetes, doenças cardiovasculares, doenças no coração, infarto do miocárdio e AVC, mas não encontraram modificação de efeito devido à condição socioeconômica. Na China, Dong e colaboradores (2012), em uma coorte retrospectiva em adultos, encontraram maior RR para mortalidade por causas respiratórias (1,67 e 2,97 para um aumento de $10 \mu \mathrm{g} / \mathrm{m}^{3}$ de $\mathrm{MP}_{10} \mathrm{e}$ $\mathrm{NO}_{2}$ ), mas não encontraram diferenças nos $\mathrm{RR}$ para diferentes faixas de renda e de educação. Em outra coorte retrospectiva realizada na China, Zhang et al. (2011) identificaram um aumento na mortalidade por causas cardiovasculares devido a maior exposição ao $\mathrm{MP}_{10}$ e $\mathrm{NO}_{2}$, cujo efeito não foi modificado devido às diferenças de renda e educação entre os indivíduos. Também na China, um estudo transversal de Ou e colaboradores (2008), reportou RR positivo entre mortalidade e exposição a $\mathrm{MP}_{10}$ e $\mathrm{NO}_{2}$, mas não encontrou diferenças quando comparou grupos de diferentes escolaridades e rendas. Outro estudo realizado na China por Chen et al. (2012), reportou aumento na mortalidade associado a maior exposição ao $\mathrm{MP}_{10}$ e o efeito foi pontualmente maior entre aqueles com menos anos de educação, mas a modificação de efeito não foi estatisticamente significativa. Na Itália, Faustini et al. (2016) reportaram, em seu estudo case- 
crossover, um aumento na mortalidade devido à exposição ao $\mathrm{MP}_{10}$ e ao $\mathrm{NO}_{2}$, mas não houve modificação desse efeito devido ao SES de vizinhança. Também na Itália, Cesaroni et al. (2013) reportaram que, em sua coorte, o aumento da exposição ao $\mathrm{NO}_{2}$ e ao $\mathrm{MP}_{2.5}$ aumentou o $\mathrm{HR}$ para as causas não acidentais de morte e não houve modificação de efeito devido ao nível de escolaridade. Ainda na Itália, Chiusolo et al. (2011) encontraram, em seu estudo case-crossover, associação entre maior exposição ao $\mathrm{NO}_{2}$ e aumento na mortalidade por causas não acidentais, respiratórias e cardiovasculares. O efeito, entretanto, não variou entre diferentes níveis de educação e renda por setor censitário. Em um estudo case-crossover realizado na Noruega, Madsen et al. (2012) reportaram aumento na mortalidade por todas as causas associado a maior exposição ao $\mathrm{MP}_{2.5}$ e ao $\mathrm{NO}_{2}$ que não foi modificado devido ao SES de vizinhança.

Nos estudos de eventos adversos na gravidez também houve grande heterogeneidade entre os artigos sobre a composição dos indicadores de SES. Foram mencionados educação materna, renda, índices de privação censitária, status ocupacional, status marital e combinações entre mais de um indicador. A métrica utilizada para comparar o risco de cada poluente também foi diversa. Alguns artigos reportaram o risco para um aumento de $10 \mu \mathrm{g} / \mathrm{m}^{3}$ do poluente, outros para um aumento do intervalo interquartil e algumas variações de exposição por ppb e também distância da residência da mãe à rodovia mais próxima.

Nos EUA, dois estudos (Li L et al., 2016; Laurent et al., 2014) relataram uma associação de exposição materna ao $\mathrm{NO}_{2}$ com BPNT que foi modificada pelo nível educacional, no qual mães com baixa escolaridade eram mais suscetíveis aos efeitos da poluição do ar. Também nos EUA, Padula e colaboradores (2014) constataram que a poluição do ar gerada pelo tráfego aumentou a chance de prematuridade em três vezes nas áreas com piores indicadores socioeconômicos, atuando como um modificador de efeito. Erikson et al. (2016), em um estudo no Canadá, relataram que o $\mathrm{MP}_{2.5}$ e o SES no nível da vizinhança estão associados ao menor peso ao nascer, não apenas como fatores independentes, mas também têm um efeito de interação, sugerindo que algumas subpopulações podem ser mais vulneráveis aos efeitos da poluição do 
ar. A modificação da associação entre poluição do $\operatorname{ar}\left(\mathrm{NO}_{2}, \mathrm{CO}\right.$ e $\left.\mathrm{SO}_{2}\right)$ e prematuridade por SES de vizinhança também foi relatada em um estudo australiano (Li S et al., 2016).

No Japão, Yorifuji et al. (2013) reportaram que mulheres com baixo SES tiveram maior risco para BPN em comparação às mães de SES mais alto $(\mathrm{OR}=$ 3,1 IC95\% = 1,2 - 8,2) quando expostas ao mesmo nível de poluição proveniente da via mais próxima. Gray e colaboradores (2014) encontraram, nos EUA, modificação de efeito na exposição materna a $\mathrm{MP}_{2.5}$ e desfechos negativos da gestação devido à renda e educação materna. Foi reportado que, para a mesma exposição a $\mathrm{MP}_{2.5}$, ter 8 anos ou menos de escolaridade foi considerado risco para BPN, PIG e prematuridade (OR = 1,17 IC95\% = 1,08 - 1,26, 1,14 IC95\%= $1,09-1,20$ e 1,04 IC $95 \%=0,99-1,10$ respectivamente), enquanto que 16 ou mais anos de escolaridade teve um efeito protetor para os mesmos desfechos negativos $(\mathrm{OR}=0,72 \mathrm{IC} 95 \%=0,68-0,75,0,72 \mathrm{IC} 95 \%=0,69-0,75$ e 0,78 IC95\% $=0,75-0,80$ respectivamente) assim como baixa renda da moradia também foi apontada como risco para BPN, PIG e prematuridade $(\mathrm{OR}=1,04$ [IC95\% = 1,00 - 1,08], 1,04 [IC95\% = 1,00 - 1,07] e 1,01 [IC95\% = 0,98 - 1,04]) em relação à renda alta $(\mathrm{OR}=0,92$ [IC95\% $=0,88-0,95], 0,93$ [IC95\% $=0,90$ $0,96]$ e 0,90 [IC95\% = 0,87 - 0,93 respectivamente). Yi e colaboradores (2010) encontraram, em um estudo na Coréia do Sul, que um aumento médio de 10 $\mu \mathrm{g} / \mathrm{m}^{3}$ na exposição ao $\mathrm{MP}_{10}$ no segundo trimestre da gestação aumentou a probabilidade de prematuridade em 3,4\% [IC95\% = 0,31\% - 6,58\%] em mães que moravam em áreas com pior SES, enquanto mães que moravam em áreas com indicadores socioeconômicos altos apresentaram uma diminuição na probabilidade de prematuridade de $0,62 \%$ [IC95\% $=-3,74 \%-2,61 \%]$.

Por outro lado, um estudo na Itália relatou que mães com alto nível educacional tinham um risco maior de partos prematuros associado à exposição ao MP10 (Schifano et al., 2013).

A literatura cientifica ainda é bastante controversa no que diz respeito à existência da modificação de efeito do SES tanto na relação exposição a poluição do ar-mortalidade, quanto na relação exposição a poluentes 
atmosféricos-desfechos negativos da gravidez. Em ambos os casos há uma parte significativa dos estudos que indicam a existência de tal modificação de efeito, que mostram que os indivíduos com piores indicadores socioeconômicos são mais susceptíveis e outra parte dos estudos que não encontrou essa maior susceptibilidade. Uma parte pequena dos estudos mostrou que os indivíduos com melhores indicadores socioeconômicos são mais susceptíveis. 


\section{OBJETIVOS}

O objetivo geral deste estudo é examinar o efeito da poluição do ar na saúde da população e avaliar se esse efeito é alterado devido à diferença no nível socioeconômico. Será estudado o município de São Paulo entre os anos de 2011 e 2016.

\subsection{Objetivos específicos}

- Medir o efeito da poluição do ar na mortalidade da população geral e nas faixas etárias mais vulneráveis por todas as causas não externas, por causas respiratórias e cardiovasculares e avaliar se a condição socioeconômica modifica este efeito no município de São Paulo entre 2011 e 2015;

- Medir o efeito da poluição do ar na prematuridade e baixo peso ao nascer em nascidos vivos do município de São Paulo no período entre 2011 e 2016 e verificar se a condição socioeconômica altera esse efeito. 


\title{
4 METODOLOGIA
}

\subsection{Medição da poluição atmosférica}

A resolução do Conselho Nacional do Meio Ambiente (CONAMA) $n \cong 3$, de 28/06/1990 (CONAMA, 1990) considera que poluente atmosférico é

\begin{abstract}
"qualquer forma de matéria ou energia com intensidade e em quantidade, concentração, tempo ou características em desacordo com os níveis estabelecidos, e que tornem ou possam tornar o ar impróprio, nocivo ou ofensivo à saúde, inconveniente ao bem-estar público, danoso aos materiais, à fauna e à flora ou prejudicial à segurança, ao uso e gozo da propriedade e às atividades normais da comunidade."
\end{abstract}

Os poluentes do ar podem ser classificados em primários - quando são emitidos diretamente da fonte, e secundários - quando são formados na atmosfera devido a uma reação química entre o composto ou poluente na atmosfera. Dentre o conjunto de poluentes do ar, os mais frequentemente medidos pelas estações de monitoramento são o material particulado de diâmetros inferiores a $10 \mu \mathrm{m}$ ( $\left.\mathrm{MP}_{10}\right)$, o ozônio $\left(\mathrm{O}_{3}\right)$ e o dióxido de nitrogênio $\left(\mathrm{NO}_{2}\right)$.

$\mathrm{O} \mathrm{MP}_{10}$ constitui-se majoritariamente de material sólido ou líquido que fica suspenso no ar e pode ter forma de poeira, fumaça, fuligem entre outras. Pode ser classificado tanto como poluente primário, quando proveniente de combustão em industrias e veículos automotores, quanto secundário, como resultado de reações de aerossóis na atmosfera (CETESB, 2015).

$\mathrm{O} \mathrm{NO}_{2}$ é classificado como poluente primário e tem a forma de gás com aparência marrom avermelhada. Pode sofrer reações químicas na atmosfera transformando-se em ácido nítrico e outros nitratos (que são parte do $\mathrm{MP}_{10}$ ), além de outros compostos tóxicos. É proveniente de combustão de veículos automotores, processos industriais, usinas térmicas e incinerações (CETESB, 2015). 
$\mathrm{O}_{3}$ é um gás incolor e inodoro, principal componente da névoa fotoquímica. É classificado como poluente secundário resultado da radiação solar nos óxidos de nitrogênio e compostos orgânicos voláteis (CETESB, 2015).

No município de São Paulo, a Companhia Ambiental do Estado de São Paulo (CETESB) é encarregada pelo monitoramento da poluição atmosférica. Entre 2011 e 2016, a CETESB possuía 17 estações automáticas de monitoramento de ar na cidade de São Paulo (Figura 1) nas quais foram medidos $\mathrm{MP}_{10}$ e $\mathrm{NO}_{2}$ em 12 delas e $\mathrm{O}_{3}$ em 14.

O MP10 foi medido nas estações Nossa Senhora do Ó, Marginal Tietê, Pinheiros, Capão Redondo, Congonhas, Santo Amaro, Interlagos, Grajaú, Itaim Paulista, Moóca, Parque Dom Pedro II e Cerqueira César. O dióxido de nitrogênio foi medido nas estações Pico do Jaraguá, Marginal Tietê, Cidade Universitária, Pinheiros, Ibirapuera, Capão Redondo, Congonhas, Interlagos, Grajaú, Itaim Paulista, Parque Dom Pedro II e Cerqueira César. O ozônio foi medido nas estações Nossa Senhora do Ó, Pico do Jaraguá, Cidade Universitária, Pinheiros, Ibirapuera, Capão Redondo, Santo Amaro, Interlagos, Grajaú, Itaquera, Itaim Paulista, Moóca, Parque do Pedro Il e Santana (CETESB, 2018).

No período entre 2011 e 2016 algumas estações de monitoramento foram inauguradas: as estações Capão Redondo e Marginal Tietê iniciaram suas operações em setembro de 2012, Interlagos em fevereiro de 2012 e a estação Itaim Paulista em julho de 2012 (CETESB, 2018).

As estações de monitoramento manuais não fazem as medições de $\mathrm{MP}_{10}$, $\mathrm{O}_{3}$ e $\mathrm{NO}_{2}$ no município de São Paulo. Os dados de $\mathrm{MP}_{10}, \mathrm{O}_{3}$ e $\mathrm{NO}_{2}$ possuem um sistema de validação, os quais são considerados válidos e representativos apenas os dados diários com ao menos 16 das 24 horas sem erros de medição em um dia e a média anual válida se mais da metade das médias diárias de cada quadrimestre (janeiro-abril, maio-agosto e setembro-dezembro) forem válidas (CETESB, 2018). 
Figura 1 - Distribuição das Estações de Monitoramento de Ar do Estado e da Região Metropolitana de São Paulo, CETESB, 2018

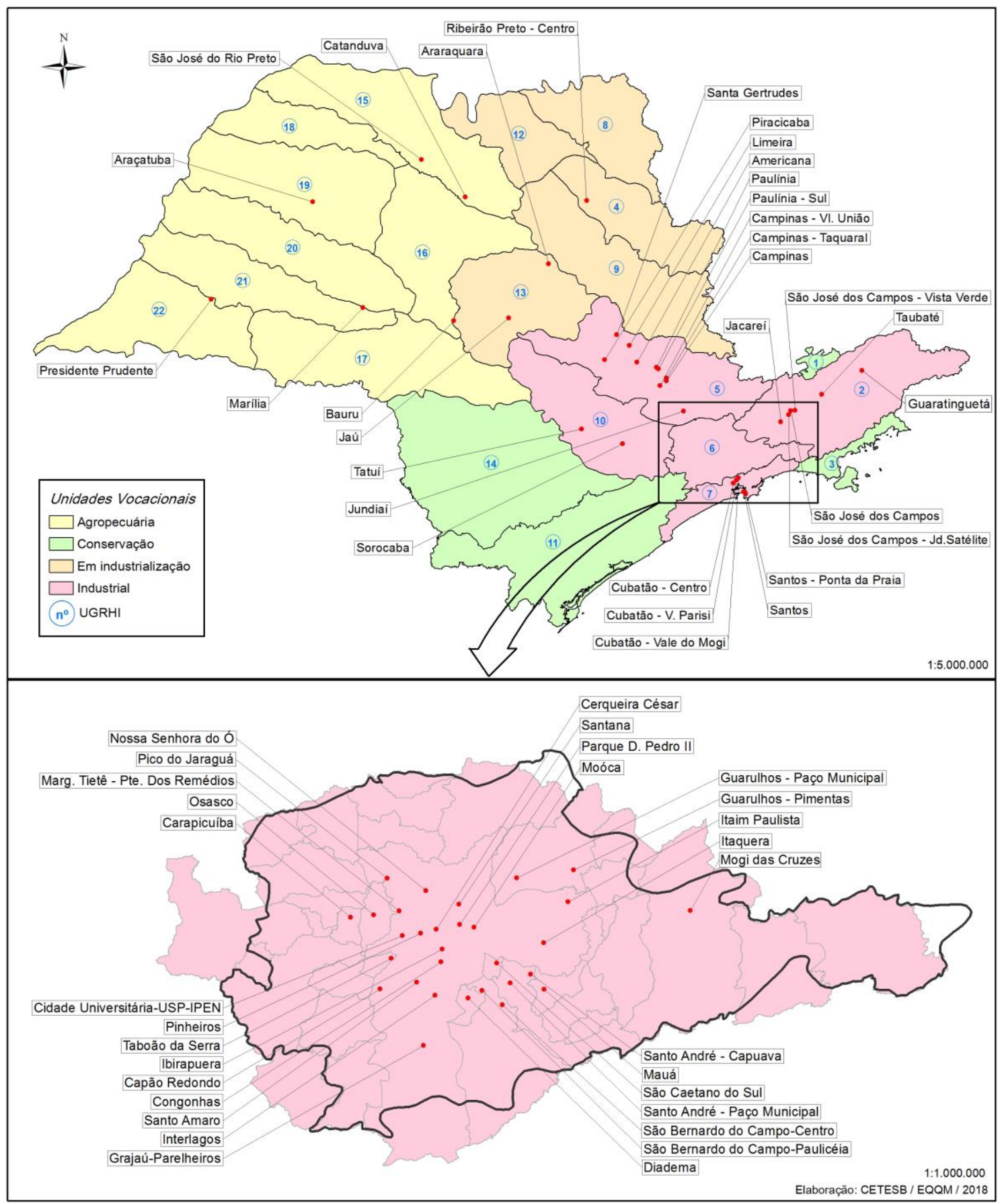

Fonte: CETESB (2018) 


\subsection{Dados de mortalidade}

Até o final do século XIX, cada país possuía um modelo diferente de atestado de óbito. Em 1925, foi publicado um informe sugerindo um modelo único de atestado de óbito para declaração de morte, entretanto apenas em 1948 o Modelo Internacional de Atestado de Óbito foi adotado e passou a ser utilizado por quase todos os países do mundo (CREMERS, 2018).

No Brasil, em 1976, o Ministério da Saúde adotou uma Declaração de Óbito (DO) para todo território nacional. Esta, que ainda mantinha o modelo internacional na parte relativa às causas de morte, uniformizava a maneira de registrar os dados, consolidando-os por meio do Sistema de Informação de Mortalidade (SIM), permitindo melhores análises dos dados coletados (Brasil, 2011). A responsabilidade do preenchimento da Declaração de Óbito é atribuída ao médico, como expresso no artigo 84 do Código de Ética Médica: "é vedado ao médico deixar de atestar óbito de paciente ao qual vinha prestando assistência, exceto quando houver indícios de morte violenta" (CFM, 2009).

A DO tem como objetivo ser o documento padrão para coleta de informação sobre mortalidade subsidiando as estatísticas vitais e epidemiológicas no país, conforme determina o artigo 10 da Portaria no 116, de 11 de fevereiro de 2009 (Brasil, 2009). Essa é padronizada e impressa com uma sequência numérica única, em conjunto de 3 vias. As Secretarias Estaduais de Saúde são responsáveis pela distribuição dos formulários diretamente ou por meio de suas instâncias regionais de saúde (artigo 13 da portaria no 116 MS/SVS de 11/02/2009).

No município de São Paulo a responsabilidade da distribuição dos impressos de DO, alimentação das informações, manutenção do sistema e acesso aos usuários é do PRO-AIM (Programa de Aperfeiçoamento das Informações de Mortalidade) (São Paulo (cidade), c2017).

A DO é composta por 9 blocos e um total de 59 variáveis.

- Bloco I - Identificação. Este bloco com 14 campos se destina a colher informações gerais sobre o falecido e seus pais. Os campos são: tipo de 
óbito (fetal ou não fetal), data do óbito (formato $\mathrm{dd} / \mathrm{mm} / \mathrm{aaaa}$ ), número do cartão do SUS, naturalidade, nome do falecido (no caso de óbito fetal, é preenchido com "Natimorto"), nome do pai (no caso de desconhecido, é preenchido com "Ignorado"), nome da mãe (no caso de desconhecido, é preenchido com "Ignorado"), data de nascimento (no formato $\mathrm{dd} / \mathrm{mm} /$ aaaa, caso óbito fetal, as datas de nascimento e óbito são iguais), idade (em anos; menos que um ano e mais que 29 dias: com o número de meses de vida; entre 1 e 29 dias: com o número de dias; menos de 1 dia: com o número de horas; menos de uma hora: com o número de minutos vividos; óbito fetal: campo não preenchido), sexo (é preenchido como "Ignorado" apenas nos casos especiais como cadáveres mutilados, estado avançado de decomposição ou hermafroditismo), raça (branca, preta, amarela, parda ou indígena - é respondida pela pessoa responsável pelo falecido), situação conjugal (solteiro, casado, viúvo, separado judicialmente/divorciado, união estável ou ignorado), escolaridade (sem escolaridade, fundamental I, fundamental II, médio, superior incompleto ou superior completo), ocupação habitual (de acordo com a CBO).

- Bloco II - Residência. Bloco com 5 campos destinado a colher informações sobre a residência do falecido. Logradouro (rua, praça, avenida, etc. com número e complemento), CEP, bairro/distrito, município de residência e UF.

- Bloco III - Ocorrência. Destinado a colher informações sobre o local onde o óbito ocorreu, possui 7 campos. Local de ocorrência (hospital, outro estabelecimento de saúde, domicílio, via pública, outros ou ignorado), estabelecimento (nome do hospital ou estabelecimento de saúde onde o óbito ocorreu), endereço de ocorrência (caso o óbito tenha ocorrido fora do estabelecimento de saúde ou domicílio), CEP, bairro/distrito, município de ocorrência, UF da ocorrência.

- Bloco IV - Fetal ou menor que 1 ano. Bloco com 10 campos destinado a colher informações sobre a mãe e sobre o falecido nos casos de óbito fetal ou menores de 1 ano. Idade da mãe (em anos), escolaridade (nenhuma, fundamental I, fundamental II, médio, superior incompleto, 
superior completo), ocupação habitual (com código $\mathrm{CBO}$ ), número de filhos (vivos e mortos), número de semanas de gestação (em semanas), tipo da gravidez (única, dupla, tripla ou mais, ignorada), tipo de parto (vaginal, cesáreo ou ignorado), morte em relação ao parto (antes, durante, depois ou ignorado), peso ao nascer (em gramas) e número da DN.

- Bloco V - Condições e causas do óbito. Bloco com 4 campos destinado a qualificar as condições e causas que provocaram o óbito. Contempla o modelo internacional de Atestado de Óbito adotado pela OMS em 1948 e sua importância por ser a fonte da causa básica do óbito. Período do óbito em mulher em idade fértil (na gravidez, no parto, no aborto, até 42 dias após o parto, de 43 a 1 ano após o parto, não ocorreu nesse período ou ignorado), recebeu assistência médica durante a doença que ocasionou a morte (sim, não, ignorado), diagnóstico confirmado por necropsia (sim, não, ignorado), causas da morte (preenchida pelo médico que atestou o óbito com a sigla CID-10 correspondente).

- Bloco VI - Médico. Bloco com 7 campos que colhe informações sobre o médico que assina a DO. Nome do médico, CRM (número do documento do Conselho Regional de Medicina), óbito atestado por médico (assistente, substituto, IML, SVO ou outro), município e UF do SVO ou IML (preenchido apenas quando o campo anterior for preenchido como "SVO"), meio de contato (telefone, e-mail, etc.), data do atestado (formato $\mathrm{dd} / \mathrm{mm} /$ aaaa), assinatura.

- Bloco VII - Causas externas. Bloco com 5 campos que colhe informações sobre o provável tipo de morte por violências. É preenchido apenas quando a causa de morte for referente ao Capítulo XX do CID-10. Tipo de provável circunstância de morte não natural (acidente, suicídio, homicídio, outros ou ignorado), acidente de trabalho (sim, não ou ignorado), fonte da informação (boletim de ocorrência, hospital, família, outra ou ignorado), descrição sumária do evento incluindo o tipo de local da ocorrência, se a ocorrência for em local público anotar endereço logradouro.

- Bloco VIII - Cartório. Bloco com 5 campos destinado a colher dados do Cartório de Registro Civil onde foi efeituado a DO. Cartório (nome do 
cartório por extenso), número do registro, data (formato dd/mm/aaaa), município e UF.

- Bloco IX - Localidade sem médico. Bloco com 2 campos preenchido no caso de óbito ocorrido em localidade sem médico. O registro, nesse caso, fica a cargo do Cartório de Registro Civil. Nome do declarante e assinatura das testemunhas.

Para a realização do presente estudo, foram requeridas as seguintes informações da DO junto ao PRO-AIM, no período entre 2011 e 2015: data do óbito, idade do falecido, sexo, raça, estado civil, escolaridade, distrito de residência e causa básica do óbito. Para a análise de dados sobre mortalidade, foi utilizada a escolaridade como indicador socioeconômico individual.

\subsection{Dados de nascimentos}

Segundo a Organização Mundial de Saúde (OMS), nascimento vivo

"é a expulsão ou extração completa, do corpo da mãe, independente da duração da gestação, de um produto de concepção, o qual, depois da separação, respire ou dê qualquer outro sinal de vida, tal como batimentos do coração, pulsações do cordão umbilical estando ou não desprendida a placenta. Cada produto de um nascimento que reúna essas condições se considera como uma criança viva (OMS, 1995)."

Apesar da existência de fonte de informações sobre nascidos vivos desde a Lei № 6.015 de 31 de dezembro de 1973 (Brasil, 1973), foi apenas a partir da portaria no 649/GM/MS de 04 de julho de 1989 que surgiu o Grupo de Estatísticas Vitais (GEVIMS) para assessorar o Ministério da Saúde a coletar informações sobre mortalidade, natalidade, avaliação de saúde, programas básicos de saúde, planejamento e avaliação em saúde e promoção da utilização de estatísticas vitais (Funasa, 2001).

A partir de então criou-se o Sistema de Informações sobre os Nascidos Vivos (SINASC) e o documento padrão foi denominado de Declaração de 
Nascido Vivo (DN), aprovado em 1990 (Funasa, 2001). O sistema permitiu conhecer e acompanhar variáveis relacionadas à gravidez como peso do recémnascido, idade gestacional e idade materna (Mello Jorge et al., 1993).

A versão de 2011 da DN é dividida em 8 blocos distintos relativos às informações sobre o recém-nascido, a mãe, o pai, local de ocorrência, a gestação e parto, anomalia congênita, preenchimento e cartório.

- Bloco I - Identificação do Recém-nascido: Este bloco destina-se a colher informações referentes ao nascido vivo e tem 6 campos. Nome do recémnascido, data e hora do nascimento (data no formato $\mathrm{dd} / \mathrm{mm} / \mathrm{aaaa}$ ), sexo do recém-nascido, peso ao nascer (em gramas), índice Apgar (varia entre 0 e 10) e detecção de alguma anomalia congênita.

- Bloco II - Local de ocorrência: Esse bloco se destina a colher informações sobre o local onde ocorreu o parto e tem 7 campos. Local de ocorrência (hospital, outro estabelecimento de saúde, domicilio, outros ou ignorado), nome do estabelecimento, endereço do estabelecimento, CEP, bairro/distrito, município de ocorrência e unidade federativa (UF) de ocorrência.

- Bloco III - Mãe: Refere-se aos dados gerais da mãe e da sua história reprodutiva e tem 14 campos. Nome da mãe, número do cartão SUS da mãe, escolaridade da mãe (nenhuma, fundamental $I-1^{\text {a }}$ a $4^{\text {a }}$ série, fundamental II - $5^{\mathrm{a}}$ a $8^{\mathrm{a}}$ série, médio - antigo $2^{\circ}$ grau, superior incompleto, superior completo ou ignorado), ocupação habitual (segundo a Classificação Brasileira de Ocupações (CBO)), data de nascimento (dd/mm/aaaa), idade (em anos), naturalidade, situação conjugal (solteira, casada, viúva, separada judicialmente/divorciada, união estável ou ignorada), raça/cor (branca, preta, amarela, parda, indígena), residência (logradouro, número e complemento), CEP, bairro/distrito, município e UF.

- Bloco IV - Nome e idade do Pai: Bloco com 2 campos, referentes ao nome e idade do pai. Podem ser alterados por decisão judicial ou deixados em branco em caso de dúvida. 
- Bloco V - Gestação e Parto: Bloco destinado a colher informações referentes à gestação e parto. Possui 11 campos. Histórico gestacional (número de gestações anteriores, número de partos vaginais, número de cesáreas, número de nascidos vivos, número de perdas fetais/abortos não inclui a gestação atual), idade gestacional, data da última menstruação (DUM) no formato $\mathrm{dd} / \mathrm{mm} /$ aaaa (caso DUM ignorada, preencher baseado em informações do prontuário utilizando alternativas para estimação da idade gestacional como exame físico ou outros exames (ultrassonografia por exemplo) ou ignorado), número de consultas pré-natal, mês de gestação que iniciou o pré-natal, tipo de gravidez (única, dupla, tripla ou mais ou ignorada), apresentação do parto (cefálica, pélvica ou podática, transversa ou ignorada), se o trabalho de parto foi induzido, tipo de parto (vaginal, cesário ou ignorado), se cesárea começou antes do parto iniciar, e quem deu assistência ao nascimento (médico, enfermeira, obstetriz, parteira, outros ou ignorado).

- Bloco VI-Anomalia Congênita: Bloco com apenas 1 campo caso o campo 6 do bloco 1 for assinalado como "sim". Nesse campo o responsável pelo preenchimento descreve todas as anomalias ou defeitos congênitos observados, sem hierarquia ou tentativa de agrupá-los em síndromes, priorizando a descrição da CID-10.

- Bloco VII - Identificação do responsável pelo preenchimento da DN. Bloco com 6 campos referentes à identificação do responsável pelo preenchimento. Data do preenchimento da DN (dd/mm/aaaa), nome do responsável, função (médico, enfermeira, parteira, funcionário de cartório ou outros), tipo de documento (CNES, CRM, COREN, RG ou CPF), número do documento, órgão emissor do documento.

- Bloco VIII - Cartório. Bloco com 5 campos destinado a informações relativas ao Cartório Civil que assentou o registro do nascimento. Será preenchido com o nome do cartório com código, número do registro, data do registro (dd/mm/aaaa), município e UF.

Para a realização do presente estudo, foram requeridas as seguintes informações da DN junto ao SINASC, no período entre 2011 e 2016: maternas 
(idade da mãe, escolaridade, distrito de residência), gestação e parto (tipo de gestação, número de consultas pré-natais, idade gestacional e tipo de parto), e nascido vivo (data de nascimento, sexo, peso e presença de anomalia congênita). Para as análises de desfechos adversos da gravidez, foi utilizada a educação materna como indicador socioeconômico individual.

\subsection{Dados socioeconômicos contextuais}

Para o município de São Paulo, a Secretaria Municipal de Desenvolvimento Urbano (SMDU) permite o acesso público aos indicadores e informações por meio da página Infocidade (http://infocidade.prefeitura.sp.gov.br). As fontes dos dados são as próprias secretarias da prefeitura, além do IBGE, fundação SEADE e Ministérios do Trabalho e Emprego, da Educação e da Saúde. Nela é possível acessar dados sobre abastecimento alimentar, assistência social, cultura, demografia, economia, educação, esportes e lazer, finanças públicas, habitação, infraestrutura, meio ambiente, mercado de lançamentos imobiliários, saúde, subprefeituras, território, trabalho, transporte e uso do solo urbano.

Para a realização do presente estudo, foram colhidos, para cada um dos 96 distritos do município de São Paulo, as seguintes informações baseadas no censo 2010: renda média; proporção de residências com renda per capita nas categorias, em salários mínimos: até 0,5 , de 0,5 a 1, 1 a 2, 2 a 5, 5 a 10, 10 a 20 e 20 ou mais; taxa de alfabetização; anos de estudo do chefe de família: 0 a 8 , 9 a 11, 12 ou mais; proporção de residências com um morador, 2 moradores, 3 ou 4, 5 ou mais moradores; proporção de residências ligadas ao esgoto; proporção de leitos do Sistema Único de Saúde (SUS) em relação ao total do distrito; proporção de residências ligadas à energia elétrica; proporção de residências ligadas à rede fixa de telefonia e proporção de residências com acesso à telefonia móvel.

Análise de componentes principais com rotação varimax foi realizada utilizando as variáveis coletadas e as variáveis que mais explicaram a 
variabilidade foram: proporção de casas com renda superior a 20 salários mínimos, taxa de alfabetização, residências cujo chefe de família tem 12 ou mais anos de estudo, proporção de residências com pessoas morando sozinhas e proporção de residências não ligadas à rede de esgoto. Após este passo, foram atribuídos pesos a cada uma dessas variáveis proporcionais à variabilidade explicada. Os pesos foram, respectivamente, 23,5\%, 19,5\%, 25\%, 25\% e 7\% e foi então atribuído um escore a cada distrito com base nas variáveis escolhidas. Esse método já foi realizado, com algumas variações, em outros estudos (Blanco-Becerra et al., 2014; Carbajal-Arroyo et al., 2011).

Em seguida, os 96 distritos foram separados em 3 categorias baseadas nos escores calculados e classificados como status socioeconômicos de vizinhança (SESV) alto, médio e baixo (Figura 2). Essa variável foi utilizada como indicador contextual nas análises de mortalidade e de eventos adversos da gravidez.

Foram alocados à categoria alta os distritos: Moema, Jardim Paulista, Itaim Bibi, Alto de Pinheiros, Vila Mariana, Pinheiros, Consolação, Perdizes, Campo Belo, Santo Amaro, Saúde, Morumbi, Vila Leopoldina, Barra Funda, Lapa, Bela Vista, Butantã, Santa Cecília, Liberdade, Santana, Tatuapé, Moóca, Campo Grande, Vila Sônia, Cambuci, Vila Andrade, Socorro, Ipiranga, Cursino, República, Tucuruvi e Belém.

Foram alocados à categoria média os distritos: Água Rasa, Mandaqui, Vila Guilherme, Vila Prudente, Carrão, Rio Pequeno, Jaguaré, Casa Verde, Jabaquara, Vila Formosa, Penha, Bom Retiro, Vila Matilde, Brás, Freguesia do Ó, Jaguará, Pirituba, Limão, São Lucas, Pari, São Domingos, Sacomã, Sé, Artur Alvim, Ponte Rasa, Aricanduva, Vila Maria, Vila Medeiros, Raposo Tavares, Cangaíba, Cidade Dutra e Jaçanã.

Finalmente, foram alocados à categoria mais baixa os distritos: Campo Limpo, José Bonifácio, Cidade Líder, São Miguel, Tremembé, São Mateus, Itaquera, Cachoeirinha, Ermelino Matarazzo, Cidade Ademar, Parque do Carmo, Jardim São Luís, Jaraguá, Vila Jacuí, Capão Redondo, Sapopemba, Cidade Tiradentes, Vila Curuçá, Guaianases, Itaim Paulista, Brasilândia, Perus, 
Pedreira, Anhanguera, Jardim Helena, São Rafael, Lajeado, Iguatemi, Jardim Ângela, Grajaú, Parelheiros e Marsilac. 
Figura 2 - Distribuição espacial dos distritos de São Paulo, segundo status socioeconômico de vizinhança

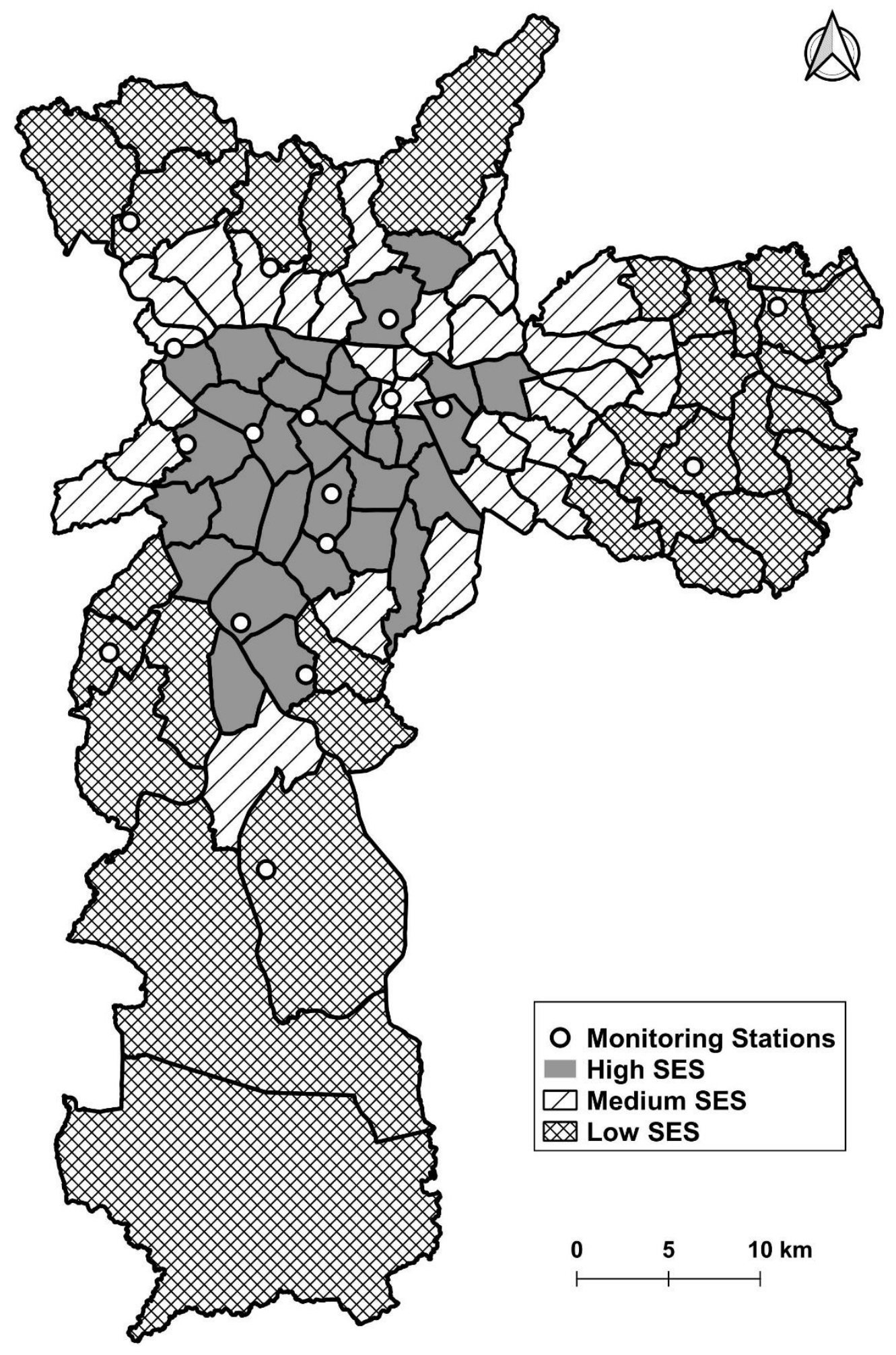




\subsection{Dados meteorológicos}

Nos estudos epidemiológicos envolvendo poluição do ar, a temperatura pode agir como uma variável de confusão, pois ela está associada tanto com a mortalidade e desfechos adversos da gestação quanto com o nível de alguns poluentes como MP10 ou NO 2 (Cesaroni et al., 2013; Gouveia et al., 2000). Sob essa premissa, é necessário ajustar os modelos estatísticos pela temperatura ambiente para remover o viés de confusão que seria inserido no modelo.

Para o presente estudo, dados diários de temperatura e umidade relativa do ar para o período compreendido entre janeiro de 2010 e dezembro de 2016 foram requisitados junto à estação meteorológica do Instituto de Astronomia, Geofísica e Ciências Atmosféricas da Universidade de São Paulo ${ }^{3}$ (USP) localizada no Parque de Ciência e Tecnologia USP.

\subsection{Análise estatística}

\subsubsection{Análise dos dados de mortalidade}

Foi usado um desenho case-crossover em que cada caso atua como seu próprio controle. Utilizou-se uma seleção de controles estratificados no tempo, que permitiu controlar possíveis variáveis de confusão, como idade, dia da semana, sazonalidade e temperatura. Os dias de controle foram selecionados dentro de 1 mês antes da morte, deixando um espaçamento de 7 dias para coincidir com o mesmo dia da semana anterior ao evento $(7,14,21$ e 28 dias antes do evento) e para eliminar a autocorrelação de 6 dias entre todas as observações usadas na análise, bem como a sazonalidade (Levy et al., 2001), o que resultou em 4 controles para cada caso.

Primeiro, estimou-se o risco de mortalidade por mortes não acidentais, cardiovasculares e respiratórias devido à exposição ao $\mathrm{MP}_{10}, \mathrm{NO}_{2} \mathrm{e} \mathrm{O}_{3}$ por meio de uma regressão logística condicional múltipla. Estimou-se o OR para um

\footnotetext{
${ }^{3}$ http://www.estacao.iag.usp.br/contatos.php Acesso em 25/09/2017.
} 
aumento de $10 \mu \mathrm{g} / \mathrm{m}^{3}$ de cada poluente. Também se calculou o aumento percentual do risco de cada desfecho utilizando-se a fórmula (OR -1) multiplicado por 100. Os modelos foram ajustados para o efeito da condição socioeconômica tanto no nível individual, por meio da educação quanto no nível de vizinhança, por meio do SESV, pela temperatura e umidade relativa do ar. Os poluentes entraram nos modelos como variáveis contínuas. Foram testadas várias defasagens (lags) nos efeitos dos poluentes (dia atual da morte (lag0), no dia anterior (lag1) e assim por diante) até 5 dias antes dos eventos. Também foram considerados vários períodos médios cumulativos de defasagens (o lag01 é a média do poluente do dia da morte e 1 dia antes dele, o lag02 é a média dos 3 dias etc.), ajustando-se para a temperatura e umidade relativa do ar.

Finalmente, foram testados se esses efeitos foram modificados pelo SES individual e no nível de vizinhança, introduzindo um termo de interação multiplicativa no modelo, usando a mesma estrutura de defasagem selecionada na etapa anterior, sendo a categoria de SES mais alto (tanto no nível individual quanto de vizinhança) como a referência, ajustando-se para idade, sexo, temperatura e umidade relativa. Os resultados são reportados como aumento percentual do risco de mortalidade devido à educação e SESV, para um aumento de $10 \mathrm{\mu g} / \mathrm{m}^{3}$ do poluente no período caso em relação ao período controle, seguido pelos IC95\% para cada estimativa. Todas as análises estatísticas foram realizadas no software Stata versão $13.0^{4}$.

\subsubsection{Análise dos dados de eventos adversos da gestação}

A exposição materna à poluição do ar em cada trimestre da gravidez foi estimada levando em consideração a duração de cada gestação e a data de nascimento. Para cada nascido vivo foi considerada a exposição média trimestral a cada poluente. Para nascimentos prematuros, a exposição do 3 o trimestre foi calculada a partir da $27^{\mathrm{a}}$ semana de gestação até 0 parto. $O$ mesmo procedimento foi utilizado para o ajuste da temperatura nos modelos.

\footnotetext{
${ }^{4}$ StataCorp, LP 4905 Lakeway Drive, College Station, TX 77845, USA.
} 
Foram realizadas análises de regressão logística multinível univariadas para examinar as associações entre educação materna, SESV, idade materna, temperatura, pré-natal, gênero da criança e tipo de parto com os desfechos prematuridade e BPNT. Categorizou-se a idade materna em menores de 20 anos, 20 a 34 anos e maiores de 34 anos. Os resultados foram reportados como OR.

Para a regressão logística multivariada multinível, foram utilizados modelos multiníveis hierárquicos com efeitos aleatórios para educação e SESV, com intercepto aleatório para cada distrito, para acessar o efeito individual e de vizinhança ajustado para características maternas, do recém-nascido e da gravidez usando a função xtmelogit do software Stata. Os resultados são reportados como OR para um aumento de $10 \mu \mathrm{g} / \mathrm{m}^{3}$ do poluente.

Em seguida, foi testada a interação multiplicativa entre os dois indicadores socioeconômicos com cada poluente e cada desfecho, para acessar a alteração devido à condição socioeconômica na relação desfechos negativos da gravidezpoluição atmosférica, para um aumento de $10 \mu \mathrm{g} / \mathrm{m}^{3}$ de cada poluente, ajustando para as mesmas variáveis dos modelos univariados e utilizando-se o OR como medida de efeito. Em todos os modelos, a categoria mais alta de educação e SESV foram consideradas referências. 


\section{RESULTADOS}

\subsection{Resultados da análise de mortalidade}

De $1^{\circ}$ de janeiro de 2011 a 31 de dezembro de 2015, houve um total de 311.574 óbitos por causas não acidentais no município de São Paulo nos indivíduos acima de 30 anos de idade. Foram excluídos de todas as análises os indivíduos que não possuíam informações sobre o nível educacional nos atestados de óbito (30.889 ou $9,9 \%$ do total). Como todas as outras variáveis estudadas não possuíam dados ausentes, a população final de estudo foi de 280.685 indivíduos. Os indivíduos tinham, na maioria, entre 75 e 109 anos $(47,1 \%)$, entre 0 e 8 anos de estudo $(70,9 \%)$ e moravam em distritos mais desfavorecidos $(38,4 \%)$. Proporções semelhantes foram observadas quando examinando especificamente os desfechos cardiovasculares e respiratórios (tabela 1). A idade média para todas as causas não acidentais foi de 71,1 anos $( \pm 15,3)$, semelhante aos desfechos cardiovasculares e respiratórios. As mulheres tinham, em média, 5,7 anos mais que os homens e menos escolaridade (12,6\% possuíam 12 anos ou mais de estudo versus 7,9\%). Havia mais mulheres do que homens que viviam em distritos com melhores indicadores socioeconômicos (29,9\% versus $25,7 \%)$. A média de idade no grupo de baixa escolaridade foi de 72,8 anos, enquanto no ensino médio foi de 68,7 anos. A idade média dos indivíduos que vivem em distritos com baixo nível socioeconômico foi de 66,7 anos, enquanto nos distritos com alto índice foi de 76,4 anos. 
Tabela 1: Estatísticas descritivas da mortalidade da população de estudo, por causa de morte, São Paulo, 2011 - 2015

$$
\text { Não-acidental N (\%) Cardiovascular N (\%) Respiratória N (\%) }
$$

\begin{tabular}{|c|c|c|c|}
\hline Total & 280685 & 102032 & 40973 \\
\hline \multicolumn{4}{|l|}{ Grupo etário } \\
\hline $30-64$ & $91859(32,7)$ & $31038(30,4)$ & $8780(21,5)$ \\
\hline $65-74$ & $56701(20,2)$ & $21388(21,0)$ & $7254(17,8)$ \\
\hline $75-109$ & $132125(47,1)$ & $49606(48,6)$ & $24759(60,7)$ \\
\hline \multicolumn{4}{|l|}{ Gênero } \\
\hline Masculino & $140594(50,1)$ & $51200(50,2)$ & $20716(50,8)$ \\
\hline Feminino & $140091(49,9)$ & $50832(49,8)$ & $20077(49,2)$ \\
\hline \multicolumn{4}{|l|}{ Educação } \\
\hline 0 a 8 anos & $199086(70,9)$ & $75027(73,5)$ & $31032(76,1)$ \\
\hline 9 a 11 anos & $52755(18,8)$ & $18503(18,1)$ & $6408(15,7)$ \\
\hline 12 ou mais anos & $28844(10,3)$ & $8502(8,4)$ & $3353(8,2)$ \\
\hline \multicolumn{4}{|l|}{ SESV } \\
\hline Baixa & $107737(38,4)$ & $41994(41,2)$ & $15137(37,1)$ \\
\hline Média & $94851(33,8)$ & $34607(33,9)$ & $13810(33,9)$ \\
\hline Alta & $78097(27,8)$ & $25431(24,9)$ & $11846(29,0)$ \\
\hline
\end{tabular}

Durante o período analisado, o valor médio diário de $\mathrm{MP}_{10}$ foi $38,7 \mu \mathrm{g} / \mathrm{m}^{3}$ $\left( \pm 16,0 \mu \mathrm{g} / \mathrm{m}^{3}\right), \circ \mathrm{NO}_{2}$ foi $78,9 \mu \mathrm{g} / \mathrm{m}^{3}\left( \pm 33,5 \mu \mathrm{g} / \mathrm{m}^{3}\right)$ e a média das médias móveis diárias de $\mathrm{O}_{3}$ foi $69,4 \mu \mathrm{g} / \mathrm{m}^{3}\left( \pm 32,0 \mu \mathrm{g} / \mathrm{m}^{3}\right)$, a temperatura média diária foi de cerca de $20{ }^{\circ} \mathrm{C}$ e a umidade relativa de $80 \%$ (tabela 2). A correlação entre poluentes foi alta para $\mathrm{MP}_{10} \mathrm{e} \mathrm{NO}_{2}$ ( $\mathrm{r}$ de Pearson $=0,750$ ), mas não tão alta para 
os outros poluentes. $\mathrm{O}_{3}$ teve uma correlação média com a temperatura e correlação negativa com a umidade relativa do ar (Tabela 3).

Tabela 2: Medidas descritivas dos poluentes e medições meteorológicas, São Paulo, $2011-2015$

\begin{tabular}{lccccccc} 
& Média & DP & Min & 25 th & 50 th & 75 th & Max \\
& & & & & & & \\
\hline $\mathrm{O}_{3}\left(\mu \mathrm{g} / \mathrm{m}^{3}\right)$ & 69,4 & 32,0 & 3,7 & 45,0 & 65,3 & 90,7 & 187,0 \\
$\mathrm{MP}_{10}\left(\mu \mathrm{g} / \mathrm{m}^{3}\right)$ & 38,7 & 16,0 & 9,5 & 27,0 & 35,7 & 46,7 & 110,7 \\
$\mathrm{NO}_{2}\left(\mu \mathrm{g} / \mathrm{m}^{3}\right)$ & 78,9 & 33,5 & 11,0 & 54,0 & 74,0 & 100,0 & 209,0 \\
Temperatura $\left({ }^{\circ} \mathrm{C}\right)$ & 19,7 & 3,3 & 7,3 & 17,5 & 19,8 & 22,2 & 28,0 \\
Umidade Relativa (\%) & 79,8 & 8,9 & 41,2 & 74,9 & 80,8 & 86,0 & 96,9 \\
\hline
\end{tabular}

Tabela 3: Correlação de Pearson entre os poluentes e parâmetros meteorológicos, São Paulo, 2011 - 2015

\begin{tabular}{lcccc} 
& $\mathrm{O}_{3}$ & $\mathrm{MP}_{10}$ & $\mathrm{NO}_{2}$ & Temperatura \\
\hline $\mathrm{MP}_{10}$ & 0,339 & & & \\
$\mathrm{NO}_{2}$ & 0,422 & 0,750 & & \\
Temperatura & 0,593 & 0,031 & 0,189 & \\
Umidade Relativa & $-0,600$ & $-0,551$ & $-0,495$ & $-0,384$ \\
\hline
\end{tabular}

Foram estimados os efeitos principais dos poluentes na mortalidade não acidental, cardiovascular e respiratória de adultos no município de São Paulo, ajustando-se por idade, sexo, temperatura e umidade relativa. Foram testados vários dias de defasagem ( 0 a 5) e valores médios entre as defasagens (lag01, lag02, até lag05) e foram selecionados os modelos com base nos menores valores de máxima-verossimilhança. Foram encontradas associações positivas entre um aumento de $10 \mu \mathrm{g} / \mathrm{m}^{3}$ nos níveis de $\mathrm{O}_{3}$ e $\mathrm{NO}_{2}$ e risco de mortalidade para todos os desfechos, mas não para a exposição ao $\mathrm{MP}_{10}$ (tabela 4). Encontrou-se uma modificação de efeito estatisticamente significativa da 
educação sobre $\circ \mathrm{O}_{3}$ na categoria de 9 a 11 anos para causas não acidentais de morte e uma interação cruzada na categoria de 0 a 8 anos para exposição à $\mathrm{MP}_{10}$ nos desfechos não acidentais e cardiovasculares. Também foi encontrada uma modificação de efeito estatisticamente significativa do SESV nas categorias baixa e média pela exposição a $\mathrm{NO}_{2}$ em mortes não acidentais. Nos desfechos cardiovasculares, a modificação de efeito ocorreu nos distritos de SESV mais baixos devido à exposição ao $\mathrm{NO}_{2}$. Já para a mortalidade por causas respiratórias, a modificação de efeito ocorreu nos distritos pertencentes a categoria média do SESV devido à exposição ao $\mathrm{MP}_{10}$ e $\mathrm{NO}_{2}$ (tabelas 5 a 7 ).

Tabela 4: Mudança percentual no risco de mortalidade associada a um aumento de $10 \mu \mathrm{g} / \mathrm{m}^{3}$ na concentração dos poluentes com IC95\% para cada desfecho São Paulo, 2011-2015

\begin{tabular}{lccc} 
& $\mathrm{O}_{3}(\operatorname{lag} 1)$ & $\mathrm{MP}_{10}(\operatorname{lag} 1)$ & $\mathrm{NO}_{2}(\operatorname{lag} 0)$ \\
\hline Não acidental & $0,73(0,69 ; 0,78)^{*}$ & $0,77(-0,29 ; 1,73)$ & $1,37(1,33 ; 1,42)^{*}$ \\
Cardiovascular & $0,77(0,68 ; 0,85)^{*}$ & $0,97(-0,76 ; 2,70)$ & $1,34(1,25 ; 1,42)^{*}$ \\
Respiratória & $0,69(0,56 ; 0,83)^{*}$ & $1,28(-1,45 ; 4,02)$ & $1,32(1,19 ; 1,45)^{*}$ \\
\hline & & &
\end{tabular}


Tabela 5: Alteração percentual do efeito da poluição do ar na mortalidade por causas não acidentais devido à educação e SESV associada a um aumento de $10 \mu \mathrm{g} / \mathrm{m}^{3}$ nos poluentes, com IC95\%, São Paulo, 2011-2015

$$
\mathrm{O}_{3}(\operatorname{lag} 1) \quad \mathrm{MP}_{10}(\operatorname{lag} 1) \quad \mathrm{NO}_{2}(\operatorname{lag} 0)
$$

\begin{tabular}{llll}
\hline Educação & & & \\
0 a 8 anos & $0,36(-0,12 ; 0,84)$ & $1,29(0,34 ; 2,25)^{*}$ & $-0,49(-0,98 ;-0,06)$ \\
9 a 11 anos & $0,59(0,05 ; 1,13)^{*}$ & $0,81(-0,26 ; 1,88)$ & $-0,28(-0,82 ; 0,27)$ \\
12 ou mais anos & 0 & 0 & 0 \\
SESV & & \\
Baixo & $0,18(-0,18 ; 0,54)$ & $-0,15(-0,86 ; 0,56)$ & $0,63(0,27 ; 0,99)^{*}$ \\
Médio & $0,36(-0,04 ; 0,72)$ & $0,12(-0,60 ; 0,83)$ & $0,60(0,23 ; 0,97)^{*}$ \\
Alto & 0 & 0 & 0 \\
\hline * estatisticamente significante $($ valor de $p<0,05)$ & &
\end{tabular}

Tabela 6: Alteração percentual do efeito da poluição do ar na mortalidade por causas cardiovasculares devido à educação e SESV associada a um aumento de $10 \mu \mathrm{g} / \mathrm{m}^{3}$ nos poluentes, com IC95\%, São Paulo, 2011-2015

$$
\mathrm{O}_{3}(\operatorname{lag} 1) \quad \mathrm{MP}_{10}(\operatorname{lag} 1) \quad \mathrm{NO}_{2}(\operatorname{lag} 0)
$$

\begin{tabular}{|c|c|c|c|}
\hline \multicolumn{4}{|l|}{ Educação } \\
\hline 0 a 8 anos & $0,50(-0,37 ; 1,37)$ & $2,03(0,29 ; 3,76)^{*}$ & $-0,09(-0,98 ; 0,78)$ \\
\hline 9 a 11 anos & $0,61(-0,36 ; 1,57)$ & $1,29(-0,62 ; 3,20)$ & $-0,43(-1,40 ; 0,54)$ \\
\hline 12 ou mais anos & 0 & 0 & 0 \\
\hline \multicolumn{4}{|l|}{ SESV } \\
\hline Baixo & $0,12(-0,48 ; 0,73)$ & $-0,62(-1,81 ; 0,56)$ & $0,68(0,06 ; 1,29)^{*}$ \\
\hline Médio & $0,26(-0,35 ; 0,88)$ & $-0,48(-1,69 ; 0,73)$ & $0,30(-0,33 ; 0,92)$ \\
\hline Alto & 0 & 0 & 0 \\
\hline
\end{tabular}


Tabela 7: Alteração percentual do efeito da poluição do ar na mortalidade por causas respiratórias devido à educação e SESV associada a um aumento de 10 $\mu \mathrm{g} / \mathrm{m}^{3}$ nos poluentes, com IC95\%, São Paulo, 2011-2015

\begin{tabular}{|c|c|c|c|}
\hline & $\mathrm{O}_{3}$ (lag 1) & $\mathrm{MP}_{10}$ (lag 1) & $\mathrm{NO}_{2}$ (lag 0) \\
\hline \multicolumn{4}{|l|}{ Educação } \\
\hline 0 a 8 anos & $0,55(-0,85 ; 1,95)$ & $-1,53(-4,20 ; 1,14)$ & $-0,44(-1,84 ; 0,96)$ \\
\hline 9 a 11 anos & $0,98(-0,59 ; 2,56)$ & $-1,16(-4,18 ; 1,86)$ & $0,83(-0,74 ; 2,41)$ \\
\hline 12 ou mais anos & 0 & 0 & 0 \\
\hline \multicolumn{4}{|l|}{ SESV } \\
\hline Baixo & $0,17(-0,77 ; 1,11)$ & $1,49(-0,33 ; 3,32)$ & $0,58(-0,36 ; 1,53)$ \\
\hline Médio & $0,48(-0,46 ; 1,43)$ & $2,80(0,98 ; 4,64)^{*}$ & $1,70(0,75 ; 2,65)^{*}$ \\
\hline Alto & 0 & 0 & 0 \\
\hline
\end{tabular}

\subsection{Resultados da análise dos desfechos adversos na gestação}

Entre 2011 e 2016, houve 1.042.923 nascidos vivos no município de São Paulo. Foram excluídas todas as gestações múltiplas (27.420) e aquelas cujos partos tiveram menos de $500 \mathrm{~g}$ ou mais de $5.500 \mathrm{~g}$ (96). Considerou-se que os nascidos vivos nessas condições seriam produtos de gestações de alto risco e o efeito da poluição do ar seria superado por outras comorbidades. Da mesma forma foram excluídos os partos com idade gestacional superior a 42 semanas (13.867) e todos os nascimentos que apresentavam anomalias congênitas (17.370). Por fim, foram removidos todos os nascimentos que tinham dados ausentes em pelo menos uma das covariáveis (4.869). A amostra final incluiu 979.309 nascimentos para análise de prematuridade e 888.133 para análise BPNT.

No geral, a prevalência de prematuridade foi de $9,3 \%$ e de $3,5 \%$ para BPNT nessa população. As mães tinham em sua maioria 20 a 34 anos $(69,6 \%)$, possuíam 9 a 11 anos de escolaridade (61\%), moravam em distritos menos 
favorecidos $(55,4 \%)$ e tinham 4 ou mais atendimentos durante o pré-natal $(94,3 \%)$. Observou-se que a prevalência de prematuros foi maior em recémnascidos do sexo masculino, enquanto a BPNT foi maior no sexo feminino. Mães mais jovens e mais idosas tiveram maior probabilidade de ter ambos os resultados adversos ao nascimento. Os indicadores SES mostraram que tanto as mães com baixa e média educação, quanto as que moram nos distritos de baixo/médio SESV tinham maior probabilidade de apresentar prematuridade e BPNT. Os partos cesáreos foram responsáveis por mais da metade dos partos deste estudo e foram distribuídos de forma desigual de acordo com a educação materna ( $34 \%$ baixo versus $78 \%$ no alto) e SESV ( $46 \%$ no baixo versus $66 \%$ no alto) e demonstrou ser associado à prematuridade mas não ao BPNT. Como esperado, o pré-natal teve um grande efeito em ambos os desfechos, pois as mães que tiveram menos de quatro atendimentos tiveram maior probabilidade de ter desfechos adversos ao nascimento. A temperatura no terceiro trimestre foi positivamente associada à prematuridade. Estatísticas descritivas completas para essas características são mostradas na Tabela 8, bem como os OR das regressões logísticas multiníveis univariadas. 
Tabela 8: Estatísticas descritivas e OR com IC95\% provenientes da regressão logística multinível univariada da associação entre desfechos adversos da gravidez e covariáveis para a população de estudo, São Paulo, 2011-2016

\begin{tabular}{|c|c|c|c|c|c|c|}
\hline Variáveis & Total $n=979306$ & $\begin{array}{c}\text { Nascidos } \\
\text { prematuros } \mathrm{n}(\%)\end{array}$ & $\begin{array}{l}\text { Odds Ratio } \\
\text { (IC95\%) }^{a}\end{array}$ & $\begin{array}{c}\text { Nascidos a termo } \\
\mathrm{n}=888133\end{array}$ & BPNT n(\%) & $\begin{array}{l}\text { Odds Ratio } \\
\left(\text { IC95\%) }^{a}\right.\end{array}$ \\
\hline \multicolumn{7}{|l|}{ Gênero } \\
\hline Feminino $^{b}$ & $478635(48,8 \%)$ & $42528(8,9)$ & \multirow{2}{*}{$\begin{array}{c}1,00 \\
1,10(1,09 ; 1,11)\end{array}$} & $436107(49,1 \%)$ & $18506(4,2)$ & \multirow{2}{*}{$\begin{array}{c}1,00 \\
0,67(0,65 ; 0,69)\end{array}$} \\
\hline Masculino & $500671(51,2 \%)$ & $48645(9,7)$ & & $452026(50,9 \%)$ & $13080(2,9)$ & \\
\hline \multicolumn{7}{|l|}{ Idade materna } \\
\hline$<20$ anos & $129461(13,2 \%)$ & $14429(11,1)$ & $1,32(1,29 ; 1,35)$ & $115032(23,0 \%)$ & $5012(4,4)$ & \multirow{3}{*}{$\begin{array}{c}1,00 \\
1,11(1,07 ; 1,14)\end{array}$} \\
\hline 20 a 34 anos $^{b}$ & $682131(69,6 \%)$ & $59127(8,7)$ & \multirow{2}{*}{$\begin{array}{c}1,00 \\
1,24(1,21 ; 1,26)\end{array}$} & $623004(70,1 \%)$ & $20988(3,4)$ & \\
\hline$>34$ anos & $167714(17,2 \%)$ & $17617(10,5)$ & & 150097 (16,9\%) & $5586(3,7)$ & \\
\hline \multicolumn{7}{|l|}{ Educação materna } \\
\hline $12+$ anos $^{b}$ & $260412(26,6 \%)$ & $22973(8,8)$ & \multirow{2}{*}{$\begin{array}{c}1,00 \\
1,06(1,04 ; 1,08)\end{array}$} & $237439(26,7 \%)$ & $6766(2,8)$ & \multirow{3}{*}{$\begin{array}{c}1,00 \\
1,28(1,24 ; 1,31) \\
1,73(1,67 ; 1,80)\end{array}$} \\
\hline 9 a 11 anos & $597716(61,0 \%)$ & $55734(9,3)$ & & $541982(61,0 \%)$ & $19554(3,6)$ & \\
\hline 0 to 8 anos & $121178(22,4 \%)$ & $12466(10,3)$ & $1,18(1,16 ; 1,21)$ & $108712(12,3 \%)$ & $5266(4,8)$ & \\
\hline \multicolumn{7}{|l|}{ SESV } \\
\hline Alto b & $168955(17,3 \%)$ & $15014(8,9)$ & \multirow{3}{*}{$\begin{array}{c}1,00 \\
1,07(1,04 ; 1,09) \\
1,06(1,04 ; 1,08)\end{array}$} & 153941 (17,3\%) & $4507(2,9)$ & \multirow{3}{*}{$\begin{array}{c}1,00 \\
1,16(1,12 ; 1,21) \\
1,32(1,28 ; 1,37)\end{array}$} \\
\hline Médio & 267649 (27,3\%) & $25274(9,4)$ & & $242375(27,3 \%)$ & $8218(3,4)$ & \\
\hline Baixo & $542702(55,4 \%)$ & $50885(9,4)$ & & $491817(55,4 \%)$ & $18861(3,8)$ & \\
\hline \multicolumn{7}{|l|}{ Tipo de Parto } \\
\hline Vaginal $^{b}$ & $461010(47,1 \%)$ & $41905(9,1)$ & \multirow{2}{*}{$\begin{array}{c}1,00 \\
1,05(1,03 ; 1,06)\end{array}$} & $419105(47,2 \%)$ & $15167(3,6)$ & \multirow{2}{*}{$\begin{array}{c}1,00 \\
0,97(0,94 ; 0,99)\end{array}$} \\
\hline Cesáreo & $518296(52,9 \%)$ & $49268(9,5)$ & & $469028(52,8 \%)$ & $16419(3,5)$ & \\
\hline \multicolumn{7}{|l|}{ Consultas pré-natais } \\
\hline 4 ou mais ${ }^{b}$ & $923429(94,3 \%)$ & $78252(8,5)$ & \multirow{3}{*}{$\begin{array}{c}1,00 \\
3,23(3,16 ; 3,31) \\
3,30(3,17 ; 3,44)\end{array}$} & 845177 (95,2\%) & $28431(3,4)$ & 1,00 \\
\hline 1 a 3 & 43224 (4,4\%) & $9957(23,0)$ & & $33267(3,7 \%)$ & $2148(6,5)$ & \multirow{2}{*}{$\begin{array}{l}1,98(1,89 ; 2,07) \\
3,33(3,12 ; 3,56)\end{array}$} \\
\hline Nenhuma & $12653(1,3 \%)$ & $2964(23,4)$ & & $9689(1,1 \%)$ & $1007(10,4)$ & \\
\hline
\end{tabular}

a Odds Ratio da regressão logística multinível univariada

b Categoria de referência 
A análise de regressão logística multinível avaliou o efeito da exposição aos poluentes durante todo o período gestacional e em cada trimestre separadamente, ajustando-se por sexo da criança, idade materna, escolaridade materna, SESV, tipo de parto, pré-natal e temperatura média no período. Para um aumento de $10 \mu \mathrm{g} / \mathrm{m}^{3}$ nos níveis médios de $\mathrm{MP}_{10}$ no $1^{\circ}$, $2^{\circ}$ e $3^{\circ}$ trimestres e em toda a gravidez, encontrou-se um aumento estatisticamente significativo no risco de BPNT. Níveis mais altos de $\mathrm{NO}_{2}$ durante $01^{\circ}$ e $02^{\circ}$ trimestres e toda a gravidez também foram associados positivamente ao BPNT. O risco de prematuridade foi associado a um aumento de $10 \mu \mathrm{g} / \mathrm{m}^{3}$ nos níveis de $\mathrm{O}_{3}$ durante o segundo e o terceiro trimestres e toda a gravidez e também com os níveis de $\mathrm{MP}_{10}$ no $1^{\circ}$ trimestre e na gravidez inteira (Tabela 9 ).

Tabela 9: Odds Ratios (IC95\%) dos modelos de regressão logística multinível, ajustados por covariáveis, São Paulo, 2011-2016

\begin{tabular}{|c|c|c|c|c|c|}
\hline Desfecho & Poluente & 1ํ Trimestre & $2^{0}$ Trimestre & 3ㅇ Trimestre & Toda gestação \\
\hline & $\mathrm{O}_{3}$ & $0,99(0,98 ; 1,00)$ & $1,06(1,05 ; 1,06)^{*}$ & $1,03(1,02 ; 1,04)^{*}$ & $1,14(1,13 ; 1,16)^{\star}$ \\
\hline \multirow[t]{3}{*}{ Premat } & $\mathrm{NO}_{2}$ & $0,98(0,97 ; 0,99)$ & $0,97(0,96 ; 0,98)$ & $1,07(0,93 ; 1,14)$ & $1,02(0,98 ; 1,05)$ \\
\hline & $\mathrm{MP}_{10}$ & $1,02(1,01 ; 1,03)^{*}$ & $1,01(1,00 ; 1,02)^{*}$ & $0,98(0,95 ; 1,01)$ & $1,08(1,02 ; 1,15)^{\star}$ \\
\hline & $\mathrm{O}_{3}$ & $0,99(0,98 ; 1,00)$ & $1,00(0,99 ; 1,01)$ & $1,01(1,00 ; 1,02)^{*}$ & $1,00(0,98 ; 1,02)$ \\
\hline \multirow[t]{2}{*}{ BPNT } & $\mathrm{NO}_{2}$ & $1,01(1,00 ; 1,02)^{*}$ & $1,01(1,00 ; 1,02)^{*}$ & $1,00(0,99 ; 1,01)$ & $1,01(1,00 ; 1,02)^{*}$ \\
\hline & $\mathrm{MP}_{10}$ & $1,03(1,01 ; 1,07)^{\star}$ & $1,02(1,01 ; 1,04)^{*}$ & $1,04(1,01 ; 1,07)^{*}$ & $1,08(1,03 ; 1,14)^{\star}$ \\
\hline
\end{tabular}

Ao avaliar a modificação do efeito pelo SES, concluiu-se que a menor escolaridade materna leva a um risco adicional de prematuridade e BPNT para todos os poluentes e em todos os trimestres e para toda a gestação. Mães com 9 a 11 anos de estudo também apresentaram maior risco de prematuridade em comparação às mães com 12 anos ou mais, associadas à exposição ao $\mathrm{O}_{3}$ no $3^{\circ}$ trimestre e com $\mathrm{MP}_{10} \mathrm{em}$ todos os períodos. Em relação ao SESV, as mães que vivem em distritos das categorias baixas e médias apresentaram maior risco de BPNT associado à exposição a todos os poluentes em todos os períodos, 
enquanto a modificação do efeito não foi consistente para prematuridade (Gráficos 1 a 4).

Grafico 1: Modificação de efeito da educação materna no risco de prematuridade associado a um aumento de $10 \mu \mathrm{g} / \mathrm{m}^{3}$ de cada poluente para todos os trimestres e todo o período gestacional, São Paulo, 2011-2016

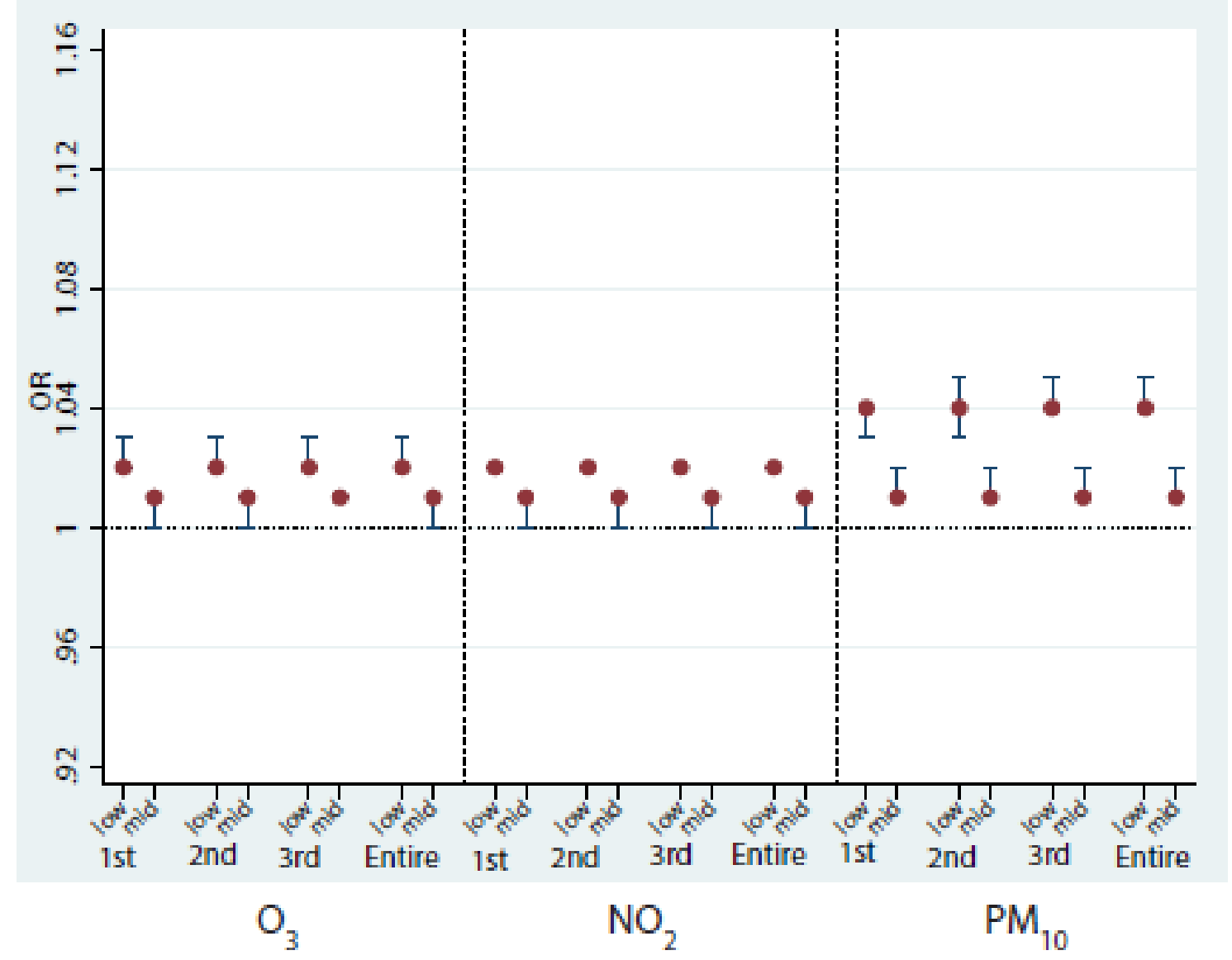


Grafico 2: Modificação de efeito do SESV no risco de prematuridade associado a um aumento de $10 \mu \mathrm{g} / \mathrm{m}^{3}$ de cada poluente para todos os trimestres e todo 0 período gestacional, São Paulo, 2011-2016

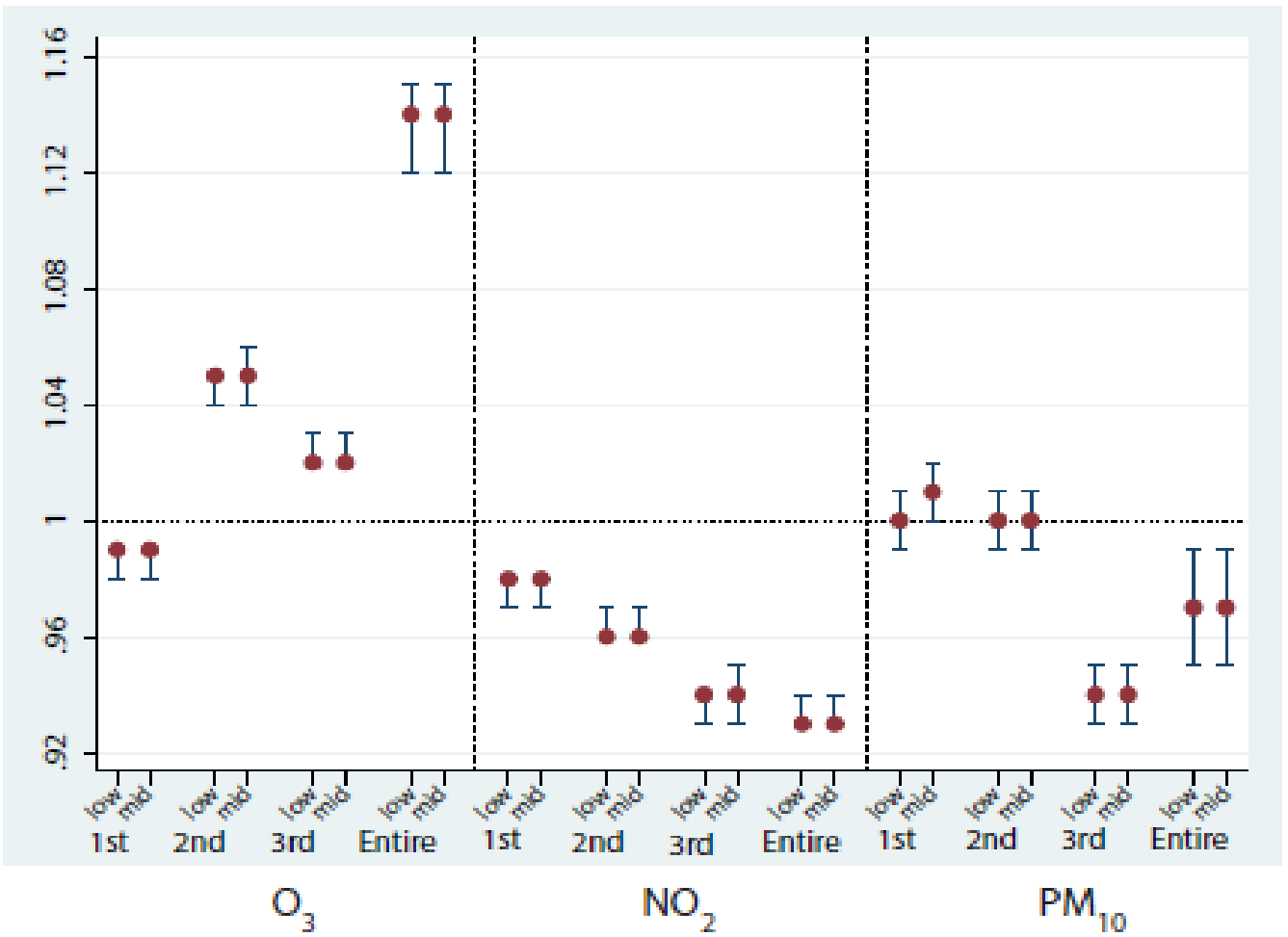

Gráfico 3: Modificação de efeito da educação materna no risco de BPNT associado a um aumento de $10 \mu \mathrm{g} / \mathrm{m}^{3}$ de cada poluente para todos os trimestres e todo o período gestacional, São Paulo, 2011-2016

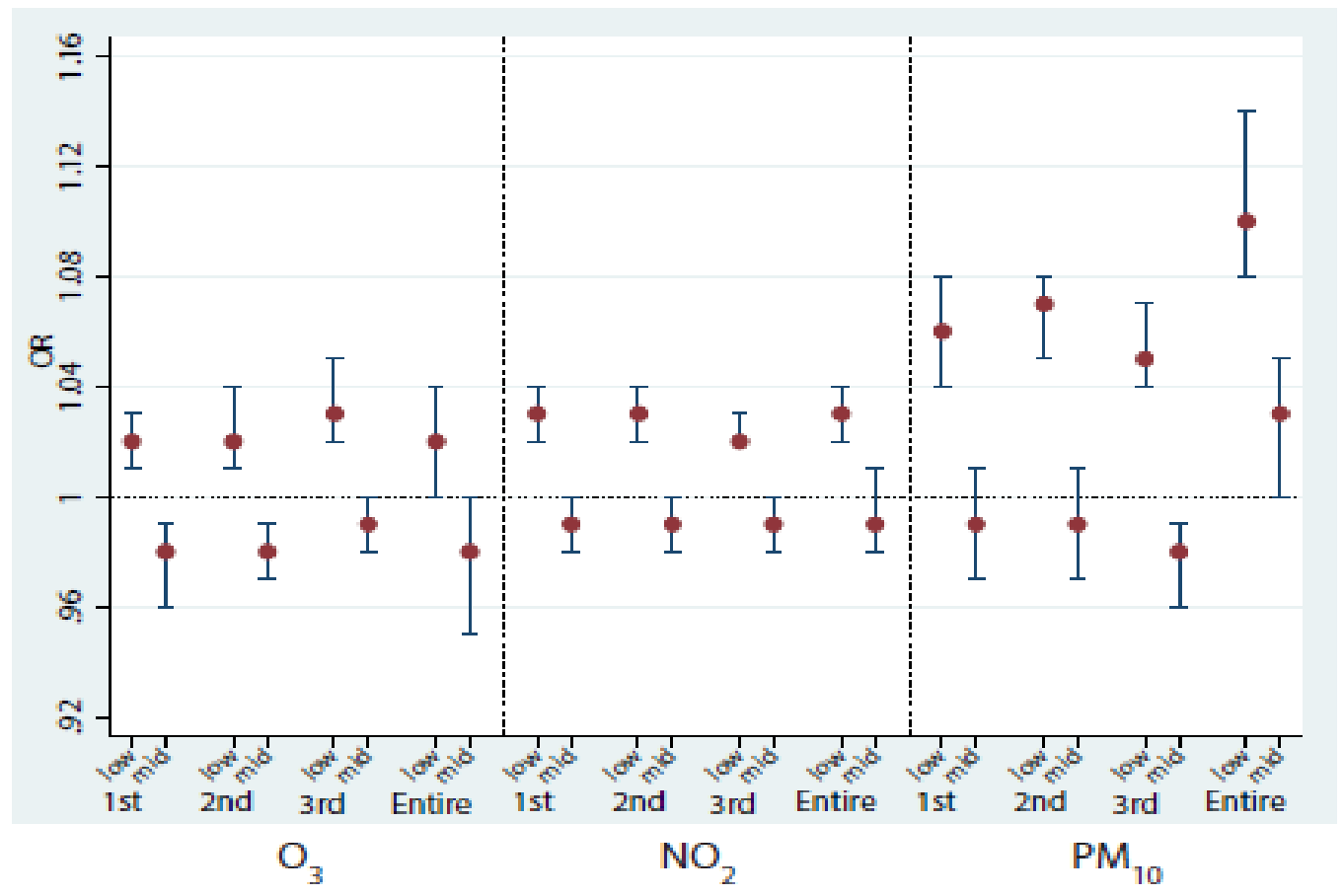


Gráfico 4: Modificação de efeito do SESV no risco de BPNT associado a um aumento de $10 \mu \mathrm{g} / \mathrm{m}^{3}$ de cada poluente para todos os trimestres e todo $O$ período gestacional, São Paulo, 2011-2016

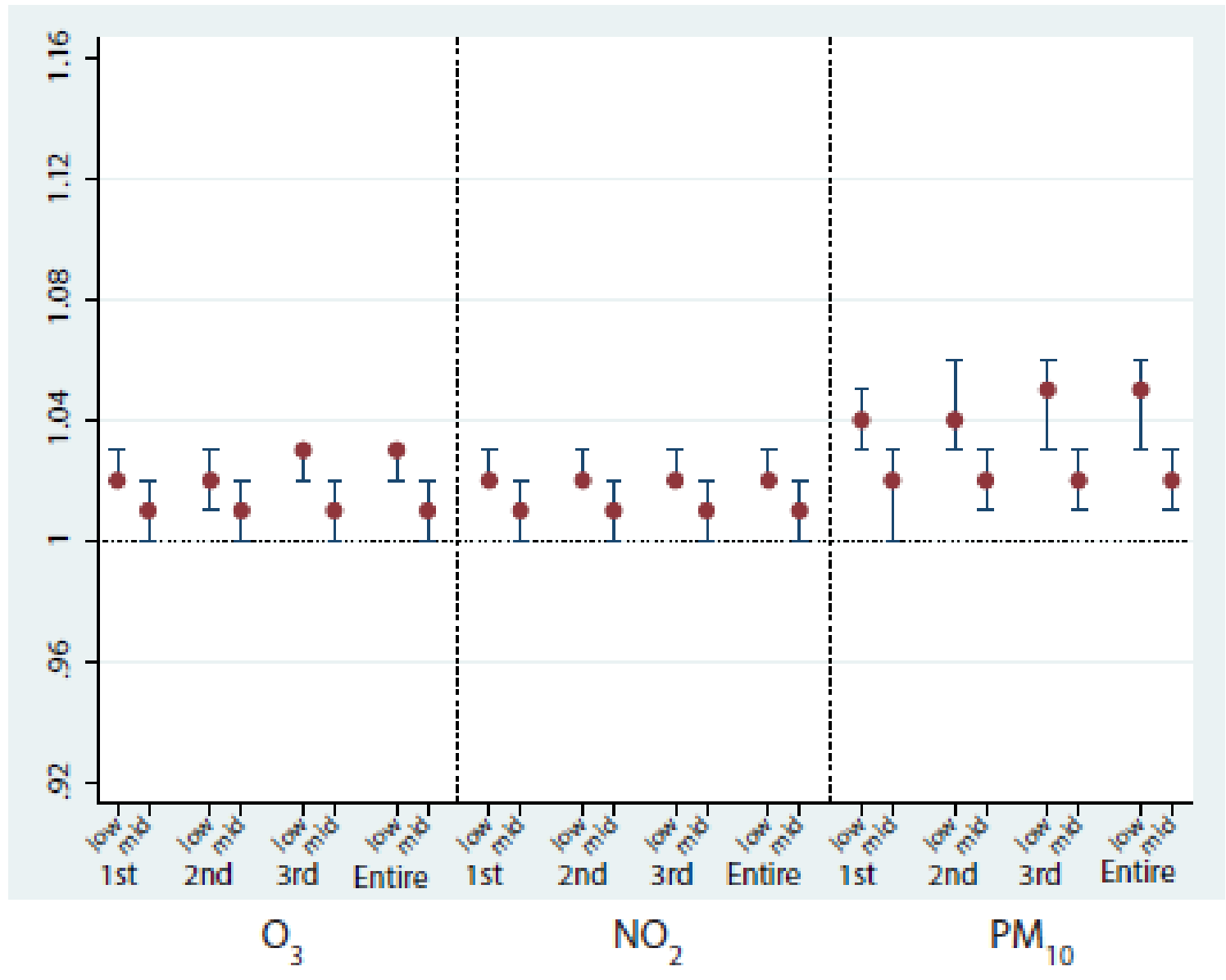




\section{DISCUSSÃO}

Neste estudo, foi avaliado se o status socioeconômico em nível individual e de vizinhança modifica a associação entre poluição do ar e mortalidade por causas não acidentais, cardiovasculares, respiratórias, prematuridade em recém-nascidos e BPNT. Os resultados indicaram modificação de efeito devido ao SES em todos os desfechos para pelo menos um dos poluentes estudados. Indivíduos com piores indicadores socioeconômicos apresentaram um risco adicional de mortalidade por causas não acidentais, cardiovasculares e respiratórias em relação aos com melhores indicadores devido à exposição à poluição do ar. Reportou-se também que mães com menor nível educacional e as que moram em distritos mais desfavorecidos apresentaram maior probabilidade de sofrer prematuridade ou BPNT em relação às mães com alto nível de alfabetização e que moram em distritos com alto SES devido à exposição à poluição do ar, após controlar a temperatura, idade materna, sexo do recém-nascido, tipo de parto e número de consultas de pré-natal. Os resultados mostram que o SES individual e o contextual estão associados a uma maior chance de prematuridade e ainda maior para BPNT. Além disso, foi observado que, para o BPNT, ambos os indicadores socioeconômicos interagiram com a exposição a poluentes do ar em todos os períodos de gestação.

Para os desfechos de mortalidade, os resultados encontrados concordam com a literatura internacional. Pinault et al. (2017a), em uma coorte canadense, relataram uma modificação do efeito da educação na associação entre $\mathrm{MP}_{2.5} \mathrm{e}$ a mortalidade não acidental, em que indivíduos com escolaridade menor do que o ensino médio tinham maior risco de morrer do que o grupo que tinha mais anos de estudo (taxa de risco $(H R)=1,060 ;$ IC95\% 1,042, 1,079). Um estudo de série temporal de Kloog et al. (2013), nos EUA, relatou maior risco de morte por causas cardiorrespiratórias em indivíduos com 0 a 12 anos de escolaridade do que aqueles com mais de 12 anos para um aumento de $10 \mu \mathrm{g} / \mathrm{m}^{3}$ na exposição a $\mathrm{MP}_{2,5}(\mathrm{OR}=1,90 \mathrm{IC} 95 \%$ 1,60, 2,10 na menor escolaridade versus OR 1,40 IC95\% 1,20, 1,60 na maior). 
Blanco-Becerra et al. (2014) relataram, em um estudo ecológico na Colômbia, que distritos da categoria de SES intermediária apresentaram uma mudança de risco relativo percentual $(\mathrm{R} R \%)$ mais alta do que os distritos menos desfavorecidos para mortalidade respiratória associado a um aumento de 10 $\mu \mathrm{g} / \mathrm{m}^{3}$ na exposição ao $\mathrm{MP}_{10}$ ( $\mathrm{RR} \%$ variou de $1,31 \%$ a $1,77 \%$ em vários modelos). Richardson et al. (2011), em um estudo transversal na Nova Zelândia, também relataram maior risco de mortalidade nas áreas mais carentes (razão da taxa de incidência $(\mathrm{TIR})=3,46$ IC95\% 2,98; 4,02 áreas menos carentes como referência) devido a um aumento de $10 \mu \mathrm{g} / \mathrm{m}^{3}$ na exposição ao $\mathrm{MP}_{10}$ para mortalidade por causas respiratórias. De acordo com Barceló et al. (2009), em seu estudo transversal na Espanha, homens residentes em localidades de SES baixos e intermediários apresentaram maior chance de mortalidade por causas respiratórias devido à exposição ao $\mathrm{MP}_{10}$ (RR variando de 1,21 a 1,52) em relação a aqueles que viviam em localidades menos desfavorecidas. A modificação do efeito devido à exposição de curto prazo ao $\mathrm{NO}_{2}$ na mortalidade por todas as causas foi documentada por Deguen et al. (2015) em seu estudo de case-crossover na França, onde os indivíduos que vivem nos blocos censitários menos favorecidos tiveram riscos estatisticamente mais altos de morrer em comparação com os médios e menos carentes (Excesso de risco\% $(\mathrm{ER} \%)=3,1495 \% \mathrm{Cl} 1,41,4,90)$.

Outros estudos relataram modificação do efeito na maior escolaridade, como Zhang et al. (2014), em uma coorte chinesa, que encontraram maior risco de mortalidade cardiovascular associada a um aumento de $10 \mu \mathrm{g} / \mathrm{m}^{3}$ na exposição de $M P_{10}$ no grupo de ensino superior $(R R=1,52$ IC95\% 1,41, 1,65 em relação à $R R=1,19$ IC95\% 1,15 1,22 na categoria baixa educação).

Por outro lado, muitos estudos objetivaram encontrar modificação de efeito do SES individual na associação entre poluentes atmosféricos e mortalidade (O'Neill et al., 2008; Ren et al., 2010; Ou et al., 2008; Jerret et al., 2009; Faustini et al., 2016), mas não relataram evidências da hipótese levantada. A comparação entre estimativas de impactos na saúde de outros estudos pode não ser completamente viável devido às diferenças na mistura de poluentes do ar, sistema de saúde, clima e definição de SES. 
Já em relação aos desfechos adversos da gravidez, outros estudos encontraram resultados semelhantes aos do presente estudo, apresentando modificação do efeito do SESV na associação poluição do ar-eventos negativos da gravidez. Yi et al. (2010), na Coréia do Sul, relataram que mães que vivem em distritos de baixa renda tiveram um aumento adicional de $3,12 \%$ na probabilidade de prematuridade (IC95\%, 0,17 - 6,15\%) para um aumento de 10 $\mu \mathrm{g} / \mathrm{m}^{3}$ na exposição ao $\mathrm{MP}_{10}$ durante $\circ 2^{\circ}$ trimestre de gestação. Padula et al. (2014), nos EUA, também relataram um efeito semelhante na prematuridade associado a um aumento de $42,77 \mu \mathrm{g} / \mathrm{m}^{3}$ na exposição ao $\mathrm{MP}_{10}$ no segundo trimestre de gestação $(O R=1,13, I C 95 \%=1,06-1,22)$ e também durante todo o período de gestação $(O R=1,13, I C 95 \%=1,05-1,21)$ em mães que moram em bairros com piores condições socioeconômicas. Por outro lado, também no Canadá, Stieb et al. (2016) relataram que a associação entre $\mathrm{MP}_{2.5}$ e BPNT não era sensível ao ajuste do SES de vizinhança.

Gray et al. (2014), nos EUA, relataram que o efeito do $M_{2.5}$ na prematuridade e no BPN foi modificado pelo SES individual (educação materna) e no nível da área (renda mediana da área de residência baseada em censo). Eles relataram que mães com menos de 12 anos de estudo apresentaram maior risco de BPN e prematuridade (OR = 1,14-1,25 para BPN e 1,05-1,12 para prematuridade) e mães que residiam em domicílios de áreas com baixa renda mediana também tiveram maior chance de $\operatorname{BPN}(\mathrm{OR}=1,00-1,08)$, mas não para prematuridade $(O R=0,98-1,04)$.

A plausibilidade para indivíduos com pior SES serem mais suscetíveis a poluentes do ar foi sugerida anteriormente por O'Neill et al. (2003) e foi proposto que o baixo nível socioeconômico pode indicar piores condições de moradia, maior nível de poluição dentro da residência, menor acesso a alimentos saudáveis e água limpa e estilo de vida não saudável como o tabagismo e alcoolismo, levando a uma predisposição para obesidade, diabetes e outras comorbidades, aumentando sua vulnerabilidade à poluição do ar e, consequentemente, o risco de resultados adversos na gravidez. A educação materna mais alta pode indicar uma melhor compreensão dos efeitos adversos de vários agentes ambientais, estilo de vida mais saudável e maior acesso a 
serviços de saúde, o que poderia reduzir o impacto da poluição do ar nos resultados da gravidez (Šrám et al., 2005).

As diferenças entre os resultados relatados neste estudo e a literatura podem ser devido a diferenças na avaliação da exposição à poluição do ar, tamanho da amostra ou definição do SESV. Foram encontradas algumas associações opostas à hipótese inicial para exposição ao $\mathrm{NO}_{2}$ no $1^{\circ}$ e $2^{\circ}$ trimestres na prematuridade. Padula et al. (2014) levantaram a hipótese de que essas associações podem ocorrer devido a um viés de seleção, no qual apenas os nascidos vivos são incluídos. Portanto, se a exposição ao $\mathrm{NO}_{2}$ no início das gestações tiver uma associação mais forte com a mortalidade fetal do que com a prematuridade, acabaria-se examinando apenas aqueles que sobreviveram ao período em que a mortalidade seria maior. Estudos futuros devem investigar ainda mais a associação entre poluição do ar e mortalidade fetal.

Este estudo teve vários pontos fortes, como o grande número amostral para ambos os desfechos, dados municipais de vários anos provenientes das certidões de óbito e nascimento, informações residenciais e um modelo estatístico robusto, entretanto também teve algumas limitações. Não foi possível medir a exposição exata à poluição do ar em cada indivíduo falecido nem em cada gravidez. O endereço residencial materno no momento do nascimento pode não ser o mesmo que a mãe tinha durante a gestação e também não foi possível coletar informações sobre a mobilidade materna durante a gravidez, o que pode ter levado a um viés de exposição não diferencial. Isso implicaria que a magnitude dos resultados pode ter sido subestimada. Além disso, não foi possível controlar o hábito materno de fumar, o consumo de álcool, o estado nutricional e a ingestão de drogas, embora o consumo de álcool e drogas possa ter sido indiretamente ajustado pelo nível educacional (O'Neill et al., 2003; Barbieri et al., 2000). Além disso, não foi possível obter informações sobre morbidades maternas, como diabetes ou hipertensão, que podem levar a eventos adversos na gestação (Yorifuji et al., 2013; Padula et al., 2014). As certidões de nascimento não indicavam se os partos prematuros foram espontâneos ou indicados. Finalmente, não foi possível controlar completamente a heterogeneidade espacial que os distritos possuem. 


\section{CONCLUSÃO}

Em conclusão, este estudo encontrou evidências de que o SES em nível individual e de vizinhança modifica o efeito da exposição a curto prazo ao $\mathrm{O}_{3}$, $\mathrm{MP}_{10}$ e $\mathrm{NO}_{2}$ na mortalidade diária por causas não acidentais, cardiovasculares e respiratórias. Concluiu-se também que maior exposição ao $\mathrm{O}_{3}$ durante toda a gravidez aumentou as chances de prematuridade em $14 \%$ e a exposição ao MP 10 aumentou as chances de prematuridade e BPNT em 8\%. Além disso, considerando todo o período de gravidez, as mães com baixa escolaridade tiveram um risco adicional de $10 \%$ de BPNT devido à exposição ao $\mathrm{MP}_{10}$ e as que moravam em distritos com piores condições socioeconômicas tiveram um risco adicional de $14 \%$ de prematuridade devido à exposição ao $\mathrm{O}_{3}$. Esses resultados fornecem evidências adicionais de que o SES individual e em nível de vizinhança modificam o efeito da poluição do ar na prematuridade e BPNT. 


\section{ANEXOS}

8.1 Anexo A Artigo modificação de efeito do SES na mortalidade

TITLE: Case-crossover analysis of air pollution and mortality: the role of socioeconomical status

Felipe Parra do Nascimento ${ }^{1, *}$, Nelson Gouveia ${ }^{1}$

1 School of Medicine, University of São Paulo, São Paulo, Brazil.

* Corresponding Author - Felipe Parra do Nascimento,

Departamento de Medicina Preventiva, Faculdade de Medicina, Universidade de São Paulo, Av. Dr. Arnaldo, n455, São Paulo/SP, Brasil.

E-mail: felipe.parra.nascimento@usp.br

Versão preliminar do artigo 
BACKGROUND: There is a vast literature covering the association between air pollution exposure and non-accidental and cardiorespiratory mortality. However, it is still not clear if this effect is modified by socioeconomic status (SES).

OBJECTIVES: We investigated if individual and area-level socioeconomic status modified the relationship between short-term exposure to ozone $\left(\mathrm{O}_{3}\right)$, nitrogen dioxide $\left(\mathrm{NO}_{2}\right)$ and particulate matter with aerodynamic diameter $<10 \mu \mathrm{m}\left(\mathrm{PM}_{10}\right)$ on cardiovascular, respiratory and non-accidental mortality.

METHODS: Analyses were based on individual information of 280685 individuals that passed away from 2011 to 2015 period in São Paulo municipality. Exposure to $\mathrm{PM}_{10}, \mathrm{NO}_{2}$ and $\mathrm{O}_{3}$ were accessed from monitoring stations and linked to each case based on date of death. We conducted a time-stratified case-crossover approach. Conditional Logistic Models were used to estimate the main effects of air pollutants and an interaction term was added in the models to access the effect modification of SES on all outcomes.

RESULTS: In fully adjusted models, a $10 \mu \mathrm{g} / \mathrm{m}^{3}$ increase in $\mathrm{O}_{3}$ and $\mathrm{NO}_{2}$ was statistically associated to all outcomes but not $\mathrm{PM}_{10}$. Individuals living on low SES areas had an additional risk of dying for non-accidental and cardiovascular causes $(0.63 \% 95 \% \mathrm{Cl} 0.27 \%, 0.99 \% ; 0.68 \% 95 \% \mathrm{Cl} 0.06 \%, 1.29 \%)$ and living on mid SES areas for respiratory $(1.70 \% 95 \% \mathrm{Cl} 0.75 \%, 2.65 \%)$ due to $\mathrm{NO}_{2}$ exposure compared to individuals living on high SES areas. Individuals with 9 to 11 years of study had and additional risk of dying of respiratory causes due to exposure to $\mathrm{O}_{3}(0.59 \% 95 \% \mathrm{Cl} 0.05 \%, 1.13 \%)$ in comparison to individuals with 12 or more years of study. Although we found no main effect for PM10 exposure we found a cross-over interaction for individuals with 0 to 8 years of study for nonaccidental and cardiovascular deaths $(1.29 \% 95 \% \mathrm{Cl} 0.34 \%, 2.25 \%$ and $2.03 \%$ $95 \% \mathrm{Cl} 0.29 \%, 3.76 \%$ respectively) and on the mid SES areas for respiratory $(2.80 \% 95 \% \mathrm{Cl} 0.98 \%, 4.64 \%)$.

CONCLUSION: Socioeconomic status modifies the effect of air pollution on nonaccidental, cardiovascular and respiratory mortality. Results indicates that 
individuals living on lower SES areas may be more susceptible to air pollution effects.

\section{Introduction}

Air pollution has been identified as a serious public health problem since the beginning of $20^{\text {th }}$ century. The main sources of emission include industrial process and burning of fossil fuels (Braga et al., 2007). The effects of air pollution exposure appear even when their levels are below AQG standards (Bakonyi et al., 2007). Age groups more affected are children (Ghosh et al., 2013; Braga et al., 1999; Carbajal-Arroyo et al., 2011) and elderly (Cakmak et al., 2011; Martins et al., 2002; Yang et al., 2018). Various studies documented associations between air pollutants such as nitrogen dioxide $\left(\mathrm{NO}_{2}\right)$, particulate matter with aerodynamic diameter $<10 \mu \mathrm{m}\left(\mathrm{PM}_{10}\right)$, ozone $\left(\mathrm{O}_{3}\right)$, sulphur dioxide $\left(\mathrm{SO}_{2}\right)$ and carbon monoxide (CO) and adverse health outcomes (Deguen et al., 2015).

Many recent studies have been interested in evaluating how air pollution exposure and socioeconomical status (SES) correlate with mortality and if SES modifies the effects of air pollution in the population. The main hypotheses are that either poorer people are more exposed to air pollution directly in their jobs or living near industrial parks (environmental injustice) or that individuals with lower SES become more vulnerable to the air pollution harmful effects because of limited access to healthy food and water, limited access to health care services and worse lifestyle habits such as smoking, drinking, etc. (Wong et al., 2008; Qiu et al., 2015; Deguen et al., 2010).

Some studies already reported that both individual and area-level SES modify the effects of air pollution in the health of the population. Bravo et al. (2016), in a case-crossover study conducted in São Paulo, Brazil, reported that non-accidental mortality associated with exposure to $\mathrm{NO}_{2}, \mathrm{SO}_{2}$ and $\mathrm{CO}$ was higher in the low education group, and a similar pattern when examining cardiovascular mortality and $\mathrm{PM}_{10}$ exposure. Brunt et al. (2016), on Wales, UK, reported increased chance of dying due to all-cause and respiratory diseases by $\mathrm{PM}_{10}, \mathrm{PM}_{2.5}$ and $\mathrm{NO}_{2}$ exposure on low SES areas. A study conducted in China reported that individuals with low educational attainment were more vulnerable to $\mathrm{PM}_{10}$ exposure regarding all-cause mortality (Chen et al., 2012). Deguen et al., 
(2015), on Paris, France, reported higher risk of all-cause mortality due to exposure to $\mathrm{NO}_{2}$ among individuals living on most deprived census blocks. Wang and collaborators (2017), in a cohort conducted USA, reported that the association between long-term exposure to $\mathrm{PM}_{2.5}$ and all-cause mortality was modified by area-level SES, being the low SES group more susceptible to the adverse effects caused by the pollutant. In other studies, it has been reported association between air pollution and adverse health effects but no SES modification (Ren et al., 2010; Chi et al., 2016; Jerrett et al., 2009; Dong et al., 2012; O'Neill et al., 2008) while in others SES modified the air pollution effects on the higher SES population (Zhang et al., 2014; Schifano et al., 2013).

The present study will investigate if individual and area-level SES modifies the association between air pollution and mortality in São Paulo, Brazil. Based on previous evidence, we have chosen $\mathrm{PM}_{10}, \mathrm{O}_{3}$ and $\mathrm{NO}_{2}$ as pollutants to have the risk estimated and populational mortality by all non-accidental causes of death, respiratory deaths and cardiovascular deaths as outcomes.

\section{Materials and Methods}

Study area and design: This study was carried out in the municipality of São Paulo, Brazil's largest city with a population of over 12 million people in 2017 and a demographic density of 7960 inhabitants $/ \mathrm{Km}^{2}$ (IBGE, 2017). To study the effects of air pollution on this population we conducted a time-stratified casecrossover study.

Mortality Data: Daily deaths data were collected by Programa de Aprimoramento das Informações de Mortalidade (PRO-AIM), São Paulo's municipal Health Secretary program, from January $1^{\text {st }} 2011$ to December $31^{\text {st }} 2015$. We obtained information from death certificates about individual age, gender, date of death, schooling, district of residence and main cause of death according to ICD-10. The outcomes analyzed were deaths by all non-accidental causes (A00 - T98, Z00 Z99), due to respiratory (J00 - J98) and cardiovascular diseases (100 - 199). We excluded individuals less than 30 years old for all analysis. 
Air pollution data: Data for air pollution were extracted directly from Companhia Ambiental do Estado de São Paulo (CETESB, http://qualar.cetesb.sp.gov.br/qualar/home.do) Monitoring Network System from January $1^{\text {st }} 2010$ to December $31^{\text {st }} 2015$. We included the year of 2010 because deaths that happened in early 2011 had air pollution exposure in 2010. We obtained the daily mean value for $\mathrm{PM}_{10}$ and $\mathrm{NO}_{2}$ and the highest 8-hour moving average for $\mathrm{O}_{3}$. Daily air pollutant measures were considered valid when at least 16 of the 24-hourly measurements had no errors. Because correlation between monitoring stations values for each pollutant were very high (see Supplementary Material, tables 1 to 3), when data were available for more than one monitoring station, the values were averaged.

Meteorological Data: Daily average temperature and relative humidity were obtained from Instituto de Astronomia, Geofísica, e Ciências Atmosféricas da Universidade de São Paulo (http://www.estacao.iag.usp.br/contatos.php) for January $1^{\text {st }} 2010$ to December $31^{\text {st }} 2015$ period.

Socioeconomic status: We used education as individual SES indicator which was divided into 3 categories: 0 to 8, 9 to 11 and 12 or more years of study. For arealevel SES indicator, we created a socioeconomic index using information from the Census of 2010 data (http://infocidade.prefeitura.sp.gov.br) for each of the 96 districts of São Paulo. The index was constructed by applying principal components analysis with varimax rotation to the following variables: mean income; literacy rate; one-person housing; education (mean years of education for head of household); housing conditions (proportion of residences not connected to sewage network). All variables were first standardized to remove the influence of different units of measurement. The first component explained $65.5 \%$ of the variance and was used to build the socioeconomic index. Each district received a score level based in the socioeconomic index and was ranked and then divided into three tertiles. The 32 highest scores were allocated in the high SES category, the next 32 into the medium SES and the lowest 32 in the low SES. Results were consistent with previous reports (Takiya et al., 2002). This method has been previously used on other studies with some variations (Brunt et 
al., 2016; Blanco-Becerra et al., 2014; Carbajal-Arroyo et al., 2011; Deguen et al., 2015).

Statistical analysis: We used a case-crossover design where each case acts as its own control. We used time-stratified referent selection which allowed us to control potential confounding variables such as age, day of the week, seasonality and temperature. The controls days were selected within 1 month of death, leaving 7 days to match the same day of the week before the event $(7,14,21$ and 28 days prior to the event) and to eliminate the 6-day autocorrelation between all observations used in the analysis as well as seasonality (Levy et al., 2001) which gave us 4 control periods per case.

First, we estimated the risk of mortality for non-accidental, cardiovascular and respiratory deaths due to exposure to $\mathrm{PM}_{10}, \mathrm{NO}_{2}, \mathrm{O}_{3}$ through a multiple conditional logistic regression adjusting the effect of education and area-SES by temperature and relative humidity. Pollutants entered the models as continuous variables on single-pollutant models. Several lags of effects for the pollutants were tested (current day of death (lag 0), the day prior (lag1) and so on) up to 5 days prior to the events. We also considered various cumulative average lag periods (lag $0-1$ is the average of the pollutant of the day of death and prior to it, lag $0-2$ is the average of the 3 days, etc.) while controlling for daily mean temperature and humidity.

Finally, we tested whether those effects were modified by individual and area-level SES by introducing a multiplicative interaction term in the model, using the same lag structure selected in the previous step, with high SES (both individual and area-level) as the reference category, adjusting for age, gender, temperature and relative humidity.. Results are reported as odds ratios (OR) for a change of $10 \mu \mathrm{g} / \mathrm{m}^{3}$ of the pollutant in the case period in relation to the control period, followed by the $95 \%$ confidence interval (IC95\%) for each estimative. All statistical analyses were performed on Stata version 13.0.

\section{Results}


From January $1^{\text {st }} 2011$ to December $31^{\text {st }} 2015$ we had a total of 311,574 deaths in São Paulo municipality for individual over 30 years of age. We excluded individuals that had no information about educational level in the death certificates (30889 or $9.9 \%$ ) from all analyses. Since all other studied variables had no missing data, our final study population was 280685 individuals. Subjects mostly had between 75 and 109 years (47.1\%), between 0 and 8 years of study $(70.9 \%)$ and lived on low area-SES districts (38.4\%). Similar proportions were observed for cardiovascular and respiratory outcomes (table 1). Mean age for all non-accidental causes was 71.1 years $( \pm 15.3)$, similar to cardiovascular and respiratory outcomes. Women were on average 5.7 years older than men and less educated, (12.6\% had 12 or more years of study versus $7.9 \%)$. There were more females than males that lived on high SES districts (29.9 versus $25.7 \%$ ). Mean age among low education group was 72.8 years while on high education it was 68.7 years. Mean age of individuals living on low-SES districts was 66.7 years while on high SES districts was 76.4 years (data not shown).

Mean daily levels of $\mathrm{PM}_{10}$ were $38.7 \mu \mathrm{g} / \mathrm{m}^{3}\left( \pm 16.0 \mu \mathrm{g} / \mathrm{m}^{3}\right)$ during the study period, $\mathrm{NO}_{2}$ were $78.9 \mu \mathrm{g} / \mathrm{m}^{3}\left( \pm 33.5 \mu \mathrm{g} / \mathrm{m}^{3}\right)$ and highest daily 8-hour moving average of $\mathrm{O}_{3}$ was $69.4 \mu \mathrm{g} / \mathrm{m}^{3}\left( \pm 32.0 \mu \mathrm{g} / \mathrm{m}^{3}\right)$, mean daily temperature was around $20^{\circ} \mathrm{C}$ and relative humidity around $80 \%$ (table 2 ). Correlation between pollutants was high for $\mathrm{PM}_{10}$ and $\mathrm{NO}_{2}$ (Pearson $\mathrm{r}=0.750$ ) but not so high for the other pollutants. $\mathrm{O}_{3}$ was averagely correlated to temperature and inversely correlated to relative humidity (Supplementary Material, Table S4).

We estimated the main effects of the pollutants on non-accidental, cardiovascular and respiratory mortality of adults in São Paulo municipality, adjusting for age, gender, temperature and relative humidity. We tested multiple lag days (0 to 5) and average values (lags 0-1, lag 0-2, until 0-5) and selected the models based on lowest log-likelihood values. We found positive associations between a $10 \mu \mathrm{g} / \mathrm{m}^{3}$ increase on $\mathrm{O}_{3}$ and $\mathrm{NO}_{2}$ levels and percent chance of mortality for all outcomes but not for $\mathrm{PM}_{10}$ exposure (table 3 ). We found a statistically significant effect modification of education on $\mathrm{O}_{3}$ in the category of 9 to 11 years for non-accidental causes of death and a cross-over interaction in the 0 to 8 years category for $\mathrm{PM}_{10}$ exposure on non-accidental and cardiovascular 
outcomes. We also found a statistically significant effect modification of area-SES on both low and middle categories by $\mathrm{NO}_{2}$ exposure on non-accidental deaths, on low districts for $\mathrm{NO}_{2}$ exposure on cardiovascular outcomes and middle SES districts for $\mathrm{PM}_{10}$ and $\mathrm{NO}_{2}$ exposure for respiratory outcomes (tables 4 to 6).

\section{Discussion}

Our study found evidence that both individual and area-level SES modifies the effect of short-term exposure to $\mathrm{O}_{3}, \mathrm{PM}_{10}$ and $\mathrm{NO}_{2}$ on daily mortality from nonaccidental, cardiovascular and respiratory causes.

Our findings agree with previous results reported on international studies. Pinault et al. (2017), in a Canadian cohort, reported an effect modification of education on $\mathrm{PM}_{2.5}$ exposure to non-accidental mortality where individuals with schooling lower than high school had higher risk of dying than the group that had more years of study (hazard ratio $(\mathrm{HR})=1.060 ; 95 \% \mathrm{Cl} 1.042,1.079$ ). A timeseries study by Kloog et al. (2013), on USA, reported higher risk os death for cardiorespiratory causes on individuals with 0 to 12 years of schooling than those with more than 12 years for an increase of $10 \mu \mathrm{g} / \mathrm{m}^{3}$ on $\mathrm{PM}_{2.5}$ exposure (OR = $1.9095 \% \mathrm{Cl} 1.60,2.10$ on lower education versus OR $1.4095 \% \mathrm{Cl} 1.20,1.60$ ).

Blanco-Becerra et al. (2014) reported, on an ecological study in Colombia, that locations of middle area-level SES presented a higher relative risk change $(\mathrm{RR} \%)$ than high group for respiratory mortality associated to an increase of 10 $\mathrm{\mu g} / \mathrm{m}^{3}$ in $\mathrm{PM}_{10}$ exposure (RR\% varied from $1.31 \%$ to $1.77 \%$ on various models). Richardson et al. (2011), in a cross-sectional study in New Zealand, also reported higher risk of mortality on the most deprived areas (incidence rate ratio (IRR) $=$ $3.4695 \% \mathrm{Cl} 2.98,4.02$ less deprived areas were the reference) due to an increase of $10 \mu \mathrm{g} / \mathrm{m}^{3}$ in $\mathrm{PM}_{10}$ exposure for respiratory outcomes. According to Barceló et al. (2009), on their cross-sectional study in Spain, men residing on medium and low SES locations had higher chance of mortality from respiratory causes due to $\mathrm{PM}_{10}$ exposure (RR varying 1.21 to 1.52 in a gradient trend). Effect modification due to short-term exposure to $\mathrm{NO}_{2}$ on all-cause mortality has been 
documented by Deguen et al. (2015) on their case-crossover study in France, where individuals living on most deprived census blocks had statistically higher risks of dying compared to mid and less deprived (Excess risk \% $(E R \%)=3.14$ $95 \% \mathrm{Cl} 1.41,4.90)$.

Other studies reported inverse effect modification such as Zhang et al. (2014), in a Chinese Cohort, that found higher risk ratio for cardiovascular mortality associated to an increase of $10 \mu \mathrm{g} / \mathrm{m}^{3}$ in the high education group (RR $=1.5295 \% \mathrm{Cl} 1.41,1.65$ in relation to $\mathrm{RR}=1.1995 \% \mathrm{Cl} 1.15,1.22$ in the low education category). It has also been reported effect modifications different from ours such as Brunt et al. (2016), in Wales, that found area-SES modification on most deprived areas having higher risk of non-accidental mortality due to $\mathrm{PM}_{10}$ exposure and Cakmak et al. (2011) that reported individual SES effect modification on lower education individuals having higher risk of mortality by nonaccidental causes due to exposure by $\mathrm{NO}_{2}$. On the other hand, many studies aimed to find individual SES effect modification of air pollutants on mortality (O'Neill et al., 2008; Ren et al., 2010; Ou et al., 2008; Jerret et al., 2009; Faustini et al., 2016) but reported no evidence of such. Comparison between estimates of health impacts from other studies may not be completely viable due to differences on air pollution mixture, health care system, climate and definition of SES. -> (essa ultima frase seria uma limitação?)

Plausibility for Individuals with lower SES be more susceptible to air pollutants has been previously suggested by O'Neill et al. (2003) and it was proposed that low SES may indicate worse housing conditions, which could also indicate higher indoor pollution, less access to healthy food and clean water, and unhealthy lifestyle such as smoking, leading to a predisposition to obesity, diabetes and other comorbidities.

Our study had several strengths such as the use of both individual data collected from death certificates and contextual information based on an index built using principal components that was able to divide socioeconomically the whole municipality population, although not completely homogeneous within each category, made it possible to estimate the reality of São Paulo city. Our 
study had some limitations. It was not possible to measure each individual exposure to pollution, since we could not access occupational exposure nor smoking habits, though it may be indirectly adjusted by education (O'Neill et al. 2003). Death certificates were filled with residence at time of death which may not reflect properly where the individual spent most of his time living, leading to a non-differential exposure misclassification, underestimating the magnitude of our results.

In conclusion, we report further evidence for the association between $\mathrm{O}_{3}$ and $\mathrm{NO}_{2}$ short-term exposure and non-accidental, cardiovascular and respiratory mortality. We were also able to detect effect modification of both individual and area-level SES on air pollution-mortality relationship.

\section{Conflicts of interest}

The authors have no conflicts of interest to address.

\section{Funding...}

This study was financed in part by the Coordenação de Aperfeiçoamento de Pessoal de Nível Superior - Brasil (CAPES) - Finance Code 001.

\section{References}

Bakonyi SMC, Danni-Oliveira IM, Martins LC, Braga ALF. Air pollution and respiratory diseases among children in Brazil. Rev Saude Publica. 2004; 38(5): $1-5$.

Barceló MA, Saez M, Saurina C. Spatial variability in mortality inequalities, socioeconomic deprivation, and air pollution in small areas of the Barcelona Metropolitan Region, Spain. Sci Total Environ. 2009;407:5501-23.

Blanco-Becerra LC, Miranda-Soberanis V, Barrazza-Villarreal A, Junger W, Hurtado-Díaz M, Romieu I. Effect of socioeconomic status on the association 
between air pollution and mortality in Bogota, Colombia. Salud Publica Mex. 2014;56(4):371-8.

Braga ALF, Conceição GMS, Pereira LAA, Kishi HS, Pereira JCR, Andrade MF, et al. Air pollution and pediatric respiratory hospital admissions in São Paulo, Brazil. J Environ Med. 1999;1:95-102.

Braga ALF, Pereira LAA, Procópio M, André PA, Saldiva PHN. Associação entre poluição atmosférica e doenças respiratórias e cardiovasculares na cidade de Itabira, Minas Gerais, Brasil. Cad Saude Publica. 2007; 23(4): 5570-8.

Bravo MA, Son J, Freitas CU, Gouveia N, Bell ML. Air pollution and mortality in São Paulo, Brazil: effects of multiple pollutants and analysis of susceptible populations. J Expo Sci Environ Epidemiol. 2016;26:150-61.

Brunt H, Barnes J, Jones SJ, Longhurst JWS, Scally G, Hayes E. Air pollution, deprivation and health: understanding relationships to add value to local air quality management policy and practice in Wales, UK. J Public Health (Oxf). 2016;39(3):485-97.

Cakmak S, Dales RE, Rubio MA, Vidal CB. The risk of dying on days of higher air pollution among the socially disadvantaged elderly. Environ Res. 2011;111:388-93.

Carbajal-Arroyo L, Miranda-Soberanis V, Medina Ramón M, Rojas-Bracho L, Tzintzun G, Solís-Gutiérrez $\mathrm{P}$, et al. Effect of $\mathrm{PM}_{10}$ and $\mathrm{O}_{3}$ on infant mortality among residents in the Mexico City Metropolitan Area: a case-crossover analysis, 1997-2005. J Epidemiol Community Health. 2011;65(8):715-21.

Chen R, Kan H, Chen B, Huang W, Bai Z, Song G, et al. Association of particulate air pollution with daily mortality: the China air pollution and health effects study. Am J Epidemiol. 2012;175(11):1173-81.

Chi GC, Hajat A, Bird CE, Cullen MR, Griffin BA, Miller KA, et al. Individual and neighborhood socioeconomic status and the association between air pollution and cardiovascular disease. Environ Health Perspect. 2016;124(12):1840-47. 
Deguen S, Petit C, Delbarre A, Kihal W, Padilla C, Benmarhnia T, et al. Neighbourhood characteristics and long-term air pollution levels modify the association between the short-term nitrogen dioxide concentrations and all-cause mortality in Paris. PloS One. 2015;10(7):[14 p.]

Deguen S, Zmirou-Navier D. Social inequalities resulting from health risks related to ambient air quality - an european review. Eur J Public Health. 2010;20(1):2735.

Dong GH, Zhang P, Sun B, Zhang L, Chen X, Ma N, et al. Long-term exposure to ambient air pollution and respiratory disease mortality in Shenyang, China: a 12-year population-based retrospective cohort study. Respiration. 2012;84:3608.

Faustini A, Stafoggia M, Renzi M, Cesaroni G, Alessandrini E, Davoli M, et al. Does chronic exposure to high levels of nitrogen dioxide exacerbate the shortterm effects of airbone particles? Occup Environ Med. 2016;73:772-8.

Ghosh JKC, Heck JE, Cockburn M, Su J, Jerrett M, Ritz B. Prenatal exposure to traffic-related air pollution and risk of early childhood cancers. Am J Epidemiol. 2013;178(8):1233-9.

Instituto Brasileiro de Geografia e Estatística [Internet]. Rio de Janeiro: IBGE Instituto Brasileiro de Geografia e Estatística; c2017 [cited 2018 May 10]. São Paulo [municipality]: panorama. Available from: https://cidades.ibge.gov.br/brasil/sp/sao-paulo/panorama

Jerrett M, Burnett RT, Pope CA 3rd, Ito K, Thurston G, Krewski D, et al. Longterm ozone exposure and mortality. N Engl J Med. 2009;360(11):1085-95.

Kloog I, Ridgway B, Koutrakis P, Coull BA, Schwartz JD. Long- and short-term exposure to $\mathrm{PM}_{2.5}$ and mortality: using novel exposure models. Epidemiology. 2013;24(4):555-61. 
Levy D, Lumley T, Sheppard L, Kaufman J, Checkoway H. Referent selection in case-crossover analysis of acute health effects of air pollution. Epidemiology. 2001;12(2):186-92

Martins LC, Latorre MRDO, Cardoso MRA, Gonçalves FLT, Saldiva PHN, Braga ALF. Poluição atmosférica e atendimentos por pneumonia e gripe em São Paulo, Brasil. Rev Saude Publica. 2002;36:88-94.

O'Neill MS, Bell ML, Ranjit N, Cifuentes LA, Loomis D, Gouveia N, et al. Air pollution and mortality in Latin America: the role of education. Epidemiology. 2008;19(6):810-9.

O’Neill MS, Jerrett M, Kawachi I, Levy JI, Cohen AJ, Gouveia N, et al. Health, wealth, and air pollution: advancing theory and methods. Environ Health Perspect. 2003;111(16):1861-70.

Ou CQ, Hedley AJ, Chung RY, Thach TQ, Chau YK, Chan KP, et al. Socioeconomic disparities in air pollution-associated mortality. Environ Res. 2008;107:237-44.

Pinault LL, Weichenthal S, Crouse DL, Brauer M, Erickson A, Van Donkelaar A, et al. Associations between fine particulate matter and mortality in the 2001 Canadian Census Health and Environment Cohort. Environ Res. 2017;159:40615.

Qiu H, Tian L, Ho KF, Pun VC, Wang X, Yu ITS. Air pollution and mortality: effect modification by personal characteristics and specific cause of death in a caseonly study. Environ Pollut. 2015;199:192-7.

Ren C, Melly S, Schwartz J. Modifiers of short-term effects of ozone on mortality in eastern Massachusetts: a case-crossover analysis at individual level. Environ Health. 2010;9:3.

Richardson EA, Pearce J, Kingham S. Is particulate air pollution associated with health and health inequalities in New Zealand? Health Place. 2011;17:1137-43. 
Schifano P, Lallo A, Asta F, De Sario M, Davoli M, Michelozzi P. Effect of ambient temperature and air pollutants on the risk of preterm birth, Rome 2001-2010. Environ Int. 2013;61:77-87.

Takiya H. Atlas Ambiental do Município de São Paulo: fase I: diagnóstico e bases para a definição de políticas públicas para as áreas verdes no município de São Paulo. São Paulo: Prefeitura do Município de São Paulo. Secretaria Municipal do Meio Ambiente, Secretaria Municipal de Planejamento Urbano; 2002 Jul. Relatório final. Processo n: 1999/10955-9. Projeto Biota - FAPESP.

Wang Y, Shi L, Lee M, Liu P, Di Q, Zanobetti A, et al. Long-term exposure to $\mathrm{PM}_{2.5}$ and mortality among older adults in the Southeastern US. Epidemiology. 2017;28(2):207-14.

Wong CM, Ou CQ, Chan KP, Chau YK, Thach TQ, Yang L, et al. The effects of air pollution on mortality in socially deprived urban areas in Hong Kong, China. Environ Health Perspect. 2008;116(9):1189-94.

Yang Y, Tang R, Qiu H, Lai PC, Wong P, Thach TQ, et al. Long term exposure to air pollution and mortality in an elderly cohort in Hong Kong. Environ Int. 2018;117:99-106.

Zhang LW, Chen X, Xue XD, Sun M, Han B, Li CP, et al. Long-term exposure to high particulate matter pollution and cardiovascular mortality: a 12-year cohort study in four cities in northern China. Environ Int. 2014;62:41-7. 
Table 1: Descriptive Statistics for the study population, São Paulo, 2011 - 2015:

\begin{tabular}{lccc} 
& non-accidental N (\%) & Cardiovascular N (\%) & Respiratory N (\%) \\
\hline Total & 280685 & 102032 & 40973 \\
Age Group & & & \\
$30-64$ & $91859(32.7)$ & $31038(30.4)$ & $8780(21.5)$ \\
$65-74$ & $56701(20.2)$ & $21388(21.0)$ & $7254(17.8)$ \\
$75-109$ & $132125(47.1)$ & $49606(48.6)$ & $24759(60.7)$ \\
& & & \\
Gender & & & $20716(50.8)$ \\
$\quad$ Male & $140594(50.1)$ & $51200(50.2)$ & $20077(49.2)$ \\
Female & $140091(49.9)$ & $50832(49.8)$ & \\
& & & $31032(76.1)$ \\
Education & & & $6408(15.7)$ \\
Low & $199086(70.9)$ & $75027(73.5)$ & $3353(8.2)$ \\
$\quad$ Medium & $52755(18.8)$ & $18503(18.1)$ & \\
High & $28844(10.3)$ & $8502(8.4)$ & $15137(37.1)$ \\
Area-SES & & & $13810(33.9)$ \\
Low & $107737(38.4)$ & $41994(41.2)$ & \\
$\quad$ Medium & $94851(33.8)$ & $34607(33.9)$ & \\
High & $78097(27.8)$ & $25431(24.9)$ & \\
\hline
\end{tabular}

Table 2: Air pollutants concentrations and meteorological measures, São Paulo, $2011-2015$

\begin{tabular}{lccccccc} 
& Mean & SD & Min & 25th & Median & 75th & Max \\
\hline $\mathrm{O}_{3}\left(\mu \mathrm{g} / \mathrm{m}^{3}\right)$ & 69.4 & 32.0 & 3.7 & 45.0 & 65.3 & 90.7 & 187.0 \\
$\mathrm{PM}_{10}\left(\mu \mathrm{g} / \mathrm{m}^{3}\right)$ & 38.7 & 16.0 & 9.5 & 27.0 & 35.7 & 46.7 & 110.7 \\
$\mathrm{NO}_{2}\left(\mu \mathrm{g} / \mathrm{m}^{3}\right)$ & 78.9 & 33.5 & 11.0 & 54.0 & 74.0 & 100.0 & 209.0 \\
Temperature $\left({ }^{\circ} \mathrm{C}\right)$ & 19.7 & 3.3 & 7.3 & 17.5 & 19.8 & 22.2 & 28.0 \\
Relative humidity (\%) & 79.8 & 8.9 & 41.2 & 74.9 & 80.8 & 86.0 & 96.9 \\
\hline
\end{tabular}


Table 3: Percent change (95\% confidence interval) of mortality associated with an increase of $10 \mu \mathrm{g} / \mathrm{m}^{3}$ in air pollutant concentration.

\begin{tabular}{lccc} 
& $\mathrm{O}_{3}(\operatorname{lag} 1)$ & $\mathrm{PM}_{10}(\operatorname{lag} 1)$ & $\mathrm{NO}_{2}(\operatorname{lag} 0)$ \\
\hline Non-accidental & $0.73(0.69 ; 0.78)$ & $0.77(-0.29 ; 1.73)$ & $1.37(1.33 ; 1.42)$ \\
Cardiovascular & $0.77(0.68 ; 0.85)$ & $0.97(-0.76 ; 2.70)$ & $1.34(1.25 ; 1.42)$ \\
Respiratory & $0.69(0.56 ; 0.83)$ & $1.28(-1.45 ; 4.02)$ & $1.32(1.19 ; 1.45)$ \\
\hline
\end{tabular}

Table 4: Percent change (95\% confidence intervals) of effect modification for non-external mortality associated with a $10 \mu \mathrm{g} / \mathrm{m}^{3}$ increase in air pollutant concentration

\begin{tabular}{llll} 
& $\mathrm{O}_{3}(\operatorname{lag} 1)$ & $\mathrm{PM}_{10}(\operatorname{lag} 1)$ & $\mathrm{NO}_{2}(\operatorname{lag} 0)$ \\
\hline Education & & & \\
$\quad$ 0-8 years & $0.36(-0.12 ; 0.84)$ & $1.29(0.34 ; 2.25)$ & $-0.49(-0.98 ;-0.06)$ \\
9-11 years & $0.59(0.05 ; 1.13)$ & $0.81(-0.26 ; 1.88)$ & $-0.28(-0.82 ; 0.27)$ \\
& & & \\
SES & & & \\
Low & $0.18(-0.18 ; 0.54)$ & $-0.15(-0.86 ; 0.56)$ & $0.63(0.27 ; 0.99)$ \\
$\quad$ Mid & $0.36(-0.04 ; 0.72)$ & $0.12(-0.60 ; 0.83)$ & $0.60(0.23 ; 0.97)$ \\
\hline
\end{tabular}

Table 5: Percent change (95\% confidence intervals) of effect modification for cardiovascular mortality associated with a $10 \mu \mathrm{g} / \mathrm{m}^{3}$ increase in air pollutant concentration

\begin{tabular}{llll} 
& $\mathrm{O}_{3}(\operatorname{lag} 1)$ & $\mathrm{PM}_{10}(\operatorname{lag} 1)$ & $\mathrm{NO}_{2}(\operatorname{lag} 0)$ \\
\hline Education & & & \\
$0-8$ & $0.50(-0.37 ; 1.37)$ & $2.03(0.29 ; 3.76)$ & $-0.09(-0.98 ; 0.78)$ \\
$9-11$ & $0.61(-0.36 ; 1.57)$ & $1.29(-0.62 ; 3.20)$ & $-0.43(-1.40 ; 0.54)$ \\
& & & \\
SES & & & \\
Low & $0.12(-0.48 ; 0.73)$ & $-0.62(-1.81 ; 0.56)$ & $0.68(0.06 ; 1.29)$ \\
$\quad$ Mid & $0.26(-0.35 ; 0.88)$ & $-0.48(-1.69 ; 0.73)$ & $0.30(-0.33 ; 0.92)$ \\
\hline
\end{tabular}

Table 6: Percent change (95\% confidence intervals) of effect modification for respiratory mortality associated with a $10 \mu \mathrm{g} / \mathrm{m}^{3}$ increase in air pollutant concentration

\begin{tabular}{llll} 
& $\mathrm{O}_{3}(\operatorname{lag} 1)$ & $\mathrm{PM}_{10}(\operatorname{lag} 1)$ & $\mathrm{NO}_{2}(\operatorname{lag} 0)$ \\
\hline Education & & & \\
$0-8$ & $0.55(-0.85 ; 1.95)$ & $-1.53(-4.20 ; 1.14)$ & $-0.44(-1.84 ; 0.96)$ \\
$9-11$ & $0.98(-0.59 ; 2.56)$ & $-1.16(-4.18 ; 1.86)$ & $0.83(-0.74 ; 2.41)$ \\
& & & \\
SES & & & \\
Low & $0.17(-0.77 ; 1.11)$ & $1.49(-0.33 ; 3.32)$ & $0.58(-0.36 ; 1.53)$ \\
Mid & $0.48(-0.46 ; 1.43)$ & $2.80(0.98 ; 4.64)$ & $1.70(0.75 ; 2.65)$ \\
\hline
\end{tabular}


1 8.2 ANEXO B - Artigo modificação de efeito do SES nos

2 desfechos da gravidez

3 TITLE: Individual and contextual Socioeconomical Status as effect

4 modifier in the air pollution-birth outcome association

5 Felipe Parra do Nascimento ${ }^{1, *}$, Marcia Furquim de Almeida ${ }^{2}$,

6 Nelson Gouveia ${ }^{1}$

$7{ }^{1}$ Department of Preventive Medicine, School of Medicine FMUSP,

8 University of São Paulo, SP, Brazil.

$9{ }^{2}$ Department of Epidemiology, University of São Paulo, School of

10 Public Health, São Paulo, Brazil

11 * Corresponding Author - Felipe Parra do Nascimento,

12 Departamento de Medicina Preventiva, Faculdade de Medicina,

13 Universidade de São Paulo, Av. Dr. Arnaldo, n455, São Paulo/SP,

14 Brasil.

15 E-mail: felipe.parra.nascimento@usp.br

16 Submetido na revista International Journal of Epidemiology 
BACKGROUND: Several studies have examined whether air pollution is associated with adverse births outcomes, but it is not clear if socioeconomic status (SES) modifies this relationship.

OBJECTIVES: We investigated if individual and area-level socioeconomic status modified the relationship between ozone, nitrogen dioxide and particulate matter with aerodynamic diameter $<10 \mu \mathrm{m}\left(\mathrm{PM}_{10}\right)$ on preterm deliveries (PTD; gestational age $<37$ weeks) and term low birth weight (TLBW; $<2500 \mathrm{~g}$ ).

METHODS: Analyses were based on almost 1 million singleton live births in São Paulo municipality between 2011 and 2016. Exposure to $\mathrm{PM}_{10}, \mathrm{NO}_{2}$ and $\mathrm{O}_{3}$ were based on date of birth and it was estimated for the entire gestation duration and the trimestral average. The data came from the network of monitoring stations. Multilevel logistic regression models were conducted to examine the effect of air pollutants on both adverse birth outcomes and whether it was modified by SES.

RESULTS: In fully adjusted models, over the entire pregnancy, a $10 \mu \mathrm{g} / \mathrm{m}^{3}$ increase in $\mathrm{O}_{3}$ and $\mathrm{PM}_{10}$ was associated to PTD (odds ratio; $\mathrm{OR}=1.14 \mathrm{Cl} 1.13$, 1.16 and $1.08 \mathrm{Cl}=1.02,1.15$ respectively) and $\mathrm{PM}_{10}$ was associated to TLBW $(\mathrm{OR}=1.08 \mathrm{Cl} 1.03,1.14)$. Associations were modified by individual and arealevel SES for both outcomes. Mothers of low individual SES exposed to $\mathrm{PM}_{10}$ had an additional risk of PTD and TLBW $(\mathrm{OR}=1.04 \mathrm{Cl} 1.04,1.05$ and $1.10 \mathrm{Cl}$ $1.08,1.14$ respectively), while mothers living in low SES areas have an additional risk for TLBW $(O R=1.05 \mathrm{Cl} 1.03,1.06)$. Similar modification effects were found for $\mathrm{O}_{3}$ exposure. Trimester specific associations were weaker but followed a similar pattern.

CONCLUSION: Socioeconomic status modifies the effect of air pollution on adverse birth outcomes. Results indicates that mothers with lower SES may be more susceptible to air pollution effects. 


\section{Introduction}

Adverse birth outcomes such as preterm delivery (PTD, defined as a delivery before 37 weeks of gestation) and low birth weight (LBW, defined as birth weight inferior to $2500 \mathrm{~g}$ ), are considered important epidemiological indicators of newborn health (Stieb et al. 2016). Preterm newborns have the highest infant mortality rates which increases as the gestational age decreases (Mathews and MacDorman 2012) while LBW has been previously associated with a higher risk of metabolic syndrome, type 2 diabetes, cardiovascular diseases and asthma on childhood (Chernausek 2012; Caudri et al. 2007). LBW can be a result of preterm gestations and in term gestations may occur due intrauterine growth restriction which is associated to mother's health condition such as preeclampsia and placental disorders (Laurent et al. 2014, Rich et al., 2009, Wu et al. 2009). It has been reported in previous studies that maternal age, education, income, prenatal care and ethnicity are risk factors for PTD (Yi et al. 2010) and term low birth weight (TLWB, birth weight inferior to $2500 \mathrm{~g}$ on gestations 37 to 42 weeks long) (Gouveia et al. 2004; Romão et al. 2013). The proportion of PTD is increasing in several countries, including Brazil (Alencar et al. 2016) while LBW occurs more frequently in low and middle-income countries (WHO, 2014). In Brazil, LBW presents a stationary trend of about $8 \%$ of live births in the 2011-2015 period (Henriques et al. 2019).

Ambient air pollution has also been consistently associated with adverse birth outcomes. Chronic exposure to air pollution toxic compounds during pregnancy may induce inflammation, affect placental hemodynamics and affect the transport of oxygen and nutrients to the fetus, leading to intrauterine growth restriction and TLBW (Laurent et al. 2014; Li L et al. 2016). Particles of median aerodynamic diameter smaller than $10 \mu \mathrm{m}\left(\mathrm{PM}_{10}\right)$ are composed by many core chemicals that absorbs polycyclic aromatic hydrocarbons (PAH) and other organic compounds, which can generate various forms of inflammations (Delfino et al. 2010) and exposure to them has been associated with a higher risk of PTD (Schifano et al. 2010) and TLBW (Gray et al. 2014, Stieb et al. 2016). Systematic reviews and meta-analyses indicate that this association exist and is biologically plausible (Dadvand et al. 2013; Šrám et al. 2005; Stieb et al. 2012). 
Socioeconomic status (SES) has been reported to modify the effects of exposure to ambient air pollution on adverse birth outcomes, not only at the individual-level, but also at the area-level (Li S et al. 2016). Some studies reported that maternal neighborhood environment has an independent influence on birth outcomes that is not explained by individual SES (O'Neill et al. 2003; Gray et al. 2014). Yorifuji et al. (2013) reported that the occurrence of PDT and TLBW outcomes were associated with lower individual and area level SES in Japan. In the USA, two studies (Li L et al. 2016; Laurent et al. 2014) reported an association of maternal exposure to $\mathrm{NO}_{2}$ with TLBW which was modified by educational level, where mothers with low education were more susceptible to the air pollution effects. A study conducted by Padula and collaborators (2014) found that air pollution generated by traffic increased the chance of PTD with low area-SES acting as an effect modifier, increasing the chance by three times. Erikson et al. (2016), in a study in Canada, reported that neighborhood-level SES and PM2.5 are associated with lower birth weight not only as independent factors but also have an interacting effect, suggesting that some sub-populations can be more vulnerable to air pollution effects. Modification of the association between air pollution $\left(\mathrm{NO}_{2}, \mathrm{CO}\right.$ and $\left.\mathrm{SO}_{2}\right)$ and PTD by area-level SES was also reported in an Australian study (Li S et al., 2016). On the other hand, a study in Italy reported that mothers with high educational level had an increasing risk of PTD associated with $\mathrm{PM}_{10}$ exposure (Schifano et al. 2013).

In the present study, we conducted a population level analysis of all pregnancies in São Paulo municipality over a period of 7 years to evaluate the effect of ambient air pollution on PTD and TLBW and if individual and area level SES modified these associations. Our hypothesis was that outdoor air pollution increases the probability of adverse pregnancy outcomes but it does not affect equally all mothers, with low individual and area-level SES mothers affected the most.

\section{Materials and Methods}

Study area and design: We performed a population-based cross-sectional study in São Paulo municipality, the largest city in Brazil with a population of over 12 
million people in 2017 and a demographic density of 7960 inhabitants/Km² (IBGE, 2017).

Birth data: Information on all live births of São Paulo municipality in the period between January $1^{\text {st }}, 2011$ to December $31^{\text {st }}, 2016$ were collected from birth certificates notified on the Live Births Information System (SINASC) of the Ministry of Health in Brazil. This included maternal information: age, years of education and district of residence; gestation and delivery: type of pregnancy (singleton or multiple), prenatal care (number of attendances during pregnancy: none, 1 to 3 or 4 and more), gestational age (in weeks), and type of delivery (vaginal or cesarean); and newborn: birth date, gender, weight and presence of any kind of congenital anomaly.

Air pollution data: Data were extracted directly from the Companhia Ambiental do Estado de São Paulo CETESB ([2016]), the environmental agency of São Paulo, from January $1^{\text {st }}, 2010$ to December $31^{\text {st }}, 2016$. Air pollution data for 2010 was necessary because the exposure of births on early to mid-2011 began on 2010 . We used the daily mean value for $\mathrm{PM}_{10}$ and $\mathrm{NO}_{2}$ and the highest 8-hour moving average of $\mathrm{O}_{3}$. Daily air pollutant measures were considered valid when at least 16 of the 24-hourly measurements had no errors. CETESB had $13 \mathrm{PM}_{10}, 12 \mathrm{O}_{3}$ and $9 \mathrm{NO}_{2}$ automatic monitoring stations in the 2010-2016 period (Figure 1). Because correlation between monitoring stations values for each pollutant were very high (see Supplementary Material, tables 1 to 3), when data were available for more than one monitoring station, it was calculated as its average. Maternal exposure to air pollution at each trimester of pregnancy was estimated taking into account the length of each gestation and the birth date. Trimestral mean exposure to each pollutant was assigned to each birth. For preterm births, the 3rd trimester exposure was calculated as from the 27th week of pregnancy until delivery.

Meteorological Data: Daily average temperature data was obtained from Instituto de Astronomia, Geofísica, e Ciências Atmosféricas da Universidade de São Paulo monitoring station for January $1^{\text {st }}, 2010$ to December $31^{\text {st }}, 2016$. We 
calculated trimestral mean temperatures for each pregnancy in the same way as for air pollution exposure.

SES data: We used maternal education (years of schooling) as the indicator of individual SES level. This variable was divided into three categories: 0 to 8 years, 9 to 11 years and 12 or more years from the birth certificates. For the area-level SES we built an index using information from the Census 2010 data (http://infocidade.prefeitura.sp.gov.br) for each of the 96 districts of São Paulo. The index was constructed by applying principal components analysis with varimax rotation to the following variables: mean income; literacy rate; oneperson housing; education (mean years of education for residents with 18 years or older); housing conditions (proportion of residences not connected to the sewage network). All variables were first standardized to remove the influence of different units of measurement. The first component explained $65.5 \%$ of the variance and was used to build the socioeconomic index. Each district received a score level based on the socioeconomic index and was ranked and then divided into three tertiles. The 32 highest score districts were allocated to the high SES category, the next 32 to the medium SES and the lowest 32 to the low SES (Figure 1). Results were consistent with previous reports (Takiya et al. 2002). This method has been previously used on other studies with some variations (Blanco-Becerra et al. 2014; Carbajal-Arroyo et al. 2011).

Statistical analysis: First we performed univariate multilevel logistic regression analyses to exam the associations between neighborhood level SES, maternal age, temperature, maternal education, prenatal care, infant's gender and type of delivery and both outcomes. We categorized maternal age into lower than 20 years, 20 to 34 years and older than 34 years. For the multiple multilevel logistic regression, we used hierarchical models with random effects for individual and area-level SES with random intercept for each district area to access the individual and neighborhood effect adjusted for maternal, infant's and pregnancy characteristics using Stata function xtmelogit (Stata 13). Then we tested the interaction between both SES indicators with each pollutant and outcome, adjusted for the same variables. 


\section{Results}

Between 2011 and 2016 there were 1,042,923 live births in São Paulo municipality. We excluded all multiple pregnancies $(27,420)$ those whose deliveries had less than $500 \mathrm{~g}$ or more than $5500 \mathrm{~g}$ (96). We considered that those live births were products of very high-risk pregnancies and air pollution effect would be outweighed by other conditions. We also excluded deliveries with gestational age higher than 42 weeks $(13,867)$ and all births that had any congenital anomalies (17,370). Lastly, we removed all births that had missing data on at least one covariable (4869). The final sample included 979309 births for PTD analysis and 888133 for TLBW analysis.

Overall, the prevalence of PTD was $9.3 \%$ and $3.5 \%$ for TLBW in this population. Mothers were mostly 20 to 34 years old (69.6\%), had 9 to 11 years of education (61\%), lived on low SES districts (55.4\%) and had 4 or more attendances during prenatal care (94.3\%). We observed that prevalence of PTD was higher on male infants while TLBW was higher on females. Younger and older mothers were more likely to have both adverse birth outcomes. SES indicators showed that both low and medium educated mothers and those living on low/medium-SES districts were more likely to experience PTD and TLBW. Cesarean deliveries accounted for more than half of this study deliveries and were unequally distributed through maternal education (34\% low versus $78 \%$ on high) and maternal area-level SES (46\% on low versus $66 \%$ on high) and it was shown to be associated to PDT but not for TLBW. As expected, prenatal care had a large effect on both outcomes as mothers who had less than 4 attendances were more likely to have adverse birth outcomes. Temperature on third trimester was positively associated to PTD (data not shown). Full descriptive statistics for those characteristics are shown on Table 1 as well as univariate odds ratios for simple multilevel logistic regressions.

Table 2 shows summary statistics for temperature and air pollution levels in São Paulo during the 2010-2016 period. Mean values for $\mathrm{O}_{3}$ were near 65 $\mu \mathrm{g} / \mathrm{m}^{3}$ while $\mathrm{PM}_{10}$ and $\mathrm{NO}_{2}$ were 34 and $70 \mu \mathrm{g} / \mathrm{m}^{3}$ respectively while mean ambient temperature was around $20^{\circ} \mathrm{C}$. Correlation between $\mathrm{NO}_{2}$ and $\mathrm{PM}_{10}$ levels 
were somewhat high (Spearman's $r=0.796$ ) and average between $\mathrm{O}_{3}$ and temperature $(r=0.569$, Supplementary Material table 4$)$. By analyzing the correlations between each pollutant trimestral exposure and entire pregnancy, we found that entire pregnancy exposure was more correlated with the $2^{\text {nd }}$ trimester exposure $\left(r=0.774\right.$ for $\left.\mathrm{PM}_{10}\right)$. Temperature, on the other hand, had a negative correlation with both $\mathrm{PM}_{10}$ and $\mathrm{NO}_{2}$ levels (Supplementary Material, table 5).

Multiple multilevel logistic regression analysis evaluated the effect of exposure to pollutants over the whole gestational period and each trimester separately, adjusting by infant's gender, maternal age, maternal education, arealevel SES, type of delivery, prenatal care and respective mean temperature in the period. For a $10 \mu \mathrm{g} / \mathrm{m}^{3}$ increase in mean $\mathrm{PM}_{10}$ levels on 1st, 2nd, 3rd trimesters and entire pregnancy, we found a statistically significant increase in TLBW risk. $\mathrm{NO}_{2}$ levels during 1st and 2nd trimesters and entire pregnancy were also positively associated with TLBW. PTD risk was associated with an increase in 10 $\mu \mathrm{g} / \mathrm{m}^{3}$ in $\mathrm{O}_{3}$ levels during $2 \mathrm{nd}$ and 3rd trimesters and entire pregnancy and also with $\mathrm{PM}_{10}$ levels for 1 st trimester and entire pregnancy (Table 3).

When evaluating the effect modification by SES, we found that lower maternal education lead to an additional risk of PTD and TLBW for all pollutants and on all trimesters and whole gestation periods. Mothers with 9 to 11 years of study also showed higher risk of PTD in comparison to mothers that had 12 years or more associated with exposure to $\mathrm{O}_{3}$ on the 3rd trimester and with $\mathrm{PM}_{10}$ in all periods. Regarding area-level SES, mothers living in low and medium SES districts showed higher risk of TLBW associated with exposure to all pollutants on all periods while the effect modification was not consistent for PTD (Figure 2).

\section{Discussion}

In this study we evaluated if individual and area-level SES modified the association between air pollution and PTD and TLBW. We report that mothers with lower educational level (individual SES) and living on poorer districts (area- 
level SES) were more likely to experience PTD or TLBW in relation to mothers with high literacy and living on high-SES districts, after controlling for temperature, maternal age, newborn gender, type of delivery and number of prenatal care visits. Our results show that both individual and contextual SES are associated to a higher chance of PTD and even higher for TLBW. In addition, we observed that for TLBW both SES indicators interacted with exposure to air pollutants in all periods of gestation. These findings indicate that low SES mothers have an additional risk of an adverse pregnancy outcome.

Other studies have found similar results concerning the interaction effect of air pollution and area-level SES. Yi et al. (2010), in South Korea, reported that an increase of $10 \mathrm{\mu g} / \mathrm{m}^{3}$ in exposure to $\mathrm{PM}_{10}$ during the $2 \mathrm{nd}$ trimester of gestation lead to an additional increase of $3.12 \%$ in the probability of PTD $(95 \% \mathrm{Cl}, 0.17-$ $6.15 \%)$ for mothers living on low-income districts. Padula et al. (2014), in the USA, also reported a similar effect on PTD associated with an increase of 42.77 $\mu \mathrm{g} / \mathrm{m}^{3}$ in the exposure to $P M_{10}$ in the second trimester of gestation $(O R=1.13$, $\mathrm{Cl} 95 \%=1.06-1.22)$ and also over the entire gestation period $(\mathrm{OR}=1.13, \mathrm{Cl} 95 \%$ $=1.05-1.21$ ) on mothers living on low SES neighborhoods. On the other hand, also in Canada, Stieb et al. (2016) reported that the association between $\mathrm{PM}_{2.5}$ and TLBW was not sensitive to neighborhood SES adjustment.

Gray et al. (2014), in the USA, reported that the effect of PM2.5 on PTD and LBW was modified by individual (maternal education) and area-level (census based median household income) SES. They reported that mothers with less than 12 years of study had a higher risk of both LBW and PTD (OR $=1.14-1.25$ for LBW and $1.05-1.12$ for PTD) and mothers living on low median income household also had increased chance of LBW outcomes $(O R=1.00-1.08)$ but not for PTD $(\mathrm{OR}=0.98-1.04)$.

Differences between the results reported in this study and others previously published could be due to differences on air pollution exposure assessment, sample size or area-level SES definition. We found some inverse associations for $\mathrm{NO}_{2}$ exposure on 1 st and 2nd trimesters and PTD. Padula et al. (2014) hypothesized that these inverse associations might be due to selection bias where only live births are included. Therefore, if $\mathrm{NO}_{2}$ exposure has a stronger association with stillbirth than with PTD on the beginning of the gestations, we 
are examining only those who had survived the period when the mortality would be higher. Future studies should investigate further the association between ambient air pollution and stillbirth.

Higher maternal education may indicate a better understanding of the adverse effects of multiple environmental agents, healthier lifestyle and greater access to health care services which could reduce the impact of air pollution on pregnancy outcomes (Šrám et al. 2005) while lower educated mothers are more likely to have a poor health care, inadequate nutrition and non-healthy behaviors such as smoking (O'Neill et al., 2008), increasing their vulnerability to air pollution, and consequently, the risk of adverse pregnancy outcomes.

Regarding maternal, gestational and newborn characteristics, our results were similar to those previously reported by Gouveia et al. (2004). Just like the present study, female gender, higher maternal age, low maternal education, low number of prenatal care visits and normal delivery were associated to TLBW in their study. The difference was that the present study reports that mothers younger than 20 years are also a risk group for TLBW. In general, our findings are also in agreement with international studies (Morello-Frosch et al. 2010; Yorifuji et al. 2013)

Our study had several strengths such as the large number of deliveries, municipal multiyear data from birth certificates and residential information and a robust statistical model, but it also had some limitations. We have not been able to measure the exact exposure to air pollution for each pregnancy because maternal residential address at time of birth may not be the same the mother had during her gestation and we also had no information about maternal mobility during pregnancy, which could have led to a non-differential exposure misclassification. This implies that the magnitude of our results might be underestimated. Moreover, we could not control maternal smoking habit, alcohol consumption, nutritional status and drug ingestion although smoking, alcohol and drug ingestion may have been indirectly adjusted by educational level (O'Neill et al. 2003; Barbieri et al., 2000). In addition, we had no information about maternal morbidities such as diabetes or hypertension which can potentially lead to adverse gestation outcomes (Yorifuji et al. 2013; Padula et al. 2014). Birth 
295 certificates did not indicate if the PTD were spontaneous or indicated. Finally, we 296 could not control the spatial heterogeneity that districts have.

297 In conclusion, higher exposure to $\mathrm{O}_{3}$ over the entire pregnancy increased 298 the chances of PTD in 14\% and PM10 exposure increased the chances of both 299 PTD and TLBW in 8\%. Also, considering the entire pregnancy period, low 300 education mothers had an additional $10 \%$ risk of TLBW due to $\mathrm{PM}_{10}$ and those 301 living on low SES districts had an additional 14\% risk of PTD due to $\mathrm{O}_{3}$ exposure. 302 These results provide further evidence that individual and area-level SES 303 modifies the effect of air pollution on PTD and TLBW.

304

305 Conflicts of interest

306 The authors have no conflicts of interest to disclose.

307

308

Acknowledgments

309

This study was financed in part by the Coordenação de Aperfeiçoamento 310 de Pessoal de Nível Superior - Brasil (CAPES) - Finance Code 001. 


\section{References}

313 Alencar GP, Silva ZP, Santos PC, Raspantini PR, Moura BLA, Almeida MF, et al. What is the impact of interventions that prevent fetal mortality on the increase of preterm live births in the State of São Paulo, Brazil? BMC Pregnancy \& Childbirth. $2015 ; 15: 152$.

Barbieri MA, Silva AA, Bettiol $\mathrm{H}$, et al. Risk factors for the increasing trend in low birth weight among live births born by vaginal delivery, Brazil. Rev Saude Publica. 2000; 34:596-602.

Blanco-Becerra LC, Miranda-Soberanis V, Barraza-Villareal A, Junger W, Hurtado-Dias M, Romieu I. Effect of socioeconomic status on the association between air pollution and mortality in Bogota, Colombia. Salud Publica Mex. 2014;56(4):371-8.

Carbajal-Arroyo L, Miranda-Soberanis V, Medina-Ramón M, Rojas-Bracho L, Tzintzun G, Solís-Gutiérrez $\mathrm{P}$, et al. Effect of $\mathrm{PM}_{10}$ and $\mathrm{O}_{3}$ on infant mortality among residents in the Mexico City Metropolitan Area: a case-crossover analysis, 1997-2005. J Epidemiol Community Health. 2011;65(8):715-21.

Caudri D, Wijga A, Gehrign U, Smit HA, Brunekreef B, Kerkhof M, et al. 330 Respiratory symptoms in the first 7 years of life and birth weight at term: the PIAMA Birth Cohort. Am J Respir Care Med. 2007;175(10):1078-85.

CETESB - Companhia Ambiental do Estado de São Paulo. Qualar - Sistema de informações da qualidade do ar [Internet]. São Paulo: CETESB; [2016] [cited 2017 Sep 25]. Available from: http://qualar.cetesb.sp.gov.br/qualar/home.do

Chernausek SD. Update: consequences of abnormal fetal growth. J Clin Endocrinol Metab. 2012; 97(3): 689-95. 
341 Evaluation of Effect and Heterogeneity. Environ Health Perspect. 2013:121(3); $342 \quad 367-373$.

343 Delfino RJ, Staimer N, Tjoa T, Arhami M, Polodori A, Gillen DL, et al. Associations 344 of primary and secondary organic aerosols with airway and systemic 345 inflammation in an elderly panel cohort. Epidemiology. 2010; 21(6): 892-902.

346 Erickson AC, Ostry A, Chan LHM, Arbour L. The reduction of birth weight by fine 347 particulate matter and its modification by maternal and neighbourhood-level 348 factors: a multilevel analysis in British Columbia, Canada. Environmental Health. $3492016 ; 15: 51$.

350 Gouveia N, Bremmer SA, Novaes HMD. Association between ambient air 351 pollution and birth weight in São Paulo, Brazil. J Epidemiol Community Health. 352 2004;58: 11-17.

353 Gray SC, Edwards SE, Schultz BD, et al. Assessing the impact of race, social 354 factors and air pollution on birth outcomes: a population-based study. Environ 355 Health. 2014; 13:4.

356 Henriques LB, Alves EB, Vieira FMSB, Cardoso BB, D’Angeles ACR, Cruz OG, 357 et al. Acurácia da determinação da idade gestacional no Sistema de Informações 358 sobre Nascidos Vivos (SINASC): um estudo de base populacional. Cad de Saúde 359 Pública [Internet]. 2019 [cited 2020 Apr];35(3):e00098918. Available from: 360 http://www.scielo.br/scielo.php?script=sci_arttext\&pid=S0102-

$361311 \times 2019000305011$ Instituto Brasileiro de Geografia e Estatística [Internet]. Rio de Janeiro: IBGE Instituto Brasileiro de Geografia e Estatística; c2017 [cited 2018 May 10]. São Paulo [municipality]: panorama. Available from:

365 https://cidades.ibge.gov.br/brasil/sp/sao-paulo/panorama

366 Laurent O, Hu J, Li L, Cockburn M, Escobedo L, Kleeman BJ, et al. Sources of 367 contents of air pollution affecting term low birth weight in Los Angeles County, 368 California, 2001-2008. Environ Res. 2014; 134; 488-95. 
369

370

371

372

Li L, Laurent O, Wu J. Spatial variability of the effect of air pollution on term birth weight: evaluating influential factors using a Bayesian hierarchical models. Environ Health. 2016; 15:14.

Li S, Guo Y, Williams G. Acute Impact of Hourly Ambient Air Pollution on Preterm Birth. Environ Health Perspect. 2016; 124(10): 1623-9.

Mathews JM, MacDorman MF. Infant mortality statistics from 2008 period linked birth/infant death data set national. Natl Vial Stat Rep. 2012; 60(5):1-27.

Morello-Frosch R, Jesdale BM, Sadd JL, Pastor M. Ambient Air Pollution and fullterm birth weight in California. Environ Health. 2010; 9:44.

O'Neill MS, Bell ML, Ranjit N, Cifuentes LA, Loomis D, Gouveia N, et al. Air pollution and mortality in Latin America: the role of education. Epidemiology. 2008;19(6):810-9.

O'Neill MS, Jerett M, Kawachi I, Levy JI, Cohen AJ, Gouveia N, et al. Health, Wealth, and Air Pollution: Advancing Theories and Methods. Environ Health Perspect. 2003;111(16):1861-70.

Padula AM, Mortimer KM, Tager IB, Hammond K, Lurmann FW, Yang W, et al. Traffic-related air pollution and risk of preterm birth in the San Joaquin Valley of California. Ann Epidemiol. 2014;24(12):888-95.

Rich DQ, Demissie K, Lu SE, Kamat L, Wartenberg D, Rhoads GG. Ambient air pollutant concentrations during pregnancy and the risk of fetal growth restriction. J Epidemiol Community Health. 2009; 63:488-96.

Romão R, Pereira LAA, Saldiva PHN, Pinheiro PM, Braga ALF, Martins LC. The relationship between low birth weight and exposure to inhalable particulate matter. Cad Saude Publica. 2013; 29(6) 1101-08.

Schifano P, Lallo A, Asta F, Sario M, Davoli M, Michelozzi P. Effect of ambient temperature and air pollutants on the risk of preterm birth, Rome 2001-2010. Environ Int. 2013; 61:77-87. 
396 Šrám JR, Binková B, Dejmek J, Bobak M. Ambient Air Pollution and Pregnancy 397 Outcomes: A Review of the Literature. Environ Health Perspect. 2005: 113;375$398 \quad 82$.

399 StataCorp, LP 4905 Lakeway Drive, College Station, TX 77845, USA.

400 Stieb DM, Chen L, Eshoul M, Judek S. Ambient air pollution, birth weight and 401 preterm birth: a systematic review and meta-analysis. Environ Res. 2012: $402 \quad 117 ; 100-111$.

403 Stieb DM, Chen L, Beckerman BS, Jerret M, Crouse DL, Omariba DWR, et al. 404 Associations of Pregnancy Outcomes and PM2.5 in a National Canadian Study. Environ Health Perspect. 2016; 124(2): 243-9.

Takiya H. Atlas Ambiental do Município de São Paulo: fase I: diagnóstico e bases para a definição de políticas públicas para as áreas verdes no município de São Paulo. São Paulo: Prefeitura do Município de São Paulo. Secretaria Municipal do Meio Ambiente, Secretaria Municipal de Planejamento Urbano; 2002 Jul. Relatório final. Processo no: 1999/10955-9. Projeto Biota - FAPESP.

411 World Health Organization (WHO). Global nutrition targets 2025: low birth weight 412 policy brief [Internet]. Geneva: WHO; 2014 [cited 2019 Oct 22]. Available from: 413 http://apps.who.int/iris/bitstream/10665/149020/2/WHO_NMH_NHD_14.5_eng.p 414 df?ua=1

415 Wu J, Ren C, Delfino RJ, Chung J, Wilhelm M, Ritz V. Association between local 416 traffic-generated air pollution and preeclampsia and preterm delivery in the south 417 Coast air basin of California. Environ Health Perspect. 2009; 177:1773-79.

418 Yi Okhee, Kim H, Ha E. Does area level socioeconomic status modify the effects 419 of $\mathrm{PM}_{10}$ on preterm delivery? Environ Res. 2010; 110: 55-61.

420 Yorifuji T, Naruse H, Kashima S, Takao S, Murakoshi T, Doi H, et al. Residential 421 proximity to major roads and adverse birth outcomes: a hospital-based study. 422 Environ Health. 2013;12:34. 
Table 1. Descriptive statistics and Odds Ratios $(95 \% \mathrm{Cl})$ from simple multilevel logistic regression for the study population, Sao Paulo, 2011-2016

\begin{tabular}{|c|c|c|c|c|c|c|}
\hline Variables & Births $n=979306$ & PTD n(\%) & $\begin{array}{l}\text { Odds Ratio } \\
(95 \% \mathrm{Cl})^{a}\end{array}$ & $\begin{array}{l}\text { Term births } \\
\mathrm{n}=888133\end{array}$ & TLBW n(\%) & $\begin{array}{l}\text { Odds Ratio } \\
(95 \% \mathrm{Cl})^{\mathrm{a}}\end{array}$ \\
\hline \multicolumn{7}{|l|}{ Gender } \\
\hline Female $^{b}$ & 478635 (48.8\%) & 42528 (8.9) & \multirow{2}{*}{$\begin{array}{c}1.00 \\
1.10(1.09,1.11)\end{array}$} & 436107 (49.1\%) & $18506(4.2)$ & \multirow{2}{*}{$\begin{array}{c}1.00 \\
0.67(0.65,0.69)\end{array}$} \\
\hline Male & $500671(51.2 \%)$ & $48645(9.7)$ & & 452026 (50.9\%) & $13080(2.9)$ & \\
\hline \multicolumn{7}{|l|}{ Maternal Age } \\
\hline$<20$ & $129461(13.2 \%)$ & $14429(11.1)$ & $1.32(1.29,1.35)$ & $115032(23.0 \%)$ & $5012(4.4)$ & \multirow{3}{*}{$\begin{array}{c}1.00 \\
1.11(1.07,1.14)\end{array}$} \\
\hline $20-34^{b}$ & $682131(69.6 \%)$ & $59127(8.7)$ & \multirow{2}{*}{$\begin{array}{c}1.00 \\
1.24(1.21,1.26)\end{array}$} & $623004(70.1 \%)$ & 20988 (3.4) & \\
\hline$>34$ & $167714(17.2 \%)$ & $17617(10.5)$ & & 150097 (16.9\%) & $5586(3.7)$ & \\
\hline \multicolumn{7}{|l|}{ Maternal Education } \\
\hline $12+$ years $^{b}$ & 260412 (26.6\%) & $22973(8.8)$ & \multirow{2}{*}{$\begin{array}{c}1.00 \\
1.06(1.04,1.08)\end{array}$} & 237439 (26.7\%) & $6766(2.8)$ & \multirow{3}{*}{$\begin{array}{c}1.00 \\
1.28(1.24,1.31) \\
1.73(1.67,1.80)\end{array}$} \\
\hline 9 to 11 years & $597716(61.0 \%)$ & $55734(9.3)$ & & $541982(61.0 \%)$ & $19554(3.6)$ & \\
\hline 0 to 8 years & $121178(22.4 \%)$ & $12466(10.3)$ & $1.18(1.16,1.21)$ & $108712(12.3 \%)$ & $5266(4.8)$ & \\
\hline \multicolumn{7}{|l|}{ SES } \\
\hline High b $^{b}$ & 168955 (17.3\%) & $15014(8.9)$ & \multirow{3}{*}{$\begin{array}{c}1.00 \\
1.07(1.04,1.09) \\
1.06(1.04,1.08)\end{array}$} & 153941 (17.3\%) & $4507(2.9)$ & \multirow{3}{*}{$\begin{array}{c}1.00 \\
1.16(1.12,1.21) \\
1.32(1.28,1.37)\end{array}$} \\
\hline Medium & 267649 (27.3\%) & $25274(9.4)$ & & $242375(27.3 \%)$ & $8218(3.4)$ & \\
\hline Low & $542702(55.4 \%)$ & $50885(9.4)$ & & 491817 (55.4\%) & $18861(3.8)$ & \\
\hline \multicolumn{7}{|l|}{ Type of Delivery } \\
\hline Vaginal $^{b}$ & $461010(47.1 \%)$ & $41905(9.1)$ & \multirow{2}{*}{$\begin{array}{c}1.00 \\
1.05(1.03,1.06)\end{array}$} & 419105 (47.2\%) & 15167 (3.6) & \multirow{2}{*}{$\begin{array}{c}1.00 \\
0.97(0.94,0.99)\end{array}$} \\
\hline Cesarean & 518296 (52.9\%) & $49268(9.5)$ & & $469028(52.8 \%)$ & 16419 (3.5) & \\
\hline \multicolumn{7}{|l|}{ Prenatal Care } \\
\hline 4 or more ${ }^{b}$ & 923429 (94.3\%) & $78252(8.5)$ & \multirow{3}{*}{$\begin{array}{c}1.00 \\
3.23(3.16,3.31) \\
3.30(3.17,3.44)\end{array}$} & 845177 (95.2\%) & $28431(3.4)$ & \multirow{3}{*}{$\begin{array}{c}1.00 \\
1.98(1.89,2.07) \\
3.33(3.12,3.56)\end{array}$} \\
\hline 1 to 3 & 43224 (4.4\%) & $9957(23.0)$ & & 33267 (3.7\%) & $2148(6.5)$ & \\
\hline None & $12653(1.3 \%)$ & $2964(23.4)$ & & $9689(1.1 \%)$ & $1007(10.4)$ & \\
\hline
\end{tabular}

a Odds Ratio from simple multilevel logistic regression

b Reference category 
Table 2: Summary statistics of daily air pollution and temperature from 20112016, São Paulo, Brazil

\begin{tabular}{cccccc}
\hline Variable & Minimum & 25th & Median & 75th & Maximum \\
\hline $\mathrm{O}_{3}\left(\mu \mathrm{g} / \mathrm{m}^{3}\right)$ & 3.67 & 44.50 & 64.67 & 89.00 & 187.00 \\
$\mathrm{PM}_{10}\left(\mu \mathrm{g} / \mathrm{m}^{3}\right)$ & 9.50 & 25.67 & 34.00 & 45.00 & 110.67 \\
$\mathrm{NO}_{2}\left(\mu \mathrm{g} / \mathrm{m}^{3}\right)$ & 10.00 & 52.00 & 72.00 & 97.00 & 209.00 \\
Temperature $\left({ }^{\circ} \mathrm{C}\right)$ & 7.25 & 17.40 & 19.86 & 22.35 & 28.05 \\
\hline
\end{tabular}

Table 3: Odds Ratios $(95 \% \mathrm{Cl})$ from multilevel logistic regression models, adjusted by covariates, Sao Paulo, 2011-2016

\begin{tabular}{lccccc}
\hline Outcome & Pollutant & 1st Trimester & 2nd Trimester & 3rd Trimester & $\begin{array}{l}\text { Entire } \\
\text { Pregnancy }\end{array}$ \\
& & & & & \\
\hline \multirow{3}{*}{$\mathrm{PTD}$} & $\mathrm{NO}_{3}$ & $0.99(0.98,1.00)$ & $1.06(1.05,1.06)$ & $1.03(1.02,1.04)$ & $1.14(1.13,1.16)$ \\
& $\mathrm{NM}_{10}$ & $1.02(1.01,1.03)$ & $1.01(1.00,1.02)$ & $0.98(0.95,1.01)$ & $1.08(1.02,1.15)$ \\
& $\mathrm{O}_{3}$ & $0.99(0.98,1.00)$ & $1.00(0.99,1.01)$ & $1.01(1.00,1.02)$ & $1.00(0.98,1.02)$ \\
& $\mathrm{NO}_{2}$ & $1.01(1.00,1.02)$ & $1.01(1.00,1.02)$ & $1.00(0.99,1.01)$ & $1.01(1.00,1.02)$ \\
& $\mathrm{PM}_{10}$ & $1.03(1.01,1.07)$ & $1.02(1.01,1.04)$ & $1.04(1.01,1.07)$ & $1.08(1.03,1.14)$ \\
& & & & &
\end{tabular}


Figure 1 - Map of São Paulo municipality showing the distribution of air quality monitoring stations and different SES by district:

Figure 2 - Effect Modification of individual and area-SES on PTD and TLBW risk associated to air pollutants exposure*:

*Footnote: upper-left figure is the effect modification of education X PTD, upperright area-level SES X PTD, lower left education X TLBW, lower-right area-level SES X TLBW. 
Figure 1 - São Paulo municipality Spatial distribution according to census tracts:

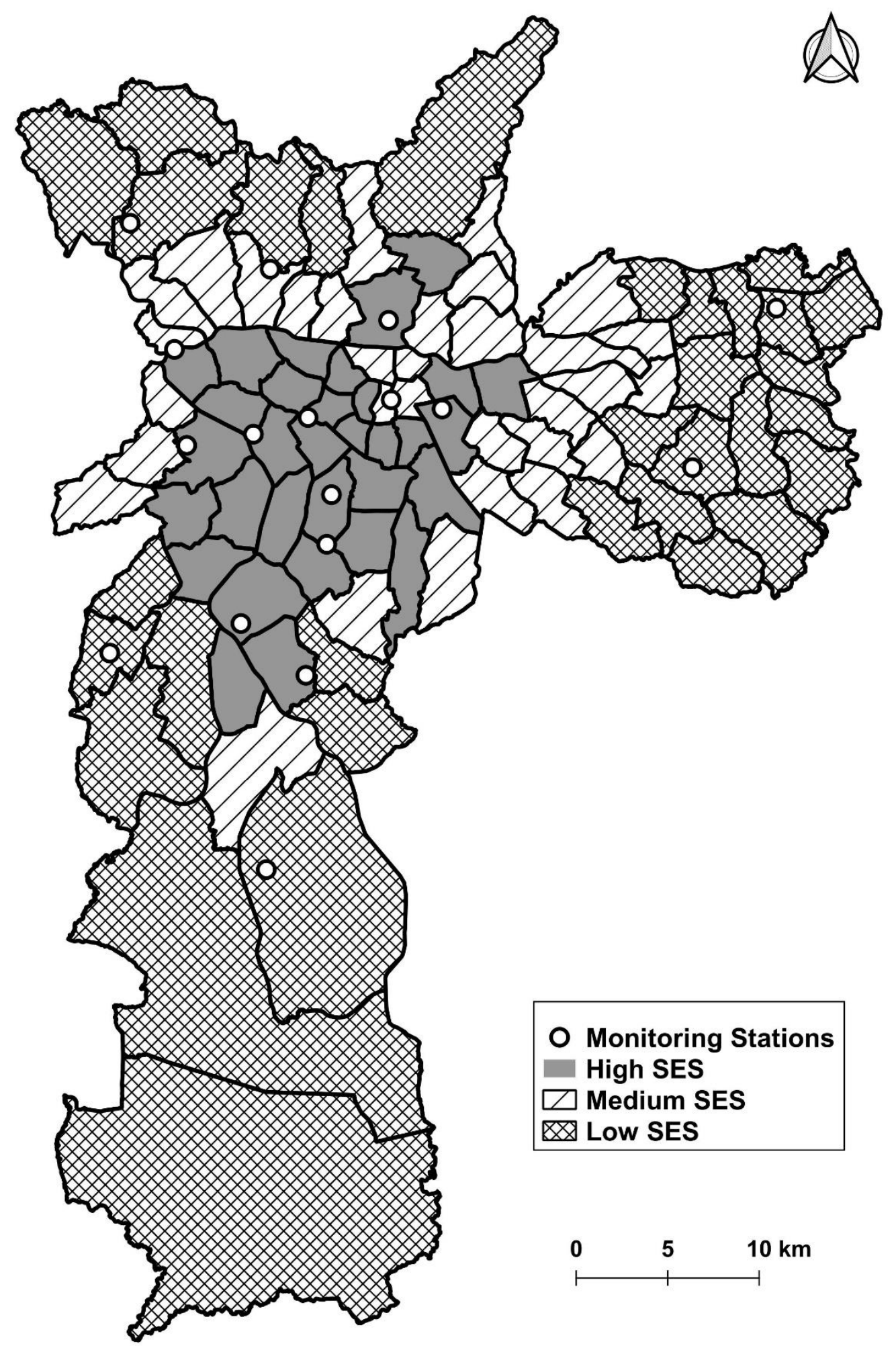



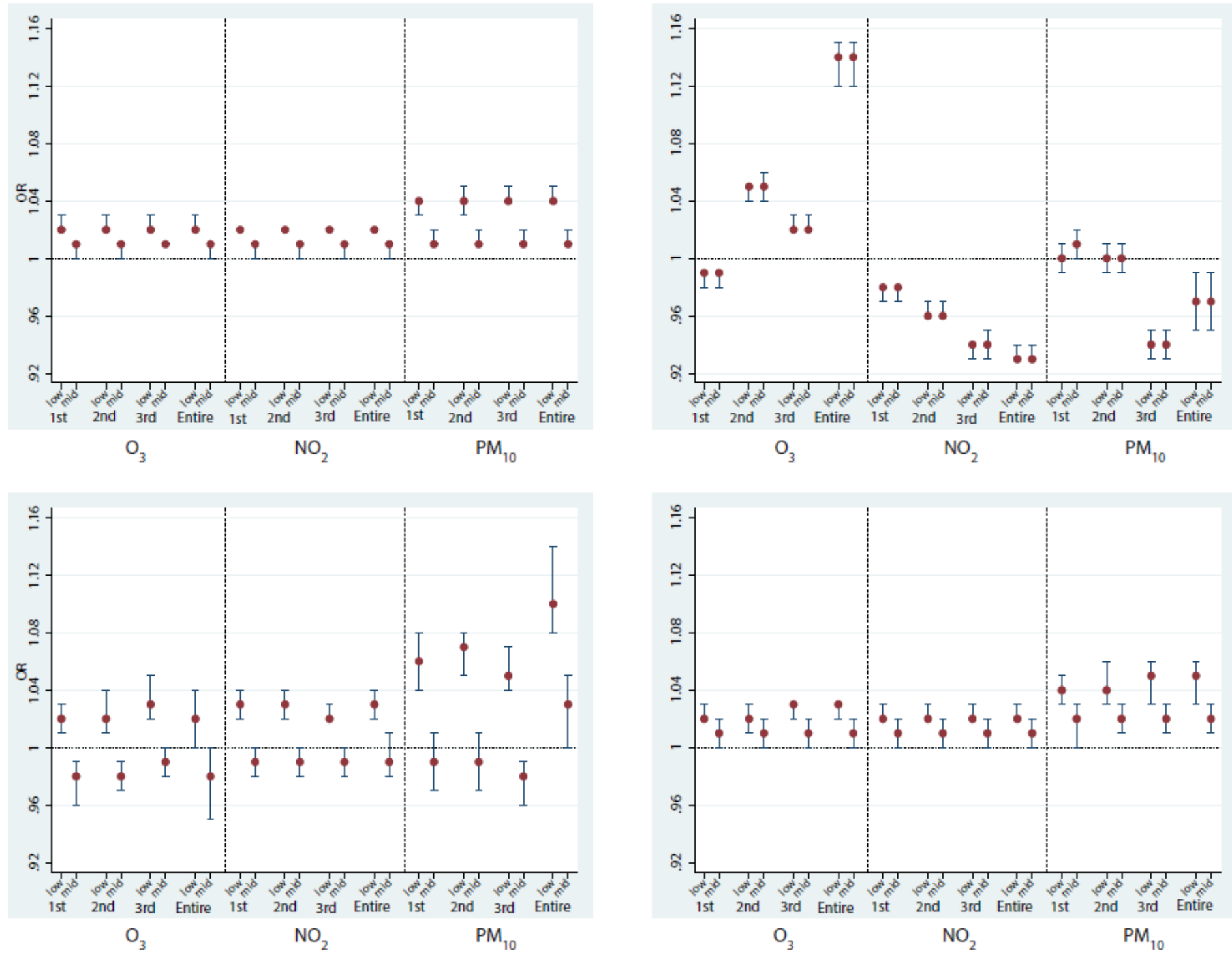
Table S1: Correlation between Monitoring Stations $\mathrm{PM}_{10}$ Levels

\begin{tabular}{|c|c|c|c|c|c|c|c|c|c|c|c|c|c|}
\hline Monitoring & & Pq D. & & & & Cerqueira & N Sra & & Santo & & Marg & Capão & \\
\hline Stations & Santana & P II & Congonhas & Ibirapuera & Mooca & Cesar & do Ó & Parelheiros & Amaro & Interlagos & Tiete & Redondo & Pinheiros \\
\hline Pq D. P II & 0.785 & & & & & & & & & & & & \\
\hline Congonhas & 0.831 & 0.809 & & & & & & & & & & & \\
\hline Ibirapuera & 0.800 & 0.809 & 0.792 & & & & & & & & & & \\
\hline Mooca & 0.835 & 0.771 & 0.846 & 0.766 & & & & & & & & & \\
\hline \multicolumn{14}{|l|}{ Cerqueira } \\
\hline Cesar & 0.843 & 0.792 & 0.860 & 0.760 & 0.859 & & & & & & & & \\
\hline N Sra do Ó & 0.757 & 0.709 & 0.760 & 0.679 & 0.737 & 0.718 & & & & & & & \\
\hline $\begin{array}{l}\text { Parelheiros } \\
\text { Santo }\end{array}$ & 0.533 & 0.486 & 0.545 & 0.591 & 0.553 & 0.648 & 0.444 & & & & & & \\
\hline Amaro & 0.829 & 0.750 & 0.842 & 0.862 & 0.852 & 0.902 & 0.670 & 0.593 & & & & & \\
\hline Interlagos & 0.845 & 0.788 & 0.873 & 0.862 & 0.882 & 0.893 & 0.713 & 0.573 & 0.900 & & & & \\
\hline $\begin{array}{l}\text { Marg Tiete } \\
\text { Capão }\end{array}$ & 0.618 & 0.521 & 0.652 & 0.399 & 0.618 & 0.758 & 0.615 & 0.656 & 0.651 & 0.626 & & & \\
\hline Redondo & 0.794 & 0.712 & 0.799 & 0.876 & 0.828 & 0.873 & 0.806 & 0.596 & 0.869 & 0.895 & 0.672 & & \\
\hline $\begin{array}{l}\text { Pinheiros } \\
\text { Itaim }\end{array}$ & 0.804 & 0.838 & 0.873 & $*$ & 0.832 & 0.889 & 0.812 & 0.593 & 0.846 & 0.862 & 0.740 & 0.834 & \\
\hline Paulista & 0.856 & 0.715 & 0.859 & $*$ & 0.877 & 0.894 & 0.843 & 0.604 & 0.845 & 0.842 & 0.729 & 0.816 & 0.845 \\
\hline
\end{tabular}


Table S2: Correlation between Monitoring Stations $\mathrm{O}_{3}$ Levels

\begin{tabular}{|c|c|c|c|c|c|c|c|c|c|c|c|c|}
\hline Monitoring & & Santo & & & & & N Sra do & & & & & Itaim \\
\hline Stations & Santana & Amaro & Pq D P II & Ibirapuera & Mooca & Ipen & Ó & Itaquera & Parelheiros & Pinheiros & Interlagos & Paulista \\
\hline Santo & & & & & & & & & & & & \\
\hline Amaro & 0.865 & & & & & & & & & & & \\
\hline Pq D P II & 0.896 & 0.877 & & & & & & & & & & \\
\hline Ibirapuera & 0.864 & 0.914 & 0.888 & & & & & & & & & \\
\hline Mooca & 0.864 & 0.863 & 0.872 & 0.863 & & & & & & & & \\
\hline Ipen & 0.875 & 0.936 & 0.876 & 0.904 & 0.840 & & & & & & & \\
\hline N Sra do Ó & 0.947 & 0.846 & 0.871 & 0.851 & 0.838 & 0.862 & & & & & & \\
\hline Itaquera & 0.818 & 0.811 & 0.773 & 0.827 & 0.806 & 0.816 & 0.808 & & & & & \\
\hline Parelheiros & 0.771 & 0.914 & 0.791 & 0.821 & 0.805 & 0.820 & 0.763 & 0.792 & & & & \\
\hline Pinheiros & 0.786 & 0.880 & 0.846 & 0.877 & 0.812 & 0.882 & 0.784 & 0.742 & 0.744 & & & \\
\hline $\begin{array}{l}\text { Interlagos } \\
\text { Itaim }\end{array}$ & 0.874 & 0.939 & 0.846 & 0.902 & 0.830 & 0.902 & 0.872 & 0.848 & 0.894 & 0.822 & & \\
\hline $\begin{array}{l}\text { Paulista } \\
\text { Capão }\end{array}$ & 0.885 & 0.843 & 0.849 & 0.845 & 0.865 & 0.837 & 0.860 & 0.870 & 0.795 & 0.792 & 0.846 & \\
\hline Redondo & 0.855 & 0.915 & 0.824 & 0.873 & 0.843 & 0.890 & 0.853 & 0.763 & 0.837 & 0.827 & 0.917 & 0.803 \\
\hline
\end{tabular}


Table S3: Correlation between Monitoring Stations $\mathrm{NO}_{2}$ Levels

\begin{tabular}{|c|c|c|c|c|c|c|c|c|c|}
\hline & Pq d Pedro II & Congonhas & Ibirapuera & $\begin{array}{l}\text { Cerqueira } \\
\text { Cesar }\end{array}$ & Ipen & Parelheiros & Pinheiros & Interlagos & $\begin{array}{l}\text { Capão } \\
\text { Redondo }\end{array}$ \\
\hline Congonhas & 0.752 & & & & & & & & \\
\hline Ibirapuera & 0.882 & 0.782 & & & & & & & \\
\hline \multicolumn{10}{|l|}{ Cerqueira } \\
\hline Cesar & 0.868 & 0.812 & 0.869 & & & & & & \\
\hline Ipen & 0.837 & 0.730 & 0.832 & 0.833 & & & & & \\
\hline Parelheiros & 0.724 & 0.648 & 0.730 & 0.779 & 0.750 & & & & \\
\hline Pinheiros & 0.822 & 0.778 & 0.832 & 0.869 & 0.824 & 0.746 & & & \\
\hline Interlagos & 0.808 & 0.690 & 0.847 & 0.829 & 0.822 & 0.857 & 0.820 & & \\
\hline \multicolumn{10}{|l|}{ Capão } \\
\hline Redondo & 0.831 & 0.686 & 0.811 & 0.832 & 0.857 & 0.876 & 0.805 & 0.902 & \\
\hline Marg Tiete & 0.818 & 0.781 & 0.792 & 0.883 & 0.782 & 0.806 & 0.846 & 0.819 & 0.831 \\
\hline
\end{tabular}

Table S4: Correlation between pollutants and temperature

\begin{tabular}{llll}
\hline & $\mathrm{O}_{3}$ & $\mathrm{PM}_{10}$ & $\mathrm{NO}_{2}$ \\
\hline $\mathrm{PM}_{10}$ & 0.446 & & \\
$\mathrm{NO}_{2}$ & 0.416 & 0.796 & \\
Temperature & 0.569 & 0.094 & 0.173 \\
\hline
\end{tabular}


Table S5: Correlation between pollutants and temperature over each exposure period

\begin{tabular}{|c|c|c|c|c|c|c|c|c|c|c|c|c|c|c|c|}
\hline $\begin{array}{l}\text { Pearson } \\
\text { Correlation }\end{array}$ & $\begin{array}{l}\mathrm{O}_{3} \\
\text { Entire }\end{array}$ & $\begin{array}{l}\mathrm{O}_{3} \\
\text { Trimester } \\
1\end{array}$ & $\begin{array}{l}\mathrm{O}_{3} \\
\text { Trimester } \\
2\end{array}$ & $\begin{array}{l}\mathrm{O}_{3} \\
\text { Trimester } \\
3\end{array}$ & $\begin{array}{l}\mathrm{PM}_{10} \\
\text { Entire }\end{array}$ & $\begin{array}{l}\mathrm{PM}_{10} \\
\text { Trimester } \\
1\end{array}$ & $\begin{array}{l}\mathrm{PM}_{10} \\
\text { Trimester } \\
2\end{array}$ & $\begin{array}{l}\mathrm{PM}_{10} \\
\text { Trimester } \\
3\end{array}$ & $\begin{array}{l}\mathrm{NO}_{2} \\
\text { Entire }\end{array}$ & $\begin{array}{l}\mathrm{NO}_{2} \\
\text { Trimester } \\
1\end{array}$ & $\begin{array}{l}\mathrm{NO}_{2} \\
\text { Trimester } \\
2\end{array}$ & $\begin{array}{l}\mathrm{NO}_{2} \\
\text { Trimester } \\
3\end{array}$ & $\begin{array}{l}\text { Temp } \\
\text { Entire }\end{array}$ & $\begin{array}{l}\text { Temp } \\
\text { Trimester } \\
1\end{array}$ & $\begin{array}{l}\text { Temp } \\
\text { Trimester } \\
2\end{array}$ \\
\hline $\mathrm{O}_{3}$ & 0,226 & & & & & & & & & & & & & & \\
\hline $\begin{array}{l}\mathrm{O}_{3} \\
\text { Trimester } 2 \\
\mathrm{O}_{3}\end{array}$ & 0,710 & $-0,090$ & & & & & & & & & & & & & \\
\hline Trimester 3 & 0,262 & $-0,627$ & $-0,065$ & & & & & & & & & & & & \\
\hline $\begin{array}{l}\mathrm{PM}_{10} \text { Entire } \\
\mathrm{PM}_{10}\end{array}$ & $-0,049$ & $-0,401$ & $-0,173$ & 0,511 & & & & & & & & & & & \\
\hline $\begin{array}{l}\text { Trimester } 1 \\
\mathrm{PM}_{10}\end{array}$ & 0,634 & $-0,281$ & 0,547 & 0,489 & 0,407 & & & & & & & & & & \\
\hline Trimester 2 & $-0,320$ & $-0,694$ & $-0,241$ & 0,545 & 0,774 & 0,121 & & & & & & & & & \\
\hline Trimester 3 & $-0,411$ & 0,359 & $-0,588$ & $-0,257$ & 0,352 & $-0,521$ & 0,076 & & & & & & & & \\
\hline $\begin{array}{l}\mathrm{NO}_{2} \text { Entire } \\
\mathrm{NO}_{2}\end{array}$ & 0,040 & $-0,326$ & $-0,111$ & 0,482 & 0,919 & 0,459 & 0,681 & 0,265 & & & & & & & \\
\hline $\begin{array}{l}\text { Trimester } 1 \\
\mathrm{NO}_{2}\end{array}$ & 0,594 & $-0,183$ & 0,472 & 0,418 & 0,375 & 0,941 & 0,041 & $-0,431$ & 0,515 & & & & & & \\
\hline $\begin{array}{l}\text { Trimester } 2 \\
\mathrm{NO}_{2}\end{array}$ & $-0,260$ & $-0,649$ & $-0,146$ & 0,476 & 0,692 & 0,154 & 0,942 & $-0,026$ & 0,690 & 0,086 & & & & & \\
\hline Trimester 3 & $-0,338$ & 0,359 & $-0,568$ & $-0,190$ & 0,347 & $-0,486$ & 0,077 & 0,955 & 0,323 & $-0,391$ & $-0,022$ & & & & \\
\hline $\begin{array}{l}\text { Temp Entire } \\
\text { temp }\end{array}$ & 0,530 & 0,549 & 0,498 & $-0,408$ & $-0,699$ & $-0,018$ & $-0,855$ & $-0,210$ & $-0,632$ & 0,034 & $-0,800$ & $-0,231$ & & & \\
\hline $\begin{array}{l}\text { Trimester } 1 \\
\text { temp }\end{array}$ & $-0,477$ & 0,638 & $-0,601$ & $-0,599$ & $-0,286$ & $-0,836$ & $-0,269$ & 0,685 & $-0,320$ & $-0,746$ & $-0,314$ & 0,661 & 0,106 & & \\
\hline $\begin{array}{l}\text { Trimester } 2 \\
\text { temp }\end{array}$ & 0,506 & 0,504 & 0,662 & $-0,557$ & $-0,600$ & 0,036 & $-0,789$ & $-0,178$ & $-0,556$ & 0,057 & $-0,712$ & $-0,226$ & 0,907 & 0,012 & \\
\hline Trimester 3 & 0,573 & $-0,505$ & 0,500 & 0,683 & 0,076 & 0,765 & 0,073 & $-0,742$ & 0,143 & 0,716 & 0,104 & $-0,695$ & 0,139 & $-0,877$ & 0,028 \\
\hline
\end{tabular}




\section{REFERÊNCIAS}

Alencar GP, Silva ZP, Santos PC, Raspantini PR, Moura BLA, Almeida MF, et al. What is the impact of interventions that prevent fetal mortality on the increase of preterm live births in the State of São Paulo, Brazil? BMC Pregnancy Childbirth. 2015;15:152.

Atkinson RW, Anderson HR, Sunyer J, Ayers J, Baccini M, Vonk JM, et al. Acute effects of particulate air pollution on respiratory admission: results from APHEA 2 project. Air pollution and health: a European approach. Am J Respir Crit Care Med. 2001;164:1860-6.

Bakonyi SMC, Danni-Oliveira IM, Martins LC, Braga ALF. Air pollution and respiratory diseases among children in Brazil. Rev Saude Publica. 2004;38(5):15.

Barbieri MA, Silva AA, Bettiol H, Gomes UA. Risk factors for the increasing trend in low birth weight among live births born by vaginal delivery, Brazil. Rev Saude Publica. 2000;34(6):596-602.

Barceló MA, Saez M, Saurina C. Spatial variability in mortality inequalities, socioeconomic deprivation, and air pollution in small areas of the Barcelona Metropolitan Region, Spain. Sci Total Environ. 2009;407:5501-23.

Blanco-Becerra LC, Miranda-Soberanis V, Barraza-Villareal A, Junger W, Hurtado-Díaz M, Romieu I. Effect of socioeconomic status on the association between air pollution and mortality in Bogota, Colombia. Salud Publica Mex. 2014;56(4):371-8.

Braga ALF, Conceição GMS, Pereira LAA, Kishi HS, Pereira JCR, Andrade MF, et al. Air pollution and pediatric respiratory hospital admissions in São Paulo, Brazil. J Environ Med. 1999;1:95-102. 
Braga ALF, Pereira LAA, Procópio M, André PA, Saldiva PHN. Associação entre poluição atmosférica e doenças respiratórias e cardiovasculares na cidade de Itabira, Minas Gerais, Brasil. Cad Saude Publica. 2007;23(4):5570-8.

Brasil. Lei n. 6.015 de 31 de dezembro de 1973. Dispõe sobre os registros públicos, e dá outras providências [Internet]. [citado 28 abr. 2020]. Disponível em: http://www.planalto.gov.br/ccivil_03/LEIS/L6015original.htm

Brasil. Ministério da Saúde. Secretaria de Vigilância em Saúde. Portaria n. 116, de 11 de fevereiro de 2009. Regulamenta a coleta de dados, fluxo e periodicidade de envio das informações sobre óbitos e nascidos vivos para o Sistema de Informações em Saúde sob gestão da Secretaria de Vigilância em Saúde. Diário Oficial da União, Brasília (DF). 200912 fev;(30):37.

Brasil. Ministério da Saúde. Secretaria de Vigilância em Saúde. Departamento de Análise de Situação de Saúde. Manual de instruções para o preenchimento da declaração de óbito. 4a ed. Brasília: Ministério da Saúde; 2011 (Série A. Normas e manuais técnicos).

Bravo MA, Son J, Freitas CU, Gouveia N, Bell ML. Air pollution and mortality in São Paulo, Brazil: effects of multiple pollutants and analysis of susceptible populations. J Expo Sci Environ Epidemiol. 2016;26:150-61.

Brook RD, Jerrett M, Brook JR, Bard RL, Finkelstein MM. The relationship between diabetes mellitus and traffic-related air pollution. J Occup Environ Med. 2008;50:32-8.

Brunt H, Barnes J, Jones SJ, Longhurst JWS, Scally G, Hayes E. Air pollution, deprivation and health: understanding relationships to add value to local air quality management policy and practice in Wales, UK. $J$ Public Health (Oxf). 2016;39(3):485-97.

Cakmak S, Dales RE, Rubio MA, Vidal CB. The risk of dying on days of higher air pollution among the socially disadvantaged elderly. Environ Res. 2011;111:388-93. 
Carbajal-Arroyo L, Miranda-Soberanis V, Medina-Ramón M, Rojas-Bracho L, Tzintzun G, Solís-Gutiérrez $\mathrm{P}$, et al. Effect of $\mathrm{PM}_{10}$ and $\mathrm{O}_{3}$ on infant mortality among residents in the Mexico City Metropolitan Area: a case-crossover analysis, 1997-2005. J Epidemiol Community Health. 2011;65(8):715-21.

Carey IM, Atkinson RW, Kent AJ, Van Staa T, Cook DG, Anderson HR. Mortality associations with long-term exposure to outdoor air pollution in a national english cohort. Am J Respir Crit Care Med. 2013;187(11):1226-33.

Caudri D, Wijga A, Gehrign U, Smit HA, Brunekreef B, Kerkhof $M$, et al. Respiratory symptoms in the first 7 years of life and birth weight at term: the PIAMA Birth Cohort. Am J Respir Crit Care Med. 2007;175(10):1078-85.

Cesaroni G, Badaloni C, Gariazzo C, Stafoggia M, Sozzi R, Davoli M, et al. Longterm exposure to urban air pollution and mortality in a cohort of more than a million adults in Rome. Environ Health Perspect. 2013;121(3):324-31.

CETESB - Companhia Ambiental do Estado de São Paulo. Qualar - Sistema de informações da qualidade do ar [Internet]. São Paulo: CETESB; [2016] [citado 25 set. 2017]. Disponível em: http://qualar.cetesb.sp.gov.br/qualar/home.do

CETESB - Companhia Ambiental do Estado de São Paulo. Qualidade do ar no estado de São Paulo: 2014. São Paulo: CETESB; 2015. (Série Relatórios).

CETESB - Companhia Ambiental do Estado de São Paulo. Qualidade do ar no estado de São Paulo: 2017. São Paulo: CETESB; 2018. (Série Relatórios)

CFM - Conselho Federal de Medicina. Resolução CFM n. 1931/2009. Aprova o Código de Ética Médica [Internet]. [citado 28 abr. 2020]. Disponível em: http://portal.cfm.org.br/index.php?option=com_content\&id=20670:resolucaocfm-no-19312009-

Chen R, Kan H, Chen B, Huang W, Bai Z, Song G, et al. Association of particulate air pollution with daily mortality: the China air pollution and health effects study. Am J Epidemiol. 2012;175(11):1173-81. 
Chernausek SD. Update: consequences of abnormal fetal growth. J Clin Endocrinol Metab. 2012;97(3):689-95.

Chi GC, Hajat A, Bird CE, Cullen MR, Griffin BA, Miller KA, et al. Individual and neighborhood socioeconomic status and the association between air pollution and cardiovascular disease. Environ Health Perspect. 2016;124(12):1840-47.

Chiusolo M, Cadum E, Stafoggia M, Galassi C, Berti G, Faustini A, et al. Shortterm effects of nitrogen dioxide on mortality and susceptibility factors in 10 italian cities: the EpiAir Study. Environ Health Perspect. 2011;119(9):1233-8.

Choi G, Heo S, Lee JT. Assessment of environmental injustice in Korea using synthetic air quality index and multiple indicators of socioeconomic status: a cross-sectional study. J Air Waste Manag Assoc. 2016;66(1):28-37.

Cohen AJ, Anderson HR, Ostro B, Pandey KD, Krzyzanowski M, Künzli N, et al. The global burden of disease due to outdoor air pollution. J Toxicol Environ Health A. 2005;68(13-14):1301-7.

CONAMA - Conselho Nacional do Meio Ambiente. Resolução CONAMA n. 3, de 28 de junho de 1990 [Internet]. [citado 28 abr. 2020]. Disponível em: http://www2.mma.gov.br/port/conama/res/res90/res0390.html

CREMERS - Conselho Regional de Medicina do Estado do Rio Grande do Sul. Manual do atestado de óbito. 2a ed. Porto Alegre: CREMERS; 2018.

Dadvand P, Parker J, Bell ML, Bonzini M, Brauer M, Darrow LA, et al. Maternal exposure to particulate air pollution and term birth weight: a multi-country evaluation of effect and heterogeneity. Environ Health Perspect. 2013:121(3); 367-373.

Deguen S, Petit C, Delbarre A, Kihal W, Padilla C, Benmarhnia T, et al. Neighbourhood characteristics and long-term air pollution levels modify the association between the short-term nitrogen dioxide concentrations and all-cause mortality in Paris. PloS One. 2015;10(7):[14 p.] 
Deguen S, Zmirou-Navier D. Social inequalities resulting from health risks related to ambient air quality - an european review. Eur J Public Health. 2010;20(1):2735.

Delfino RJ, Staimer N, Tjoa T, Arhami M, Polodori A, Gillen DL, et al. Associations of primary and secondary organic aerosols with airway and systemic inflammation in an elderly panel cohort. Epidemiology. 2010;21(6):892-902.

Dong GH, Zhang P, Sun B, Zhang L, Chen X, Ma N, et al. Long-term exposure to ambient air pollution and respiratory disease mortality in Shenyang, China: a 12-year population-based retrospective cohort study. Respiration. 2012;84:3608.

Eftim SE, Samet JM, Janes H, McDermott A, Dominici F. Fine particulate matter and mortaliy: a comparison of the six cities and American Cancer Society cohorts with a medicare cohort. Epidemiology. 2008;19(2); 209-16.

Eitan O, Yuval, Barchana M, Dubnov J, Linn S, Carmel Y, et al. Spatial analysis of air pollution and cancer incidence rates in Haifa Bay, Israel. Sci Total Environ. 2010;408:4429-39.

Erickson AC, Ostry A, Chan LHM, Arbour L. The reduction of birth weight by fine particulate matter and its modification by maternal and neighbourhood-level factors: a multilevel analysis in British Columbia, Canada. Environ Health. 2016;15:51.

Faustini A, Stafoggia M, Renzi M, Cesaroni G, Alessandrini E, Davoli M, et al. Does chronic exposure to high levels of nitrogen dioxide exacerbate the shortterm effects of airbone particles? Occup Environ Med. 2016;73:772-8.

Fecht D, Fischer P, Fortunato L, Hoek G, Hoogh K, Marra M, et al. Associations between air pollution and socioeconomic characteristics, ethnicity and age profile of neighborhoods in England and the Netherlands. Environ Pollut. 2015;198:20110. 
Ferreira TM, Forti MC, Freitas CU, Nascimento FP, Junger WL, Gouveia N. Effects of particulate matter and its chemicals constituents on elderly hospital admissions due to circulatory and respiratory diseases. Int J Environ Res Public Health. 2016;13:[11p.]

FUNASA - Fundação Nacional de Saúde. Manual de procedimentos do Sistema de Informações sobre Nascidos Vivos. Brasília: Ministério da Saúde, FUNASA; 2001.

Ghosh JKC, Heck JE, Cockburn M, Su J, Jerrett M, Ritz B. Prenatal exposure to traffic-related air pollution and risk of early childhood cancers. Am J Epidemiol. 2013;178(8):1233-9.

Gouveia N, Bremmer SA, Novaes HMD. Association between ambient air pollution and birth weight in São Paulo, Brazil. J Epidemiol Community Health. 2004;58:11-17.

Gouveia N, Fletcher T. Time series analysis of air pollution and mortality; effects by cause, age and socioeconomic status. $J$ Epidemiol Community Health. 2000;54:750-5.

Gray SC, Edwards SE, Schultz BD, Miranda ML. Assessing the impact of race, social factors and air pollution on birth outcomes: a population-based study. Environ Health. 2014;13:4.

Hales S, Blakely T, Woodward A. Air pollution and mortality in New Zealand: cohort study. J Epidemiol Community Health. 2012;66:468-73.

Henriques LB, Alves EB, Vieira FMSB, Cardoso BB, D’Angeles ACR, Cruz OG, et al. Acurácia da determinação da idade gestacional no Sistema de Informações sobre Nascidos Vivos (SINASC): um estudo de base populacional. Cad Saude Publica [Internet]. 2019 [citado abr. 2020];35(3):e00098918. Disponível em: http://www.scielo.br/scielo.php?script=sci_arttext\&pid=S0102-

$311 \times 2019000305011$ 
Honda T, Pun VC, Manjourides J, Suh H. Anemia prevalence and hemoglobin levels are associated with long-term exposure to air pollution in an older population. Environ Int. 2017;101:125-32.

IBGE - Instituto Brasileiro de Geografia e Estatística [Internet]. Rio de Janeiro: IBGE - Instituto Brasileiro de Geografia e Estatística; c2017 [citado 10 mai. 2018]. São Paulo [municipality]: panorama. Disponível em: https://cidades.ibge.gov.br/brasil/sp/sao-paulo/panorama

Jerrett M, Burnett RT, Pope CA 3rd, Ito K, Thurston G, Krewski D, et al. Longterm ozone exposure and mortality. N Engl J Med. 2009;360(11):1085-95.

Keijzer C, Agis D, Ambrós A, Arévalo G, Baldasano JM, Bande S, et al. The association of air pollution and greenness with mortality and life expectancy in Spain: a small-area study. Environ Int. 2017;99:170-6.

Kim J, Kim H, Kweon J. Hourly diferences in air pollution on risk of asthma exacerbation. Environ Pollut. 2015;203:15-21.

Kloog I, Ridgway B, Koutrakis P, Coull BA, Schwartz JD. Long- and short-term exposure to $\mathrm{PM}_{2.5}$ and mortality: using novel exposure models. Epidemiology. 2013;24(4):555-61.

Krämer U, Herder C, Sugiri D, Strassburger K, Schikowski T, Ranft U, et al. Traffic-related air pollution and incident type 2 diabetes: results from the SALIA cohort study. Environ Health Perspect. 2010;118(9):1273-9.

Laurent O, Hu J, Li L, Cockburn M, Escobedo L, Kleeman MJ, et al. Sources of contents of air pollution affecting term low birth weight in Los Angeles County, California, 2001-2008. Environ Res. 2014;134;488-95.

Levy D, Lumley T, Sheppard L, Kaufman J, Checkoway H. Referent selection in case-crossover analysis of acute health effects of air pollution. Epidemiology. 2001;12(2):186-92 
Li L, Laurent O, Wu J. Spatial variability of the effect of air pollution on term birth weight: evaluating influential factors using a Bayesian hierarchical models. Environ Health. 2016;15:14.

Li S, Guo Y, Williams G. Acute impact of hourly ambient air pollution on preterm birth. Environ Health Perspect. 2016;124(10):1623-9.

Lin CA, Martins MA, Farhat SC, Pope CA 3rd, Conceição GMS, Anastácio MV, et al. Air pollution and respiratory illness of children in São Paulo, Brazil. Paediatr Perinat Epidemiol. 1999;13(4):475-88.

Madsen C, Rosland P, Hoff DA, Nystad W, Nafstad P, Næss ØE. The short-term effect of 24-h average and peak air pollution on mortality in Oslo, Norway. Eur $J$ Epidemiol. 2012;27:717-27.

Maheswaran R, Pearson T, Smeeton NC, Beevers SD, Campbell MJ, Wolfe CD. Impact of outdoor air pollution on survival after stroke: population-based cohort study. Stroke. 2010;41:869-77.

Martins LC, Latorre MRDO, Cardoso MRA, Gonçalves FLT, Saldiva PHN, Braga ALF. Poluição atmosférica e atendimentos por pneumonia e gripe em São Paulo, Brasil. Rev Saude Publica. 2002;36:88-94.

Mathews JM, MacDorman MF. Infant mortality statistics from 2008 period linked birth/infant death data set national. Natl Vital Stat Rep. 2012;60(5):1-27.

Mello Jorge MHP, Gotlieb SLD, Soboll MLMS, Almeida MF, Latorre MRDO. Avaliação do sistema de informação sobre nascidos vivos e o uso de seus dados em epidemiologia e estatísticas de saúde. Rev Saude Publica. 1993;27(Supl.):146.

Moore JX, Akinyemiju T, Wang HE. Pollution and regional variations of lung cancer mortality in the United States. Cancer Epidemiol. 2017;49:118-27.

Morello-Frosch R, Jesdale BM, Sadd JL, Pastor M. Ambient air pollution and fullterm birth weight in California. Environ Health. 2010;9:44.d 
O'Lenick CR, Winquist A, Mulholland JA, Friberg MD, Chang $\mathrm{HH}$, Kramer MR, et al. Assessment of neighbourhood-level socioeconomic status as a modifier of air pollution-asthma associations among children in Atlanta. J Epidemiol Community Health. 2017;71:129-36.

OMS - Organização Mundial da Saúde. Classificação estatística internacional de doenças e problemas relacionados à saúde. 10a revisão. São Paulo: Centro da OMS para a Classificação de Doenças em Português; 1995.

O'Neill MS, Bell ML, Ranjit N, Cifuentes LA, Loomis D, Gouveia N, et al. Air pollution and mortality in Latin America: the role of education. Epidemiology. 2008;19(6):810-9.

O’Neill MS, Jerett M, Kawachi I, Levy JI, Cohen AJ, Gouveia N, et al. Health, wealth, and air pollution: advancing theories and methods. Environ Health Perspect. 2003;111(16):1861-70.

Ou CQ, Hedley AJ, Chung RY, Thach TQ, Chau YK, Chan KP, et al. Socioeconomic disparities in air pollution-associated mortality. Environ Res. 2008;107:237-44.

Padula AM, Mortimer KM, Tager IB, Hammond K, Lurmann FW, Yang W, et al. Traffic-related air pollution and risk of preterm birth in the San Joaquin Valley of California. Ann Epidemiol. 2014;24(12):888-95.

Pinault L, Crouse D, Jerrett M, Brauer M, Tjepkema M. Socioeconomic differences in nitrogen dioxide ambient air pollution exposure among children in the three largest Canadian cities. Health Rep. 2016;27(7):3-9.

Pinault L, Van Donkelaar A, Martin RV. Exposure to fine particulate matter air pollution in Canada. Health Rep. 2017a;28(3):9-16.

Pinault LL, Weichenthal S, Crouse DL, Brauer M, Erickson A, Van Donkelaar A, et al. Associations between fine particulate matter and mortality in the 2001 
Canadian Census Health and Environment Cohort. Environ Res. 2017b;159:40615.

Qiu H, Tian L, Ho KF, Pun VC, Wang X, Yu ITS. Air pollution and mortality: effect modification by personal characteristics and specific cause of death in a caseonly study. Environ Pollut. 2015;199:192-7.

Ren C, Melly S, Schwartz J. Modifiers of short-term effects of ozone on mortality in eastern Massachusetts: a case-crossover analysis at individual level. Environ Health. 2010;9:3.

Rich DQ, Demissie K, Lu SE, Kamat L, Wartenberg D, Rhoads GG. Ambient air pollutant concentrations during pregnancy and the risk of fetal growth restriction. J Epidemiol Community Health. 2009;63:488-96.

Richardson EA, Pearce J, Kingham S. Is particulate air pollution associated with health and health inequalities in New Zealand? Health Place. 2011;17:1137-43.

Romão R, Pereira LAA, Saldiva PHN, Pinheiro PM, Braga ALF, Martins LC. The relationship between low birth weight and exposure to inhalable particulate matter. Cad Saude Publica. 2013;29(6):1101-08.

Rosenlund M, Bellander T, Nordquist T, Alfredsson L. Traffic-generated air pollution and myocardial infarction. Epidemiology. 2009;20(2):265-71.

São Paulo (cidade). São Paulo: Prefeitura Municipal de São Paulo; c2017 [citado 28 abr. 2020]. PRO-AIM - Programa de Aprimoramento das Informações de Mortalidade. Disponível em: https://www.prefeitura.sp.gov.br/cidade/secretarias/saude/epidemiologia_e_info rmacao/mortalidade/index.php? $\mathrm{p}=29586$

Schifano P, Lallo A, Asta F, De Sario M, Davoli M, Michelozzi P. Effect of ambient temperature and air pollutants on the risk of preterm birth, Rome 2001-2010. Environ Int. 2013;61:77-87.

Schwartz J, Dockery DW. Particulate air pollution and daily mortality in Steubenville, Ohio. Am J Epidemiol. 1992;135:12-9. 
Šrám JR, Binková B, Dejmek J, Bobak M. Ambient air pollution and pregnancy outcomes: a review of the literature. Environ Health Perspect. 2005:113;375-82.

Stieb DM, Chen L, Beckerman BS, Jerret M, Crouse DL, Omariba DWR, et al. Associations of pregnancy outcomes and $\mathrm{PM}_{2.5}$ in a National Canadian Study. Environ Health Perspect. 2016;124(2):243-9.

Stieb DM, Chen L, Eshoul M, Judek S. Ambient air pollution, birth weight and preterm birth: a systematic review and meta-analysis. Environ Res. 2012:117;100-111.

Takiya H. Atlas Ambiental do Município de São Paulo: fase l: diagnóstico e bases para a definição de políticas públicas para as áreas verdes no município de São Paulo. São Paulo: Prefeitura do Município de São Paulo. Secretaria Municipal do Meio Ambiente, Secretaria Municipal de Planejamento Urbano; Jul. 2002. Relatório final. Processo no: 1999/10955-9. Projeto Biota - FAPESP.

Vinson F, Merhi M, Baldi I, et al. Exposure to pesticides and risk of childhood cancer: a meta-analysis of recent epidemiological studies. Occup Environ Med. 2011;68(9):694-702.

Wang Y, Shi L, Lee M, Liu P, Di Q, Zanobetti A, et al. Long-term exposure to $\mathrm{PM}_{2.5}$ and mortality among older adults in the Southeastern US. Epidemiology. 2017;28(2):207-14.

WHO - World Health Organization. Global nutrition targets 2025: low birth weight policy brief [Internet]. Geneva: WHO; 2014 [citado 22 out. 2019]. Disponível em: http://apps.who.int/iris/bitstream/10665/149020/2/WHO_NMH_NHD_14.5_eng.p df?ua=1

WHO - World Health Organization. Training for the Health Sector. Outdoor air pollution [slides em pdf]. Geneva: WHO; 2008 [citado 14 out. 2017]. Disponível em: https://www.who.int/ceh/capacity/Outdoor_air_pollution.pdf 
Wong CM, Ou CQ, Chan KP, Chau YK, Thach TQ, Yang L, et al. The effects of air pollution on mortality in socially deprived urban areas in Hong Kong, China. Environ Health Perspect. 2008;116(9):1189-94.

Wu J, Ren C, Delfino RJ, Chung J, Wilhelm M, Ritz B. Association between local traffic-generated air pollution and preeclampsia and preterm delivery in the south coast air basin of California. Environ Health Perspect. 2009;177(11):1773-79.

Yang Y, Tang R, Qiu H, Lai PC, Wong P, Thach TQ, et al. Long term exposure to air pollution and mortality in an elderly cohort in Hong Kong. Environ Int. 2018;117:99-106.

Yap P, Gilbreath S, Garcia C, Jareen N, Goodrich B. The Influence of socioeconomic markers on the association between fine particulate matter and hospital admissions for respiratory conditions among children. Am J Public Health. 2013;103(4):695-702.

Yi O, Kim H, Ha E. Does area level socioeconomic status modify the effects of $\mathrm{PM}_{10}$ on preterm delivery? Environ Res. 2010;110:55-61.

Yorifuji T, Naruse H, Kashima S, Takao S, Murakoshi T, Doi H, et al. Residential proximity to major roads and adverse birth outcomes: a hospital-based study. Environ Health. 2013;12:34.

Zhang LW, Chen X, Xue XD, Sun M, Han B, Li CP, et al. Long-term exposure to high particulate matter pollution and cardiovascular mortality: a 12-year cohort study in four cities in northern China. Environ Int. 2014;62:41-7.

Zhang P, Dong G, Sun B, Zhang L, Chen X, Ma N, et al. Long-term exposure to ambient air pollution and mortality due to cardiovascular disease and cerebrovascular disease in Shenyang, China. PLoS One. 2011;6(6):[8 p.]. 


\section{BIBLIOGRAFIA CONSULTADA}

Arbex MA, Conceição GMS, Cendon SP, Arbex FF, Lopes AC, Moysés EP, et al. Urban air pollution and chronic obstructive pulmonary disease-related emergency department visits. J Epidemiol Community Health. 2009;63:777-783.

Auger N, Fraser WD, Smargiassi A, Bilodeau-Bertrand M, Kosatsky T. Elevated outdoor temperatures and risk of stillbirth. Int J Epidemiol. 2017;46(1):200-8.

Buriol VCS, Hirakata V, Goldani MZ, Silva CH. Temporal evolution of the risk factors associated with low birth weight rates in Brazilian capitals (1996-2011). Popul Health Metr. 2016;14:15.

Cohen B. Urban growth in developing countries: a review of current trends and a caution regarding existing forecasts. World Development. 2004;32(1):23-51.

Finkelstein MM, Jerrett M, Sears MR. Environmental inequality and circulatory disease mortality gradients. J Epidemiol Community Health. 2005;59(6):481-7.

Forastiere F, Stafoggia M, Tasco C, Picciotto S, Agabiti N, Cesarion G, et al. Socioeconomic status, particulate air pollution, and daily mortality: differential exposure or differential susceptibility. Am J Ind Med. 2007;50(3):208-16.

Junger WL. Análise, imputação de dados e interfaces computacionais em estudos de séries temporais epidemiológicas [tese]. Rio de Janeiro: Centro de Biomédico, Universidade do Estado do Rio de Janeiro; 2008.

Laurent O, Bard D, Filleul L, Segala C. Effect of socioeconomic status on the relationship between atmospheric pollution and mortality. $J$ Epidemiol Community Health. 2007;61:665-75. 
Li C, Balluz LS, Vaidyanathan A, Wen XJ, Hao Y, Qualters JR. Long-term exposure to ozone and life expectancy in the United States, 2002 to 2008. Medicine (Baltimore). 2016;95(7):1-8.

Martins MC, Fatigati FL, Vespoli TC, Martins LC, Pereira LA, Martins MA, et al. Influence of socioeconomic conditions on air pollution adverse health effects in elderly people: an analysis of six regions in São Paulo, Brazil. J Epidemiol Community Health. 2004;58(1):41-6.

Neidell MJ. Air pollution, health, and socio-economic status: the effect of outdoor air quality on childhood asthma. J Health Econ. 2004;23(6):1209-36.

Pereira LA Loomis D, Conceição GM, Braga AL, Arcas RM, Kishi HS, et al. Association between air pollution and intrauterine mortality in São Paulo, Brazil. Environ Health Perspect. 1998;106(6):325-29.

Ritz B, Yu F, Fruin S, Chapa G, Shaw GM, Harris JA. Ambient air pollution and risk of birth defects in southern California. Am J Epidemiol. 2002;155(1):17-25.

Vinson F, Merhi M, Baldi I, Raynal H, Gamet-Payrastre L. Exposure to pesticides and risk of childhood cancer: a meta-analysis of recent epidemiological studies. Occup Environ Med. 2011;68(9):694-702. 


\section{APÊNDICE}

\section{MODELO DA DECLARAÇÃO DE NASCIDO VIVO}

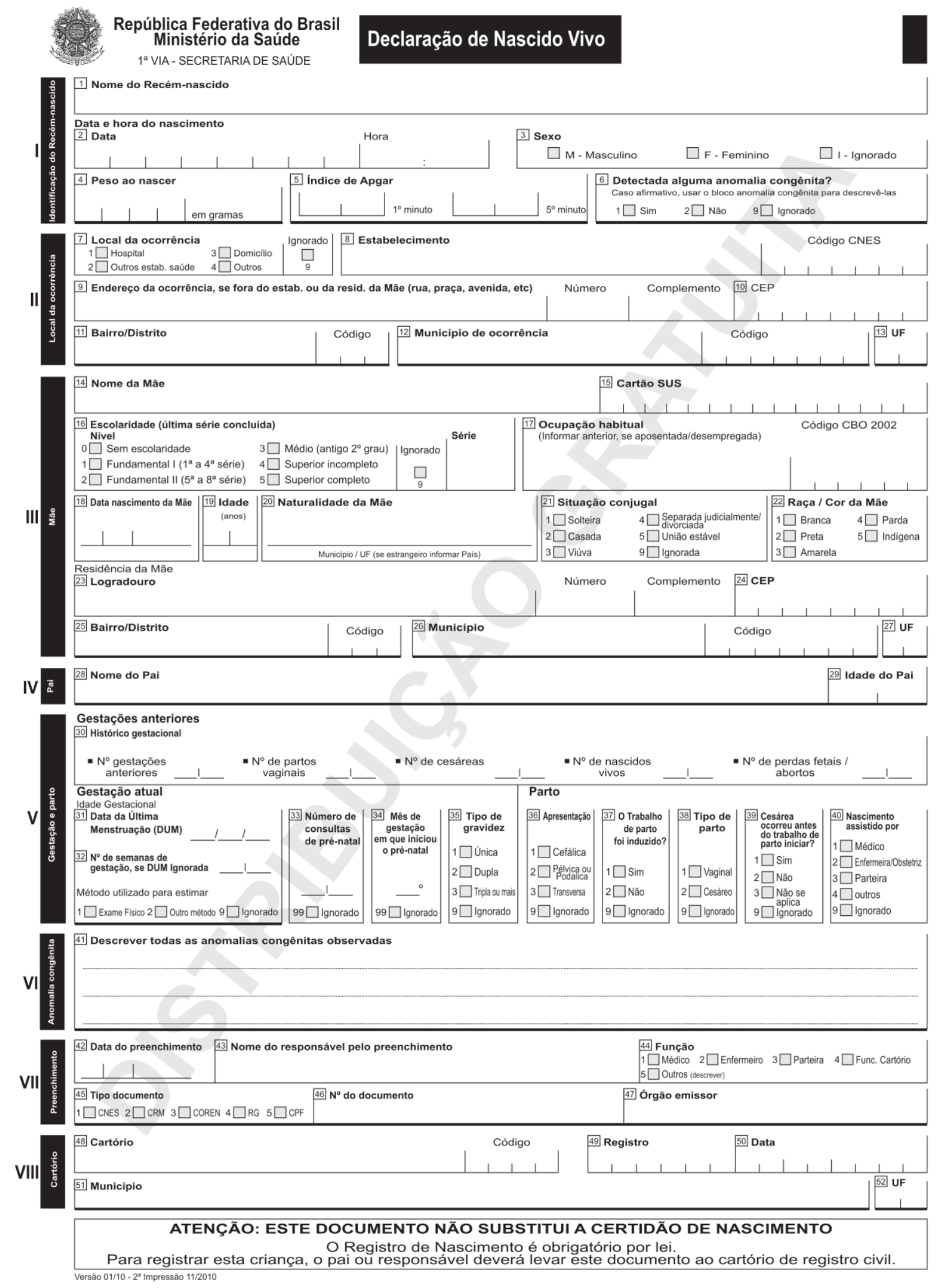




\section{MODELO DA DECLARAÇÃO DE ÓBITO}

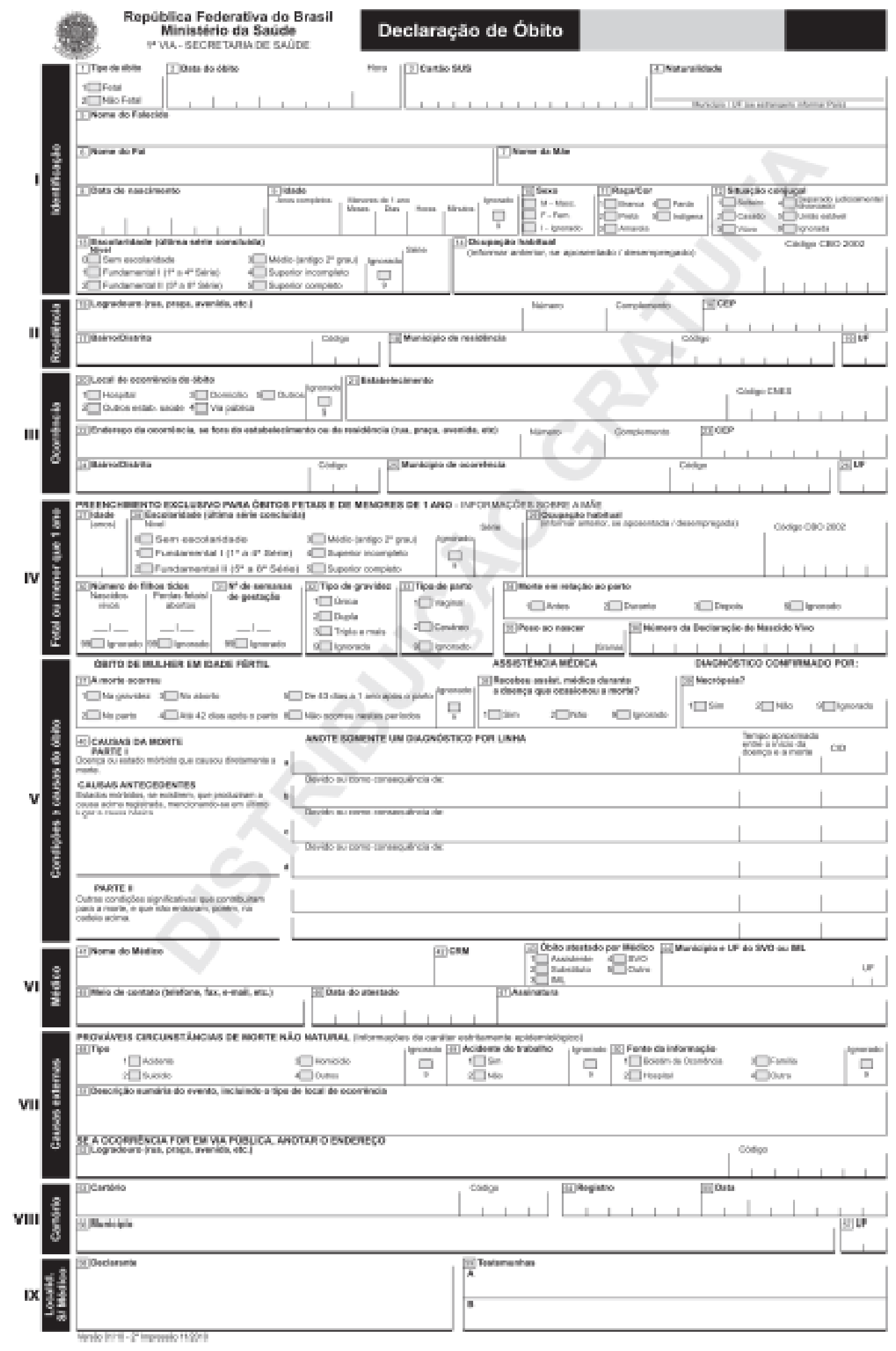




\title{
Aprovação no comitê de ética e pesquisa
}

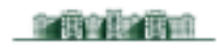 \\ MEDICINA \\ TSP \\ COMITÊ DE ÉTICA EM PESQUISA
}

\begin{abstract}
APROVAÇÃO
- Comitê de Ética em Pesquisa da Faculdade de Medicina da Universidade de São Paulo, aprovou 22/11/2017, o Protocolo de Pesquisa $n^{\circ} 3^{85 / 17}$ intitulado: A condição socioeconomica alterada os efeitos da poluição do ar na saúde? apresentado pelo Departamento Medicina Preventiva.

Cabe ao pesquisador elaborar e apresentar ao CEP-FMUSP, os relatórios parciais e final sobre a pesquisa (Resolução do Conselho Nacional de Saúde no $466 / 12$, inciso $\mathrm{IX}_{2}$, letra " $"$ ").
\end{abstract}

Pesquisador (a) Responsável: Nelson da Cruz Gouveia

Pesquisador (a) Executante: Felipe Parra do Nascimento

CEP-FMUSP, 22 de Novembro de 2017.

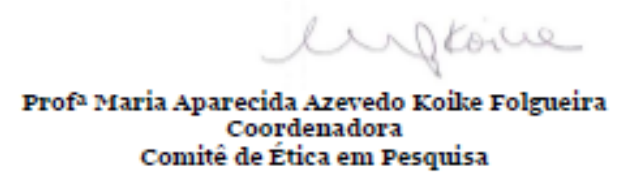

Comitê de Ética em Pesquisa 


\section{Índice de Apgar}

O índice de Apgar, criado em 1949 pela anestesista inglesa Virgínia Apgar, é uma maneira de avaliar as condições do recém-nascido.

O índice de Apgar de cada recém-nascido e consta em sua respectiva declaração de Nascido Vivo. O índice consiste na avaliação de 5 sinais objetivos do recém-nascido no primeiro e no quinto minuto após o nascimento. Os sinais avaliados são: frequencia cardíaca, esforço respiratório, tônus muscular, cor da pele e irritabilidade reflexa. Para cada um desses sinais é atribuída uma nota entre 0 e 2, sendo o índice a soma das 5 notas.

- Frequencia cardíaca: verifica os batimentos cardíacos do recém-nascido - 0 se ausente, 1 se $<100$ bpm, 2 se $>100 \mathrm{bpm}$.

- Esforço respiratório: verifica se o choro está forte, fraco ou ausente - 0 se não há choro, 1 se choro fraco, 2 se choro forte.

- Tônus muscular: observa se o bebê tem boa flexão dos membros ou se está flácido - 0 se flácido, 1 se flexão de pernas e braços, 2 se movimento ativo.

- Cor da pele: observa se a pele está rosada ou azulada, total ou parcialmente - 0 se cianótico ou pálido, 1 se cianose nas extremidades apenas, 2 se rosado.

- Irritabilidade reflexa: verifica se o bebê reage aos estímulos - 0 se ausente, 1 com algum movimento, 2 espirros ou choro.

Após a soma das notas, que variam entre 0 e 10, resulta o índice de Apgar do recém-nascido, que é classificado da seguinte forma:

Sem asfixia (Apgar entre 8 e 10)

Com asfixia leve (Apgar entre 5 e 7)

Com asfixia moderada (Apgar entre 3 e 4)

Com asfixia grave (Apgar entre 0 e 2 ) 